Pacific

Journal of

Mathematics

\title{
THE FIXED POINT SUBALGEBRA OF A LATTICE VERTEX OPERATOR ALGEBRA BY AN AUTOMORPHISM OF ORDER THREE
}

Kenichiro TANABE AND HiROMICHI YAMADA 


\title{
THE FIXED POINT SUBALGEBRA OF A LATTICE VERTEX OPERATOR ALGEBRA BY AN AUTOMORPHISM OF ORDER THREE
}

\author{
KENICHIRO TANABE AND HiROMICHI YAMADA
}

\begin{abstract}
We study the subalgebra of the lattice vertex operator algebra $V_{\sqrt{2} A_{2}}$ consisting of the fixed points of an automorphism which is induced from an order-three isometry of the root lattice $A_{2}$. We classify the simple modules for the subalgebra. The rationality and the $C_{2}$-cofiniteness are also established.
\end{abstract}

\section{Introduction}

The space of fixed points of an automorphism group of finite order in a vertex operator algebra is a vertex operator subalgebra. The study of such subalgebras and their modules is called orbifold theory. It is a rich field both in conformal field theory and in the theory of vertex operator algebras. However, orbifold theory is difficult to study in general. One reason is that the subalgebra of fixed points usually has more complicated structure than the original vertex operator algebra.

The first example of orbifold theory in vertex operator algebras is the moonshine module $V^{\natural}$ by Frenkel, Lepowsky, and Meurman [Frenkel et al. 1988], constructed as an extension of $V_{\Lambda}^{+}$by its simple module $V_{\Lambda}^{T,+}$, where $V_{\Lambda}^{+}$is the space of fixed points of an automorphism $\theta$ of order two in the Leech lattice vertex operator algebra $V_{\Lambda}$. This construction is called a $2 B$-orbifold construction because $\theta$ corresponds to a $2 B$ involution of the monster simple group. More generally, Frenkel et al. defined a vertex operator algebra $V_{L}$ associated with an arbitrary positive definite even lattice $L$. These lattice vertex operator algebras provide a large family of vertex operator algebras. Such a lattice vertex operator algebra admits an automorphism $\theta$ of order two, which is a lift of the isometry $\alpha \mapsto-\alpha$ of the underlying lattice $L$. Orbifold theory for the fixed point subalgebra $V_{L}^{+}$of $\theta$ has been developed extensively. The simple $V_{L}^{+}$-modules have been classified

MSC2000: primary 17B69; secondary 17B68.

Keywords: vertex operator algebra, orbifold, $W_{3}$ algebra.

Tanabe was partially supported by JSPS Grant-in-Aid for Scientific Research No. 17740002. Yamada was partially supported by JSPS Grant-in-Aid for Scientific Research No. 17540016. 
[Abe and Dong 2004], the fusion rules have been determined [Abe et al. 2005], and it has been established that $V_{L}^{+}$is $C_{2}$-cofinite [Abe et al. 2004; Yamskulna 2004].

Here we study the fixed point subalgebra by an automorphism of order three for a certain lattice vertex operator algebra. Namely, let $L=\sqrt{2} A_{2}$ be $\sqrt{2}$ times an ordinary root lattice of type $A_{2}$ and let $\tau$ be an isometry of the root lattice of type $A_{2}$ induced from an order-three permutation on the set of positive roots. We classify the simple modules for the subalgebra $V_{L}^{\tau}$ of fixed points by $\tau$. Moreover, we show that $V_{L}^{\tau}$ is rational and $C_{2}$-cofinite.

In [Dong et al. 2004; Kitazume et al. 2003] we have already discussed the vertex operator algebra $V_{L}^{\tau}$. It was shown that $V_{L}^{\tau}=M^{0} \oplus W^{0}$ is a direct sum of a subalgebra $M^{0}$ and its simple highest-weight module $W^{0}$. Actually, $M^{0}$ is a tensor product of a $W_{3}$ algebra of central charge $6 / 5$ and a $W_{3}$ algebra of central charge $4 / 5$. The property of a $W_{3}$ algebra of central charge $6 / 5$ as the first component of the tensor product $M^{0}$ was investigated in [Dong et al. 2004]. It is generated by the Virasoro element $\tilde{\omega}^{1}$ and a weight-three vector $J$. The second component of $M^{0}$, a $W_{3}$ algebra of central charge 4/5, was studied in [Kitazume et al. 2000b]. It is generated by the Virasoro element $\tilde{\omega}^{2}$ and a weight-three vector $K$. Each of these $W_{3}$ algebras possesses a symmetry of order three. The order-three symmetry of the second $W_{3}$ algebra is related to the $\mathbb{Z}_{3}$ part of $L^{\perp} / L \cong \mathbb{Z}_{2} \times \mathbb{Z}_{2} \times \mathbb{Z}_{3}$, where $L^{\perp}$ denotes the dual lattice of $L$. As an $M^{0}$-module, $W^{0}$ is generated by a highestweight vector $P$ of weight 2 . Thus the vertex operator algebra $V_{L}^{\tau}$ is generated by the five elements $\tilde{\omega}^{1}, \tilde{\omega}^{2}, J, K$, and $P$.

There are 12 inequivalent simple $V_{L}$-modules, which correspond to the cosets of $L$ in its dual lattice $L^{\perp}$ [Dong 1993]. Let $\left(U, Y_{U}\right)$ be a simple $V_{L}$-module. One can define a new simple $V_{L}$-module $\left(U \circ \tau, Y_{U \circ \tau}\right)$ by $U \circ \tau=U$ as vector spaces and $Y_{U \circ \tau}(v, z)=Y_{U}(\tau v, z)$ for $v \in V_{L}$. Then $U \mapsto U \circ \tau$ is a permutation on the set of simple $V_{L}$-modules. In the case where $U$ and $U \circ \tau$ are equivalent $V_{L}$-modules, $U$ is said to be $\tau$-stable. If $U$ is $\tau$-stable, the eigenspace $U(\varepsilon)$ of $\tau$ with eigenvalue $\xi^{\varepsilon}$, where $\xi=\exp (2 \pi \sqrt{-1} / 3), \varepsilon=0,1,2$, is a simple $V_{L}^{\tau}$-module, while if $U$ belongs to a $\tau$-orbit of length three, $U$ itself is a simple $V_{L}^{\tau}$-module and the three members in the $\tau$-orbit are equivalent [Dong and Yamskulna 2002, Theorem 6.14]. Among the 12 inequivalent simple $V_{L}$-modules, three are $\tau$-stable and the remaining nine are divided into three $\tau$-orbits. In this way we obtain 12 simple $V_{L}^{\tau}$-modules. It is known that there are three inequivalent simple $\tau$-twisted $V_{L}$-modules and three inequivalent simple $\tau^{2}$-twisted $V_{L}$-modules. We denote them respectively by

$$
V_{L}^{j}(\tau):=V_{L}^{T_{\chi_{j}}}(\tau), \quad V_{L}^{j}\left(\tau^{2}\right):=V_{L}^{T_{\chi_{j}^{\prime}}}\left(\tau^{2}\right), \quad j=0,1,2 .
$$

The automorphism $\tau$ acts on these $\tau$-twisted or $\tau^{2}$-twisted $V_{L}$-modules and each eigenspace of $\tau$ is a simple $V_{L}^{\tau}$-module [Miyamoto and Tanabe 2004, Theorem 2]. 
There are 18 such simple $V_{L}^{\tau}$-modules, all of them inequivalent. Hence there are at least 30 inequivalent simple $V_{L}^{\tau}$-modules.

The main part of our argument is to show that every simple $V_{L}^{\tau}$-module is isomorphic to one of these 30 simple $V_{L}^{\tau}$-modules. Recall that $V_{L}^{\tau}=M^{0} \oplus W^{0}$ and that $M^{0}$ is a tensor product of two $W_{3}$ algebras. The $W_{3}$ algebra of central charge $6 / 5$ (resp. 4/5) possesses 20 (resp. 6) inequivalent simple modules. Thus there are 120 inequivalent simple $M^{0}$-modules. It turns out that among these simple $M^{0}$ modules, 60 of them cannot appear as an $M^{0}$-submodule in any simple $V_{L}^{\tau}$-module and that each simple $V_{L}^{\tau}$-module is a direct sum of two of the remaining 60 simple $M^{0}$-modules. We note that $W^{0}$ is not a simple current $M^{0}$-module. Thus $V_{L}^{\tau}$ is a nonsimple current extension of $M^{0}$. A discussion on simple modules for another nonsimple current extension of a certain vertex operator algebra can be found in [Lam et al. 2005, Appendix C].

The organization of this paper is as follows. In Section 2 we review various notions about untwisted or twisted modules for vertex operator algebras, together with some basic tools which will be used in later sections. In Section 3 we fix notation for the vertex operator algebra $V_{L}^{\tau}$ and collect its properties. We clarify an argument on the simplicity of $M_{T}^{0}\left(\tau^{i}\right)$ and $W_{T}^{0}\left(\tau^{i}\right), i=1,2$, in [Kitazume et al. 2003, Proposition 6.8]. Furthermore, we correct some misprints in [Kitazume et al. 2003, (6.46)] and in an equation of [Dong et al. 2004, page 265] concerning a decomposition of a simple $\tau$-twisted $V_{L}$-module $V_{L}^{j}(\tau), j=1,2$ as a $\tau$-twisted $M_{k}^{0} \otimes M_{t}^{0}$-module (see Remark 3.5). In Section 4 we discuss the structure of the 30 known simple $V_{L}^{\tau}$-modules. In particular, we calculate the action of $o\left(\tilde{\omega}^{1}\right)$, $o\left(\tilde{\omega}^{2}\right), o(J), o(K)$, and $o(P)$ on the top level of these simple modules. Finally, in Section 5 we complete the classification of simple $V_{L}^{\tau}$-modules. We also show the rationality of $V_{L}^{\tau}$.

The authors would like to thank Ching Hung Lam, Masahiko Miyamoto, and Hiroshi Yamauchi for valuable discussions. The proof of Lemma 5.7 is essentially the same as that of [Lam et al. 2005, Lemma C.3]. Part of our calculation was done by a computer algebra system Risa/Asir. The authors are grateful to Kazuhiro Yokoyama for helpful advice on computer programs.

\section{Preliminaries}

We recall some notation for untwisted or twisted modules for a vertex operator algebra. We also review the twisted version of Zhu's theory. A basic reference to twisted modules is [Dong et al. 1998a]. For untwisted modules, see also [Lepowsky and Li 2004]. Let $(V, Y, \mathbf{1}, \omega)$ be a vertex operator algebra and $g$ be an automorphism of $V$ of finite order $T$. Set $V^{r}=\left\{v \in V \mid g v=e^{2 \pi \sqrt{-1} r / T} v\right\}$, so that $V=\bigoplus_{r \in \mathbb{Z} / T \mathbb{Z}} V^{r}$. 
Definition 2.1. A weak g-twisted $V$-module $M$ is a vector space equipped with a linear map

$$
Y_{M}(\cdot, z): v \in V \mapsto Y_{M}(v, z)=\sum_{n \in \mathbb{Q}} v_{n} z^{-n-1} \in(\text { End } M)\{z\}
$$

satisfying the following conditions.

(1) $Y_{M}(v, z)=\sum_{n \in r / T+\mathbb{Z}} v_{n} z^{-n-1}$ for $v \in V^{r}$.

(2) $v_{n} w=0$ if $n \gg 0$, where $v \in V$ and $w \in M$.

(3) $Y_{M}(\mathbf{1}, z)=\operatorname{id}_{M}$.

(4) For $u \in V^{r}$ and $v \in V$, the $g$-twisted Jacobi identity holds:

$$
\begin{array}{r}
z_{0}^{-1} \delta\left(\frac{z_{1}-z_{2}}{z_{0}}\right) Y_{M}\left(u, z_{1}\right) Y_{M}\left(v, z_{2}\right)-z_{0}^{-1} \delta\left(\frac{z_{2}-z_{1}}{-z_{0}}\right) Y_{M}\left(v, z_{2}\right) Y_{M}\left(u, z_{1}\right) \\
=z_{2}^{-1}\left(\frac{z_{1}-z_{0}}{z_{2}}\right)^{-r / T} \delta\left(\frac{z_{1}-z_{0}}{z_{2}}\right) Y_{M}\left(Y\left(u, z_{0}\right) v, z_{2}\right) .
\end{array}
$$

Compare the coefficients of $z_{0}^{-l-1} z_{1}^{-m-1} z_{2}^{-n-1}$ in both sides of (2-1) for $u \in V^{r}$, $v \in V^{s}, l \in \mathbb{Z}, m \in \frac{r}{T}+\mathbb{Z}$, and $n \in \frac{s}{T}+\mathbb{Z}$. Then we obtain

$$
\sum_{i=0}^{\infty}\left(\begin{array}{c}
m \\
i
\end{array}\right)\left(u_{l+i} v\right)_{m+n-i}=\sum_{i=0}^{\infty}(-1)^{i}\left(\begin{array}{l}
l \\
i
\end{array}\right)\left(u_{l+m-i} v_{n+i}-(-1)^{l} v_{l+n-i} u_{m+i}\right)
$$

In the case $l=0$, this reduces to

$$
\left[u_{m}, v_{n}\right]=\sum_{i=0}^{\infty}\left(\begin{array}{c}
m \\
i
\end{array}\right)\left(u_{i} v\right)_{m+n-i} .
$$

The Virasoro element $\omega$ is contained in $V^{0}$. Let $L(n)=\omega_{n+1}$ for $n \in \mathbb{Z}$. Then

$$
\begin{aligned}
{[L(m), L(n)] } & =(m-n) L(m+n)+\frac{1}{12}\left(m^{3}-m\right) \delta_{m+n, 0}(\operatorname{rank} V), \\
\frac{d}{d z} Y_{M}(v, z) & =Y_{M}(L(-1) v, z)
\end{aligned}
$$

for $v \in V$; see [Dong et al. 1998a, (3.8), (3.9)].

An important consequence of (2-1) is the associativity formula

$$
\left(z_{0}+z_{2}\right)^{k+r / T} Y_{M}\left(u, z_{0}+z_{2}\right) Y_{M}\left(v, z_{2}\right) w=\left(z_{2}+z_{0}\right)^{k+r / T} Y_{M}\left(Y\left(u, z_{0}\right) v, z_{2}\right) w
$$

(see [Dong et al. 1998a, (3.5)]), where $u \in V^{r}, v \in V, w \in M$, and $k$ is a nonnegative integer such that $z^{k+r / T} Y_{M}(u, z) w \in M \llbracket z \rrbracket$.

Let $\left(M, Y_{M}\right)$ and $\left(N, Y_{N}\right)$ be weak $g$-twisted $V$-modules. A homomorphism of $M$ to $N$ is a linear map $f: M \rightarrow N$ such that $f Y_{M}(v, z)=Y_{N}(v, z) f$ for all $v \in V$.

Let $\mathbb{N}$ be the set of nonnegative integers. 
Definition 2.2. A $\frac{1}{T} \mathbb{N}$-graded weak $g$-twisted $V$-module $M$ is a weak $g$-twisted $V$-module with a $\frac{1}{T} \mathbb{N}$-grading $M=\bigoplus_{n \in \frac{1}{T} \mathbb{N}} M_{(n)}$ such that

$$
v_{m} M_{(n)} \subset M_{(n+\mathrm{wt}(v)-m-1)}
$$

for any homogeneous vectors $v \in V$.

A $\frac{1}{T} \mathbb{N}$-graded weak $g$-twisted $V$-module here is called an admissible $g$-twisted $V$-module in [Dong et al. 1998a]. Without loss we can shift the grading of a $\frac{1}{T} \mathbb{N}$ graded weak $g$-twisted $V$-module $M$ so that $M_{(0)} \neq 0$ if $M \neq 0$. We call such an $M_{(0)}$ the top level of $M$.

Definition 2.3. A $g$-twisted $V$-module $M$ is a weak $g$-twisted $V$-module with a $\mathbb{C}$-grading $M=\bigoplus_{\lambda \in \mathbb{C}} M_{\lambda}$, where $M_{\lambda}=\{w \in M \mid L(0) w=\lambda w\}$. Moreover, each $M_{\lambda}$ is a finite dimensional space and for any fixed $\lambda, M_{\lambda+n / T}=0$ for all sufficiently small integers $n$.

A $g$-twisted $V$-module is sometimes called an ordinary $g$-twisted $V$-module. By [Dong et al. 1998a, Lemma 3.4], any $g$-twisted $V$-module is a $\frac{1}{T} \mathbb{N}$-graded weak $g$-twisted $V$-module. Indeed, assume that $M$ is a $g$-twisted $V$-module. For each $\lambda \in \mathbb{C}$ with $M_{\lambda} \neq 0$, let $\lambda_{0}=\lambda+m / T$ be such that $m \in \mathbb{Z}$ is minimal subject to $M_{\lambda_{0}} \neq 0$. Let $\Lambda$ be the set of all such $\lambda_{0}$ and let $M_{(n)}=\bigoplus_{\lambda \in \Lambda} M_{n+\lambda}$. Then $M_{(n)}$ satisfies the condition in Definition 2.2. Thus we have the following inclusions.

$$
\begin{aligned}
\{g \text {-twisted } V \text {-modules }\} & \subset\left\{\frac{1}{T} \mathbb{N} \text {-graded weak } g \text {-twisted } V \text {-modules }\right\} \\
& \subset\{\text { weak } g \text {-twisted } V \text {-modules }\}
\end{aligned}
$$

Definition 2.4. A vertex operator algebra $V$ is said to be $g$-rational if every $\frac{1}{T} \mathbb{N}$ graded weak $g$-twisted $V$-module is semisimple, that is, a direct sum of simple $\frac{1}{T} \mathbb{N}$-graded weak $g$-twisted $V$-modules.

Let $M$ be a weak $g$-twisted $V$-module. The next lemma is a twisted version of [Li 2001, Lemma 3.12]. In fact, using the associativity formula (2-4) we can prove it by essentially the same argument as in [Li 2001].

Lemma 2.5. Let $u \in V^{r}, v \in V^{s}, w \in M$, and $k$ be a nonnegative integer such that $z^{k+r / T} Y_{M}(u, z) w \in M \llbracket z \rrbracket$. Let $p \in \frac{r}{T}+\mathbb{Z}, q \in \frac{s}{T}+\mathbb{Z}$, and $N$ be a nonnegative integer such that $z^{N+1+q} Y_{M}(v, z) w \in M \llbracket z \rrbracket$. Then

$$
u_{p} v_{q} w=\sum_{i=0}^{N} \sum_{j=0}^{\infty}\left(\begin{array}{c}
p-k-r / T \\
i
\end{array}\right)\left(\begin{array}{c}
k+r / T \\
j
\end{array}\right)\left(u_{p-k-r / T-i+j} v\right)_{q+k+r / T+i-j} w .
$$

Conversely, $\left(u_{p} v\right)_{q} w$ can be written as a linear combination of some vectors of the form $u_{i} v_{j} w$. 
Lemma 2.6. Let $u \in V^{r}, v \in V^{s}, w \in M$. Then for $p \in \mathbb{Z}$ and $q \in \frac{r+s}{T}+\mathbb{Z}$, the vector $\left(u_{p} v\right)_{q} w$ is a linear combination of $u_{i} v_{j} w$ with $i \in \frac{r}{T}+\mathbb{Z}$ and $j \in \frac{s}{T}+\mathbb{Z}$.

Proof. Let $X=\operatorname{span}\left\{u_{i} v_{j} w \mid i \in \frac{r}{T}+\mathbb{Z}, j \in \frac{s}{T}+\mathbb{Z}\right\}$. We use (2-2). Take $m \in \frac{r}{T}+\mathbb{Z}$ such that $u_{m+i} w=0$ for $i \geq 0$. Let $N \in \mathbb{Z}$ be such that $u_{N+i} v=0$ for $i>0$. If $p>N$, then $u_{p} v=0$ and the assertion is trivial. Assume that $p \leq N$. For $j=0,1, \ldots, N-p$, let $l=p+j$ and $n=q-m-j$ in (2-2). Then

$$
\sum_{i=0}^{\infty}\left(\begin{array}{c}
m \\
i
\end{array}\right)\left(u_{p+j+i} v\right)_{q-j-i} w=\sum_{i=0}^{\infty}(-1)^{i}\left(\begin{array}{c}
p+j \\
i
\end{array}\right) u_{p+m+j-i} v_{q-m-j+i} w
$$

The right hand side of this equation is contained in $X$. Consider the left hand side for each of $j=N-p, N-p-1, \ldots, 1,0$. Then we see that $\left(u_{N} v\right)_{q-N+p} w \in X$, $\left(u_{N-1} v\right)_{q-N+p+1} w \in X, \ldots$, and $\left(u_{p} v\right)_{q} w \in X$.

For subsets $A, B$ of $V$ and a subset $X$ of $M$, set $A \cdot X=\operatorname{span}\left\{u_{n} w \mid u \in A, w \in\right.$ $\left.X, n \in \frac{1}{T} \mathbb{Z}\right\}$ and $A \cdot B=\operatorname{span}\left\{u_{n} v \mid u \in A, v \in B, n \in \mathbb{Z}\right\}$. Then it follows from (2-6) that $A \cdot(B \cdot X) \subset(A \cdot B) \cdot X$ (see also [Yamauchi 2004, (2.2)]). For a vector $w \in M$, this in particular implies that $V \cdot w$ is a weak $g$-twisted $V$-submodule of $M$. If $w$ is an eigenvector for $L(0)$, then $V \cdot w$ is a direct sum of eigenspaces for $L(0)$. Each eigenspace is not necessarily of finite dimension. Thus $V \cdot w$ is not a $g$-twisted module in general. This subject was discussed in [Abe et al. 2004; Buhl 2002; Yamauchi 2004]. We will review it later in this section.

Zhu [1996] introduced an associative algebra $A(V)$ called the Zhu algebra for a vertex operator algebra $V$, which plays a crucial role in the study of representations for $V$. Later, Dong, Li and Mason [Dong et al. 1998a] constructed an associative algebra $A_{g}(V)$ called the $g$-twisted Zhu algebra in order to generalize Zhu's theory to $g$-twisted representations for $V$. The definition of $A_{g}(V)$ is similar to that of $A(V)$. Let $V, g, T$, and $V^{r}$ be as before. Roughly speaking, $A_{g}(V)=V / O_{g}(V)$ is a quotient space of $V$ with a binary operation $*_{g}$. It is in fact an associative algebra with respect to $*_{g}$. If $r \neq 0$, then $V^{r} \subset O_{g}(V)$. Thus $A_{g}(V)=\left(V^{0}+\right.$ $\left.O_{g}(V)\right) / O_{g}(V)$. For the case $g=1$, see (5-1) in Section 5.

A certain Lie algebra $V[g]$ was considered in [Dong et al. 1998a]. Any weak $g$-twisted $V$-module is a module for the Lie algebra $V[g]$ (see Lemma 5.1 of that reference). Moreover, for a $V[g]$-module $M$, the space $\Omega(M)$ of lowest-weight vectors with respect to $V[g]$ was defined. If $M$ is a weak $g$-twisted $V$-modules, then $\Omega(M)$ is the set of $w \in M$ such that $v_{\mathrm{wt}(v)-1+n} w=0$ for all homogeneous vectors $v \in V$ and $0<n \in \frac{1}{T} \mathbb{Z}$. The map $v \mapsto o(v)$ for homogeneous vectors $v \in V^{0}$ induces a representation of the associative algebra $A_{g}(V)$ on $\Omega(M)$, where $o(v)=v_{\text {wt }(v)-1}$. If $M$ is a $\frac{1}{T} \mathbb{N}$-graded weak $g$-twisted $V$-module, then the top level 
$M_{(0)}$ is contained in $\Omega(M)$. In the case where $M$ is a simple $\frac{1}{T} \mathbb{N}$-graded weak $g$ twisted $V$-module, $M_{(0)}=\Omega(M)$ and $M_{(0)}$ is a simple $A_{g}(V)$-module (see [Dong et al. 1998a, Proposition 5.4]).

For any $A_{g}(V)$-module $U$, a certain $\frac{1}{T} \mathbb{N}$-graded $V[g]$-module $M(U)$ such that $M(U)_{(0)}=U$ was defined (see [Dong et al. 1998a, (6.1)]). Let $W$ be the subspace of $M(U)$ spanned by the coefficients of

$$
\begin{aligned}
\left(z_{0}+z_{2}\right)^{\operatorname{wt}(u)-1+\delta_{r}+r / T} Y_{M}\left(u, z_{0}+z_{2}\right) Y_{M}\left(v, z_{2}\right) w \\
\\
-\left(z_{2}+z_{0}\right)^{\operatorname{wt}(u)-1+\delta_{r}+r / T} Y_{M}\left(Y\left(u, z_{0}\right) v, z_{2}\right) w
\end{aligned}
$$

for all homogeneous $u \in V^{r}, v \in V, w \in U$ (see [Dong et al. 1998a, (6.3)]). Set $\bar{M}(U)=M(U) / U(V[g]) W$, which is a quotient module of $M(U)$ by the $V[g]-$ submodule generated by $W$.

The following results will be necessary in Sections 3 and 5.

Theorem 2.7 [Dong et al. 1998a, Theorem 6.2]. $\bar{M}(U)$ is a $\frac{1}{T} \mathbb{N}$-graded weak $g$ twisted $V$-module such that its top level $\bar{M}(U)_{(0)}$ is equal to $U$ and such that it has the following universal property: for any weak $g$-twisted $V$-module $M$ and any homomorphism $\varphi: U \rightarrow \Omega(M)$ of $A_{g}(V)$-modules, there is a unique homomorphism $\bar{\varphi}: \bar{M}(U) \rightarrow M$ of weak $g$-twisted $V$-modules which is an extension of $\varphi$.

Let $J$ be the sum of all $\frac{1}{T} \mathbb{N}$-graded $V[g]$-submodules of $M(U)$ which intersect trivially with $U$. Since $M(U)_{(0)}=U$, it is a unique $\frac{1}{T} \mathbb{N}$-graded $V[g]$-submodule of $M(U)$ being maximal subject to $J \cap U=0$. The principal point is that $U(V[g]) W \subset$ $J$. Set $L(U)=M(U) / J$.

Theorem 2.8 [Dong et al. 1998a, Theorem 6.3]. $L(U)$ is a $\frac{1}{T} \mathbb{N}$-graded weak $g$ twisted $V$-module such that $\Omega(L(U)) \cong U$ as $A_{g}(V)$-modules.

Remark 2.9. If $M$ is a $\frac{1}{T} \mathbb{N}$-graded weak $g$-twisted $V$-module and $\varphi: U \rightarrow M_{(0)}$ is a homomorphism of $A_{g}(V)$-modules, then the homomorphism $\bar{\varphi}: \bar{M}(U) \rightarrow M$ of weak $g$-twisted $V$-modules in Theorem 2.7 preserves the $\frac{1}{T} \mathbb{N}$-grading. Indeed, $\bar{M}(U)=\operatorname{span}\left\{v_{n} U \mid v \in V, n \in \frac{1}{T} \mathbb{Z}\right\}$ by (2-6), since $\bar{M}(U)$ is generated by $U$ as a $\frac{1}{T} \mathbb{N}$-graded weak $g$-twisted $V$-module. By (2-5), $v_{\text {wt }(v)-1-n} \bar{M}(U)_{(0)} \subset$ $\bar{M}(U)_{(n)}$ for any homogeneous $v \in V$ and $n \in \frac{1}{T} \mathbb{Z}$. Since $\bar{M}(U)_{(0)}=U$, it follows that $\bar{M}(U)_{(n)}$ is spanned by $v_{\mathrm{wt}(v)-1-n} U$ for all homogeneous $v \in V$. Now, $\bar{\varphi}\left(v_{\mathrm{wt}(v)-1-n} U\right)=v_{\mathrm{wt}(v)-1-n} \bar{\varphi}(U)$ is contained in $v_{\mathrm{wt}(v)-1-n} M_{(0)} \subset M_{(n)}$. Hence $\bar{\varphi}\left(\bar{M}(U)_{(n)}\right) \subset M_{(n)}$ as required. In the case where both of $\bar{M}(U)$ and $M$ are ordinary $g$-twisted $V$-modules, $\bar{\varphi}$ becomes a homomorphism of ordinary $g$-twisted $V$-modules since $\bar{\varphi}$ commutes with $L(0)$. 
Lemma 2.10. Let $U$ be an $A_{g}(V)$-module. Let $S$ be a $\frac{1}{T} \mathbb{N}$-graded weak g-twisted $V$-module such that it is generated by its top level $S_{(0)}$ and such that $S_{(0)}$ is isomorphic to $U$ as an $A_{g}(V)$-module. Then there is a surjective homomorphism $S \rightarrow L(U)$ of weak g-twisted $V$-modules which preserves the $\frac{1}{T} \mathbb{N}$-grading.

Proof. By Theorem 2.7 and Remark 2.9, an isomorphism $\varphi: U \rightarrow S_{(0)}$ of $A_{g}(U)$ modules can be extended to a surjective homomorphism $\bar{\varphi}: \bar{M}(U) \rightarrow S$ of weak $g$-twisted $V$-modules which preserves the $\frac{1}{T} \mathbb{N}$-grading. The kernel $\operatorname{Ker} \bar{\varphi}$ of $\bar{\varphi}$ intersects trivially with $\bar{M}(U)_{(0)}$ and so is contained in $\bigoplus_{0<n \in \frac{1}{T} \mathbb{N}} \bar{M}(U)_{(n)}$. Let $I$ be a $\frac{1}{T} \mathbb{N}$-graded $V[g]$-submodule of $M(U)$ such that $\operatorname{Ker} \bar{\varphi}=I / U(V[g]) W$. Then $I \cap U=0$. This implies that $I \subset J$. Hence $L(U)=M(U) / J$ is a homomorphic image of $M(U) / I \cong S$.

Theorem 2.11 [Dong et al. 1998a, Theorem 7.2]. L is a functor from the category of simple $A_{g}(V)$-modules to the category of simple $\frac{1}{T} \mathbb{N}$-graded weak g-twisted $V$-modules such that $\Omega \circ L=\mathrm{id}$ and $L \circ \Omega=\mathrm{id}$.

Theorem 2.12 [Dong et al. 1998a, Theorem 8.1]. Let $V$ be a g-rational vertex operator algebra.

(1) $A_{g}(V)$ is a finite dimensional semisimple associative algebra.

(2) $V$ has only finitely many isomorphism classes of simple $\frac{1}{T} \mathbb{N}$-graded weak $g$ twisted $V$-modules.

(3) Every simple $\frac{1}{T} \mathbb{N}$-graded weak g-twisted $V$-module is an ordinary g-twisted $V$-modules.

In case of $g=1$, the above argument reduces to the untwisted case. In particular, $A_{g}(V)$ is identical with the original Zhu algebra $A(V)$ if $g=1$.

There is an important intrinsic property of a vertex operator algebra, namely, the $C_{2}$-cofiniteness. Let $C_{2}(V)=\operatorname{span}\left\{u_{-2} v \mid u, v \in V\right\}$. More generally, we set $C_{2}(M)=\operatorname{span}\left\{u_{-2} w \mid u \in V, w \in M\right\}$ for a weak $V$-module $M$. If the dimension of the quotient space $V / C_{2}(V)$ is finite, $V$ is said to be $C_{2}$-cofinite. Similarly, a weak $V$-module $M$ is said to be $C_{2}$-cofinite if $M / C_{2}(M)$ is of finite dimension. The notion of $C_{2}$-cofiniteness of a vertex operator algebra was first introduced by Zhu [1996]. The subspace $C_{2}(M)$ of a weak $V$-module $M$ was studied in [Li 1999b]. We refer the reader to [Nagatomo and Tsuchiya 2005] also.

Theorem 2.13 [Dong et al. 2000, Proposition 3.6]. If $V$ is $C_{2}$-cofinite, then $A_{g}(V)$ is of finite dimension.

If $V=\bigoplus_{n=0}^{\infty} V_{n}$ and $V_{0}=\mathbb{C} \mathbf{1}$, then $V$ is said to be of CFT type. Here $V_{n}$ denotes the homogeneous subspace of weight $n$, that is, the eigenspace of $L(0)=\omega_{1}$ with eigenvalue $n$. 
Theorem 2.14 [Yamauchi 2004, Lemma 3.3]. Suppose $V$ is $C_{2}$-cofinite and of CFT type. Choose a finite dimensional L(0)-invariant and g-invariant subspace $U$ of $V$ such that $V=U+C_{2}(V)$. Let $W$ be a weak $g$-twisted $V$-module generated by a vector $w$. Then $W$ is spanned by the vectors of the form $u_{-n_{1}}^{1} u_{-n_{2}}^{2} \cdots u_{-n_{k}}^{k} w$ with $n_{1}>n_{2}>\cdots>n_{k}>-N$ and $u^{i} \in U, i=1,2, \ldots, k$, where $N \in \frac{1}{T} \mathbb{Z}$ is a constant such that $u_{m} w=0$ for all $u \in U$ and $m \geq N$.

Theorem 2.15 [Yamauchi 2004, Corollaries 3.8 and 3.9]. Suppose $V$ is $C_{2}$-cofinite and of CFT type. Then the following assertions hold.

(1) Every weak g-twisted $V$-module is a $\frac{1}{T} \mathbb{N}$-graded weak g-twisted $V$-module.

(2) Every simple weak $g$-twisted $V$-module is a simple ordinary g-twisted $V$ module.

Remark 2.16. Suppose $V$ is $C_{2}$-cofinite and of CFT type. Let $M$ be a weak $g$ twisted $V$-module and $w^{1}, \ldots, w^{k}$ be eigenvectors of $L(0)$ in $M$. Then the weak $g$-twisted $V$-submodule $W$ generated by $w^{1}, \ldots, w^{k}$ is an ordinary $g$-twisted $V$ module. Indeed, $W$ is a direct sum of eigenspaces for $L(0)$ and each homogeneous subspace is of finite dimension by Theorem 2.14.

For the untwisted case, that is, the case $g=1$, we refer the reader to [Abe et al. 2004; Buhl 2002; Dong et al. 1997; Li 1999b]. A spanning set for a vertex operator algebra was first studied in [Gaberdiel and Neitzke 2003, Proposition 8].

\section{The fixed point subalgebra $\left(V_{\sqrt{2} A_{2}}\right)^{\tau}$}

In this section we fix notation. We tend to follow the notation in [Dong et al. 2004; Kitazume et al. 2000a; Kitazume et al. 2003] unless otherwise specified. We also recall certain properties of the lattice vertex operator algebra $V_{\sqrt{2} A_{2}}$ associated with $\sqrt{2}$ times an ordinary root lattice of type $A_{2}$ and its subalgebras (see [Dong et al. 2004; Kitazume et al. 2000a; Kitazume et al. 2003; Kitazume et al. 2000b]).

Let $\alpha_{1}, \alpha_{2}$ be the simple roots of type $A_{2}$ and set $\alpha_{0}=-\left(\alpha_{1}+\alpha_{2}\right)$. Thus $\left\langle\alpha_{i}, \alpha_{i}\right\rangle=$ 2 and $\left\langle\alpha_{i}, \alpha_{j}\right\rangle=-1$ if $i \neq j$. Set $\beta_{i}=\sqrt{2} \alpha_{i}$ and let $L=\mathbb{Z} \beta_{1}+\mathbb{Z} \beta_{2}$ be the lattice spanned by $\beta_{1}$ and $\beta_{2}$. We denote the cosets of $L$ in its dual lattice $L^{\perp}=\{\alpha \in$ $\left.\mathbb{Q} \otimes_{\mathbb{Z}} L \mid\langle\alpha, L\rangle \subset \mathbb{Z}\right\}$ as follows.

$$
\begin{gathered}
L^{0}=L, \quad L^{1}=\frac{-\beta_{1}+\beta_{2}}{3}+L, \quad L^{2}=\frac{\beta_{1}-\beta_{2}}{3}+L, \\
L_{0}=L, \quad L_{a}=\frac{\beta_{2}}{2}+L, \quad L_{b}=\frac{\beta_{0}}{2}+L, \quad L_{c}=\frac{\beta_{1}}{2}+L, \\
L^{(i, j)}=L_{i}+L^{j}
\end{gathered}
$$

for $i=0, a, b, c$ and $j=0,1,2$, where $\{0, a, b, c\} \cong \mathbb{Z}_{2} \times \mathbb{Z}_{2}$ is Klein's four-group. Note that $L^{(i, j)}, i \in\{0, a, b, c\}, j \in\{0,1,2\}$ are all the cosets of $L$ in $L^{\perp}$ and $L^{\perp} / L \cong \mathbb{Z}_{2} \times \mathbb{Z}_{2} \times \mathbb{Z}_{3}$. 
We adopt the standard notation for the vertex operator algebra $\left(V_{L}, Y(\cdot, z)\right)$ associated with the lattice $L$ (see [Frenkel et al. 1988]). In particular, $\mathfrak{h}=\mathbb{C} \otimes_{\mathbb{Z}} L$ is an abelian Lie algebra, $\hat{\mathfrak{h}}=\mathfrak{h} \otimes \mathbb{C}\left[t, t^{-1}\right] \oplus \mathbb{C} c$ is the corresponding affine Lie algebra, $M(1)=\mathbb{C}[\alpha(n) ; \alpha \in \mathfrak{h}, n<0]$, where $\alpha(n)=\alpha \otimes t^{n}$, is the unique simple $\hat{\mathfrak{h}}$-module such that $\alpha(n) 1=0$ for all $\alpha \in \mathfrak{h}$ and $n>0$ and $c=1$. As a vector space $V_{L}=M(1) \otimes \mathbb{C}[L]$ and for each $v \in V_{L}$, a vertex operator $Y(v, z)=$ $\sum_{n \in \mathbb{Z}} v_{n} z^{-n-1} \in \operatorname{End}\left(V_{L}\right) \llbracket z, z^{-1} \rrbracket$ is defined. The vector $\mathbf{1}=1 \otimes 1$ is called the vacuum vector. In our case $\langle\alpha, \beta\rangle \in 2 \mathbb{Z}$ for any $\alpha, \beta \in L$. Thus the twisted group algebra $\mathbb{C}\{L\}$ of [Frenkel et al. 1988] is naturally isomorphic to the ordinary group algebra $\mathbb{C}[L]$.

There are exactly 12 inequivalent simple $V_{L}$-modules, which are represented by $V_{L^{(i, j)}}, i=0, a, b, c$ and $j=0,1,2$ (see [Dong 1993]). We use the symbol $e^{\alpha}, \alpha \in L^{\perp}$ to denote a basis of $\mathbb{C}\left\{L^{\perp}\right\}$.

We consider the following three isometries of $(L,\langle\cdot, \cdot\rangle)$.

$$
\begin{aligned}
& \tau: \beta_{1} \rightarrow \beta_{2} \rightarrow \beta_{0} \rightarrow \beta_{1}, \\
& \sigma: \beta_{1} \rightarrow \beta_{2}, \quad \beta_{2} \rightarrow \beta_{1}, \\
& \theta: \beta_{i} \rightarrow-\beta_{i}, \quad i=1,2 .
\end{aligned}
$$

Note that $\tau$ is fixed-point-free and of order 3. The isometries $\tau, \sigma$, and $\theta$ of $L$ can be extended linearly to isometries of $L^{\perp}$. Moreover, the isometry $\tau$ lifts naturally to an automorphism of $V_{L}$ :

$$
\alpha^{1}\left(-n_{1}\right) \cdots \alpha^{k}\left(-n_{k}\right) e^{\beta} \longmapsto\left(\tau \alpha^{1}\right)\left(-n_{1}\right) \cdots\left(\tau \alpha^{k}\right)\left(-n_{k}\right) e^{\tau \beta} .
$$

By abuse of notation, we denote it by $\tau$ also. We can consider the action of $\tau$ on $V_{L^{(i, j)}}$ in a similar way. We apply the same argument to $\sigma$ and $\theta$. Our purpose is the classification of simple modules for the fixed point subalgebra $V_{L}^{\tau}=\{v \in$ $\left.V_{L} \mid \tau v=v\right\}$ of $V_{L}$ by the automorphism $\tau$.

For a simple $V_{L}$-module $\left(U, Y_{U}\right)$, let $\left(U \circ \tau, Y_{U \circ \tau}\right)$ be a new $V_{L}$-module such that $U \circ \tau=U$ as vector spaces and $Y_{U \circ \tau}(v, z)=Y_{U}(\tau v, z)$ for $v \in V_{L}$ [Dong et al. 2000]. Then $U \mapsto U \circ \tau$ induces a permutation on the set of simple $V_{L}$-modules. If $U$ and $U \circ \tau$ are equivalent $V_{L}$-modules, $U$ is said to be $\tau$-stable. The following lemma is a straightforward consequence of the definition of $V_{L^{(i, j)}}$.

Lemma 3.1. (1) $V_{L^{(0, j)}}, j=0,1,2$ are $\tau$-stable.

(2) $V_{L^{(a, j)}} \circ \tau=V_{L^{(c, j)}}, V_{L^{(c, j)}} \circ \tau=V_{L^{(b, j)}}$, and $V_{L^{(b, j)}} \circ \tau=V_{L^{(a, j)}}, j=0,1,2$.

A family of simple twisted modules for lattice vertex operator algebras was constructed in [Dong and Lepowsky 1996; Lepowsky 1985]. Following Dong and Lepowsky, three inequivalent simple $\tau$-twisted $V_{L}$-modules $\left(V_{L}^{j}(\tau), Y^{\tau}(\cdot, z)\right), j=$ 0, 1, 2 were studied in [Dong et al. 2004; Kitazume et al. 2003]. By the preceding lemma and [Dong et al. 2000, Theorem 10.2], we know that $\left(V_{L}^{j}(\tau), Y^{\tau}(\cdot, z)\right)$, 
$j=0,1,2$, are all the inequivalent simple $\tau$-twisted $V_{L}$-modules. Similarly, there are exactly three inequivalent simple $\tau^{2}$-twisted $V_{L}$-modules $\left(V_{L}^{j}\left(\tau^{2}\right), Y^{\tau^{2}}(\cdot, z)\right)$, $j=0,1,2$.

We use the same notation for $\left(V_{L}^{j}(\tau), Y^{\tau}(\cdot, z)\right)$ and $\left(V_{L}^{j}\left(\tau^{2}\right), Y^{\tau^{2}}(\cdot, z)\right)$ as in [Dong et al. 2004, Section 4]. Thus

$$
V_{L}^{j}(\tau)=S[\tau] \otimes T_{\chi_{j}}
$$

where $T_{\chi_{j}}, j=0,1,2$, is the one-dimensional representation of a certain central extension of $L$ affording the character $\chi_{j}$. Let

$$
h_{1}=\frac{1}{3}\left(\beta_{1}+\xi^{2} \beta_{2}+\xi \beta_{0}\right), \quad h_{2}=\frac{1}{3}\left(\beta_{1}+\xi \beta_{2}+\xi^{2} \beta_{0}\right) .
$$

Then $\tau h_{i}=\xi^{i} h_{i},\left\langle h_{1}, h_{1}\right\rangle=\left\langle h_{2}, h_{2}\right\rangle=0$, and $\left\langle h_{1}, h_{2}\right\rangle=2$. Moreover, $\beta_{i}=\xi^{i-1} h_{1}+$ $\xi^{2(i-1)} h_{2}, i=0,1,2$. As a vector space, $S[\tau]$ is isomorphic to a polynomial algebra with variables $h_{1}(1 / 3+n), h_{2}(2 / 3+n), n \in \mathbb{Z}_{<0}$. The isometry $\tau$ acts on $S[\tau]$ by $\tau h_{j}=\xi^{j} h_{j}$. We define the action of $\tau$ on $T_{\chi_{j}}$ to be the identity. The weight in $S[\tau]$ is given by wt $h_{i}(i / 3+n)=-i / 3-n, i=1,2$ and wt $1=1 / 9$. The weight of any element of $T_{\chi_{j}}$ is defined to be 0 . Note that the weight in $V_{L}^{j}(\tau)$ is identical with the eigenvalue for the action of the coefficient of $z^{-2}$ in the $\tau$-twisted vertex operator $Y^{\tau}(\omega, z)$, where $\omega$ denotes the Virasoro element of $V_{L}$.

The simple $\tau^{2}$-twisted $V_{L}$-modules $\left(V_{L}^{j}\left(\tau^{2}\right), Y^{\tau^{2}}(\cdot, z)\right), j=0,1,2$ are

$$
V_{L}^{j}\left(\tau^{2}\right)=S\left[\tau^{2}\right] \otimes T_{\chi_{j}^{\prime}},
$$

where $T_{\chi_{j}^{\prime}}, j=0,1,2$, are the one-dimensional representations of a certain central extension of $L$ affording the character $\chi_{j}^{\prime}$. Moreover, $S\left[\tau^{2}\right]$ is isomorphic to a polynomial algebra with variables $h_{1}^{\prime}(1 / 3+n), h_{2}^{\prime}(2 / 3+n), n \in \mathbb{Z}_{<0}$ as a vector space, where $h_{1}^{\prime}=h_{2}$ and $h_{2}^{\prime}=h_{1}$. Thus $\tau^{2} h_{i}^{\prime}=\xi^{i} h_{i}^{\prime}, i=1,2$. The action of $\tau$ on $S\left[\tau^{2}\right]$ is given by $\tau h_{i}^{\prime}=\xi^{2 i} h_{i}^{\prime}, i=1,2$. The action of $\tau$ on $T_{\chi_{j}^{\prime}}$ is defined to be the identity. The weight in $S\left[\tau^{2}\right]$ is given by wt $h_{i}^{\prime}(i / 3+n)=-i / 3-n, i=1,2$ and wt $1=1 / 9$. The weight of any element of $T_{\chi_{j}^{\prime}}$ is defined to be 0 . The weight in $V_{L}^{j}\left(\tau^{2}\right)$ is identical with the eigenvalue for the action of the coefficient of $z^{-2}$ in the $\tau^{2}$-twisted vertex operator $Y^{\tau^{2}}(\omega, z)$.

By Lemma 3.1, [Dong and Mason 1997, Theorem 4.4], and [Dong and Yamskulna 2002, Theorem 6.14],

$$
V_{L^{(0, j)}}(\varepsilon)=\left\{v \in V_{L^{(0, j)}} \mid \tau v=\xi^{\varepsilon} v\right\}, \quad j, \varepsilon=0,1,2
$$

are inequivalent simple $V_{L}^{\tau}$-modules. For each of $j=0,1,2$, we have that $V_{L^{(i, j)}}$, $i=a, b, c$ are equivalent simple $V_{L}^{\tau}$-modules. Moreover, $V_{L^{(c, j)}}, j=0,1,2$ are inequivalent simple $V_{L}^{\tau}$-modules. From [Miyamoto and Tanabe 2004, Theorem 2], 
it follows that

$$
V_{L}^{j}(\tau)(\varepsilon)=\left\{v \in V_{L}^{j}(\tau) \mid \tau v=\xi^{\varepsilon} v\right\}, \quad j, \varepsilon=0,1,2
$$

are inequivalent simple $V_{L}^{\tau}$-modules. Similar assertions hold for simple $\tau^{2}$-twisted modules, namely,

$$
V_{L}^{j}\left(\tau^{2}\right)(\varepsilon)=\left\{v \in V_{L}^{j}\left(\tau^{2}\right) \mid \tau^{2} v=\xi^{\varepsilon} v\right\}, \quad j, \varepsilon=0,1,2
$$

are inequivalent simple $V_{L}^{\tau}$-modules. In this way we obtain 30 simple $V_{L}^{\tau}$-modules. These 30 simple $V_{L}^{\tau}$-modules are inequivalent by [Miyamoto and Tanabe 2004, Theorem 2]. We summarize the result as follows.

Lemma 3.2. The following 30 simple $V_{L}^{\tau}$-modules are inequivalent.

(1) $V_{L^{(0, j)}}(\varepsilon), j, \varepsilon=0,1,2$,

(2) $V_{L^{(c, j)}}, j=0,1,2$,

(3) $V_{L}^{j}(\tau)(\varepsilon), j, \varepsilon=0,1,2$,

(4) $V_{L}^{j}\left(\tau^{2}\right)(\varepsilon), j, \varepsilon=0,1,2$.

We consider the structure of $V_{L}^{\tau}$ in detail. Set

$$
x(\alpha)=e^{\sqrt{2} \alpha}+e^{-\sqrt{2} \alpha}, \quad y(\alpha)=e^{\sqrt{2} \alpha}-e^{-\sqrt{2} \alpha}, \quad w(\alpha)=\frac{1}{2} \alpha(-1)^{2}-x(\alpha)
$$

for $\alpha \in\left\{ \pm \alpha_{0}, \pm \alpha_{1}, \pm \alpha_{2}\right\}$ and let

$$
\begin{gathered}
\omega=\frac{1}{6}\left(\alpha_{1}(-1)^{2}+\alpha_{2}(-1)^{2}+\alpha_{0}(-1)^{2}\right), \\
\tilde{\omega}^{1}=\frac{1}{5}\left(w\left(\alpha_{1}\right)+w\left(\alpha_{2}\right)+w\left(\alpha_{0}\right)\right), \quad \tilde{\omega}^{2}=\omega-\tilde{\omega}^{1}, \\
\omega^{1}=\frac{1}{4} w\left(\alpha_{1}\right), \quad \omega^{2}=\tilde{\omega}^{1}-\omega^{1} .
\end{gathered}
$$

Then $\omega$ is the Virasoro element of $V_{L}$ and $\tilde{\omega}^{1}, \tilde{\omega}^{2}$ are mutually orthogonal conformal vectors of central charge $6 / 5,4 / 5$ respectively. The subalgebra $\operatorname{Vir}\left(\tilde{\omega}^{i}\right)$ generated by $\tilde{\omega}^{i}$ is isomorphic to the Virasoro vertex operator algebra of given central charge, namely, $\operatorname{Vir}\left(\tilde{\omega}^{1}\right) \cong L(6 / 5,0)$ and $\operatorname{Vir}\left(\tilde{\omega}^{2}\right) \cong L(4 / 5,0)$. Moreover, $\tilde{\omega}^{1}$ is a sum of two conformal vectors $\omega^{1}$ and $\omega^{2}$ of central charge $1 / 2$ and 7/10 respectively and $\omega^{1}, \omega^{2}$ and $\tilde{\omega}^{2}$ are mutually orthogonal. Note that $\tilde{\omega}^{2}$ was denoted by $\omega^{3}$ in [Dong et al. 2004; Kitazume et al. 2000a; Kitazume et al. 2003; Kitazume et al. 2000b]. Such a decomposition of the Virasoro element of a lattice vertex operator algebra into a sum of mutually orthogonal conformal vectors was first studied in [Dong et al. 1998b].

Set

$$
M_{k}^{i}=\left\{v \in V_{L_{i}} \mid\left(\tilde{\omega}^{2}\right)_{1} v=0\right\}, \quad W_{k}^{i}=\left\{v \in V_{L_{i}} \mid\left(\tilde{\omega}^{2}\right)_{1} v=\frac{2}{5} v\right\}, \quad i=0, a, b, c,
$$




$$
\begin{aligned}
& M_{t}^{j}=\left\{v \in V_{L^{j}} \mid\left(\omega^{1}\right)_{1} v=\left(\omega^{2}\right)_{1} v=0\right\}, \\
& W_{t}^{j}=\left\{v \in V_{L^{j}} \mid\left(\omega^{1}\right)_{1} v=0,\left(\omega^{2}\right)_{1} v=\frac{3}{5} v\right\}, \quad j=0,1,2 .
\end{aligned}
$$

Then $M_{k}^{0}$ and $M_{t}^{0}$ are simple vertex operator algebras. Moreover, $\left\{M_{k}^{i}, W_{k}^{i} ; i=\right.$ $0, a, b, c\}$ and $\left\{M_{t}^{j}, W_{t}^{j} ; j=0,1,2\right\}$ are complete sets of representatives of isomorphism classes of simple modules for $M_{k}^{0}$ and $M_{t}^{0}$, respectively (see [Kitazume et al. 2000a; Kitazume et al. 2000b; Lam and Yamada 2000]). As $\operatorname{Vir}\left(\omega^{1}\right) \otimes$ $\operatorname{Vir}\left(\omega^{2}\right)$-modules,

$$
\begin{aligned}
& \left.M_{k}^{0} \cong\left(L\left(\frac{1}{2}, 0\right) \otimes L\left(\frac{7}{10}, 0\right)\right) \oplus\left(L\left(\frac{1}{2}, \frac{1}{2}\right) \otimes L \frac{7}{10}, \frac{3}{2}\right)\right), \\
& M_{k}^{a} \cong M_{k}^{b} \cong L\left(\frac{1}{2}, \frac{1}{16}\right) \otimes L\left(\frac{7}{10}, \frac{7}{16}\right), \\
& M_{k}^{c} \cong\left(L\left(\frac{1}{2}, \frac{1}{2}\right) \otimes L\left(\frac{7}{10}, 0\right)\right) \oplus\left(L\left(\frac{1}{2}, 0\right) \otimes L\left(\frac{7}{10}, \frac{3}{2}\right)\right), \\
& W_{k}^{0} \cong\left(L\left(\frac{1}{2}, 0\right) \otimes L\left(\frac{7}{10}, \frac{3}{5}\right)\right) \oplus\left(L\left(\frac{1}{2}, \frac{1}{2}\right) \otimes L\left(\frac{7}{10}, \frac{1}{10}\right)\right), \\
& W_{k}^{a} \cong W_{k}^{b} \cong L\left(\frac{1}{2}, \frac{1}{16}\right) \otimes L\left(\frac{7}{10}, \frac{3}{80}\right), \\
& W_{k}^{c} \cong\left(L\left(\frac{1}{2}, \frac{1}{2}\right) \otimes L\left(\frac{7}{10}, \frac{3}{5}\right)\right) \oplus\left(L\left(\frac{1}{2}, 0\right) \otimes L\left(\frac{7}{10}, \frac{1}{10}\right)\right),
\end{aligned}
$$

and as $\operatorname{Vir}\left(\tilde{\omega}^{2}\right)$-modules,

$$
\begin{array}{ll}
M_{t}^{0} \cong L\left(\frac{4}{5}, 0\right) \oplus L\left(\frac{4}{5}, 3\right), & M_{t}^{1} \cong M_{t}^{2} \cong L\left(\frac{4}{5}, \frac{2}{3}\right), \\
W_{t}^{0} \cong L\left(\frac{4}{5}, \frac{2}{5}\right) \oplus L\left(\frac{4}{5}, \frac{7}{5}\right), & W_{t}^{1} \cong W_{t}^{2} \cong L\left(\frac{4}{5}, \frac{1}{15}\right) .
\end{array}
$$

Furthermore,

$$
V_{L^{(i, j)}} \cong\left(M_{k}^{i} \otimes M_{t}^{j}\right) \oplus\left(W_{k}^{i} \otimes W_{t}^{j}\right)
$$

as $M_{k}^{0} \otimes M_{t}^{0}$-modules. In particular,

$$
V_{L} \cong\left(M_{k}^{0} \otimes M_{t}^{0}\right) \oplus\left(W_{k}^{0} \otimes W_{t}^{0}\right) .
$$

Note that $M_{t}^{j}=\left\{v \in V_{L^{j}} \mid\left(\tilde{\omega}^{1}\right)_{1} v=0\right\}$ and that $M_{k}^{0}, W_{k}^{0}$ and $M_{t}^{j}, j=0,1,2$ are $\tau$-invariant. However, $W_{t}^{j}, j=0,1,2$ are not $\tau$-invariant.

The fusion rules for $M_{k}^{0}$ and $M_{t}^{0}$ were determined in [Lam and Yamada 2000] and [Miyamoto 2001], respectively. They are

$$
M_{k}^{i} \times M_{k}^{j}=M_{k}^{i+j}, \quad M_{k}^{i} \times W_{k}^{j}=W_{k}^{i+j}, \quad W_{k}^{i} \times W_{k}^{j}=M_{k}^{i+j}+W_{k}^{i+j}
$$

for $i, j=0, a, b, c$ and

$$
M_{t}^{i} \times M_{t}^{j}=M_{t}^{i+j}, \quad M_{t}^{i} \times W_{t}^{j}=W_{t}^{i+j}, \quad W_{t}^{i} \times W_{t}^{j}=M_{t}^{i+j}+W_{t}^{i+j}
$$

for $i, j=0,1,2$. 
The following two weight-three vectors are important.

$$
\begin{aligned}
& J=w\left(\alpha_{1}\right)_{0} w\left(\alpha_{2}\right)-w\left(\alpha_{2}\right)_{0} w\left(\alpha_{1}\right) \\
& =-\frac{1}{6}\left(\beta_{1}(-2)\left(\beta_{2}-\beta_{0}\right)(-1)+\beta_{2}(-2)\left(\beta_{0}-\beta_{1}\right)(-1)+\beta_{0}(-2)\left(\beta_{1}-\beta_{2}\right)(-1)\right) \\
& -\left(\beta_{2}-\beta_{0}\right)(-1) y\left(\alpha_{1}\right)-\left(\beta_{0}-\beta_{1}\right)(-1) y\left(\alpha_{2}\right)-\left(\beta_{1}-\beta_{2}\right)(-1) y\left(\alpha_{0}\right), \\
& K=-\frac{1}{9}\left(\beta_{1}-\beta_{2}\right)(-1)\left(\beta_{2}-\beta_{0}\right)(-1)\left(\beta_{0}-\beta_{1}\right)(-1) \\
& +\left(\beta_{2}-\beta_{0}\right)(-1) x\left(\alpha_{1}\right)+\left(\beta_{0}-\beta_{1}\right)(-1) x\left(\alpha_{2}\right)+\left(\beta_{1}-\beta_{2}\right)(-1) x\left(\alpha_{0}\right) .
\end{aligned}
$$

Let $M(0)=\left(M_{k}^{0}\right)^{\tau}=\left\{u \in M_{k}^{0} \mid \tau u=u\right\}$. The vertex operator algebra $M(0)$ was studied in [Dong et al. 2004]. Among other things, the classification of simple modules, the rationality and the $C_{2}$-cofiniteness for $M(0)$ were established. It is known that $M(0)$ is a $W_{3}$ algebra of central charge $6 / 5$ with the Virasoro element $\tilde{\omega}^{1}$. In fact, $M(0)$ is generated by $\tilde{\omega}^{1}$ and $J$. The following equations hold [Dong et al. 2004, (3.1)].

$$
\begin{aligned}
& J_{5} J=-84 \cdot \mathbf{1}, \\
& J_{4} J=0, \\
& J_{3} J=-420 \tilde{\omega}^{1}, \\
& J_{2} J=-210\left(\tilde{\omega}^{1}\right)_{0} \tilde{\omega}^{1}, \\
& J_{1} J=9\left(\tilde{\omega}^{1}\right)_{0}\left(\tilde{\omega}^{1}\right)_{0} \tilde{\omega}^{1}-240\left(\tilde{\omega}^{1}\right)_{-1} \tilde{\omega}^{1}, \\
& J_{0} J=22\left(\tilde{\omega}^{1}\right)_{0}\left(\tilde{\omega}^{1}\right)_{0}\left(\tilde{\omega}^{1}\right)_{0} \tilde{\omega}^{1}-120\left(\tilde{\omega}^{1}\right)_{0}\left(\tilde{\omega}^{1}\right)_{-1} \tilde{\omega}^{1} .
\end{aligned}
$$

Let $L^{1}(n)=\left(\tilde{\omega}^{1}\right)_{n+1}$ and $J(n)=J_{n+2}$ for $n \in \mathbb{Z}$, so that the weight of these operators is wt $L^{1}(n)=$ wt $J(n)=-n$. Then

$$
\begin{gathered}
{\left[L^{1}(m), L^{1}(n)\right]=(m-n) L^{1}(m+n)+\frac{m^{3}-m}{12} \cdot \frac{6}{5} \cdot \delta_{m+n, 0},} \\
{\left[L^{1}(m), J(n)\right]=(2 m-n) J(m+n),}
\end{gathered}
$$

(3-11) $\quad[J(m), J(n)]$

$$
\begin{array}{r}
=(m-n)(22(m+n+2)(m+n+3)+35(m+2)(n+2)) L^{1}(m+n) \\
-120(m-n)\left(\sum_{k \leq-2} L^{1}(k) L^{1}(m+n-k)+\sum_{k \geq-1} L^{1}(m+n-k) L^{1}(k)\right) \\
-\frac{7}{10} m\left(m^{2}-1\right)\left(m^{2}-4\right) \delta_{m+n, 0} .
\end{array}
$$

The vertex operator algebra $M_{t}^{0}$ is known as a 3-State Potts model. It is a $W_{3}$ algebra of central charge $4 / 5$ with the Virasoro element $\tilde{\omega}^{2}$ and is generated by 
$\tilde{\omega}^{2}$ and $K$. Both of $\tilde{\omega}^{2}$ and $K$ are fixed by $\tau$, so that $\tau$ is the identity on $M_{t}^{0}$. The rationality of $M_{t}^{0}$ was established in [Kitazume et al. 2000b] and the $C_{2}$-cofiniteness of $M_{t}^{0}$ follows from [Buhl 2002]. By a direct calculation, we can verify that

$$
\begin{aligned}
& K_{5} K=104 \cdot \mathbf{1}, \\
& K_{4} K=0, \\
& K_{3} K=780 \tilde{\omega}^{2}, \\
& K_{2} K=390\left(\tilde{\omega}^{2}\right)_{0} \tilde{\omega}^{2}, \\
& K_{1} K=-27\left(\tilde{\omega}^{2}\right)_{0}\left(\tilde{\omega}^{2}\right)_{0} \tilde{\omega}^{2}+480\left(\tilde{\omega}^{2}\right)_{-1} \tilde{\omega}^{2}, \\
& K_{0} K=-46\left(\tilde{\omega}^{2}\right)_{0}\left(\tilde{\omega}^{2}\right)_{0}\left(\tilde{\omega}^{2}\right)_{0} \tilde{\omega}^{2}+240\left(\tilde{\omega}^{2}\right)_{0}\left(\tilde{\omega}^{2}\right)_{-1} \tilde{\omega}^{2} .
\end{aligned}
$$

Let $L^{2}(n)=\left(\tilde{\omega}^{2}\right)_{n+1}$ and $K(n)=K_{n+2}$ for $n \in \mathbb{Z}$. Then

$$
\begin{gathered}
{\left[L^{2}(m), L^{2}(n)\right]=(m-n) L^{2}(m+n)+\frac{m^{3}-m}{12} \cdot \frac{4}{5} \cdot \delta_{m+n, 0},} \\
{\left[L^{2}(m), K(n)\right]=(2 m-n) K(m+n),} \\
{[K(m), K(n)]} \\
=-(m-n)(46(m+n+2)(m+n+3)+65(m+2)(n+2)) L^{2}(m+n) \\
+240(m-n)\left(\sum_{k \leq-2} L^{2}(k) L^{2}(m+n-k)+\sum_{k \geq-1} L^{2}(m+n-k) L^{2}(k)\right) \\
\quad+\frac{13}{15} m\left(m^{2}-1\right)\left(m^{2}-4\right) \delta_{m+n, 0} .
\end{gathered}
$$

Remark 3.3. Let $L_{n}=L^{1}(n), W_{n}=\sqrt{-1 / 210} J(n)$, and $c=6 / 5$. Then the commutation relations above coincide with (2.1) and (2.2) of [Bouwknegt et al. 1996]. The same commutation relations also hold if we set $L_{n}=L^{2}(n), W_{n}=$ $K(n) / \sqrt{390}$, and $c=4 / 5$.

Let us review the 20 inequivalent simple $M(0)$-modules studied in [Dong et al. 2004]. Among those simple $M(0)$-modules, eight of them appear in simple $M_{k}^{0}$ modules, namely,

$$
M(\varepsilon)=\left\{u \in M_{k}^{0} \mid \tau u=\xi^{\varepsilon} u\right\}, \quad W(\varepsilon)=\left\{u \in W_{k}^{0} \mid \tau u=\xi^{\varepsilon} u\right\}
$$

for $\varepsilon=0,1,2, M_{k}^{c}$ and $W_{k}^{c}$. The remaining 12 simple $M(0)$-modules appear in simple $\tau$-twisted or $\tau^{2}$-twisted $V_{L}$-modules. Let

$$
\begin{aligned}
& M_{T}(\tau)(\varepsilon)=\left\{u \in V_{L}^{0}(\tau) \mid\left(\tilde{\omega}^{2}\right)_{1} u=0, \tau u=\xi^{\varepsilon} u\right\}, \\
& W_{T}(\tau)(\varepsilon)=\left\{u \in V_{L}^{0}(\tau) \mid\left(\tilde{\omega}^{2}\right)_{1} u=\frac{2}{5} u, \tau u=\xi^{\varepsilon} u\right\} .
\end{aligned}
$$


Then $M_{T}(\tau)(\varepsilon), W_{T}(\tau)(\varepsilon), \varepsilon=0,1,2$ are inequivalent simple $M(0)$-modules. Similarly,

$$
\begin{aligned}
& M_{T}\left(\tau^{2}\right)(\varepsilon)=\left\{u \in V_{L}^{0}\left(\tau^{2}\right) \mid\left(\tilde{\omega}^{2}\right)_{1} u=0, \tau^{2} u=\xi^{\varepsilon} u\right\}, \\
& W_{T}\left(\tau^{2}\right)(\varepsilon)=\left\{u \in V_{L}^{0}\left(\tau^{2}\right) \mid\left(\tilde{\omega}^{2}\right)_{1} u=\frac{2}{5} u, \tau^{2} u=\xi^{\varepsilon} u\right\}
\end{aligned}
$$

for $\varepsilon=0,1,2$ are inequivalent simple $M(0)$-modules. In [Dong et al. 2004], it was shown that $M(\varepsilon), W(\varepsilon), M_{k}^{c}, W_{k}^{c}, M_{T}(\tau)(\varepsilon), W_{T}(\tau)(\varepsilon), M_{T}\left(\tau^{2}\right)(\varepsilon)$, and $W_{T}\left(\tau^{2}\right)(\varepsilon), \varepsilon=0,1,2$ form a complete set of representatives of isomorphism classes of simple $M(0)$-modules.

Let us describe the structure of the fixed point subalgebra $V_{L}^{\tau}$. By the definition of $M(0)$ and $M_{t}^{0}$, we see that $V_{L}^{\tau} \supset M(0) \otimes M_{t}^{0}$. Since both of $M(0)$ and $M_{t}^{0}$ are rational, $M(0) \otimes M_{t}^{0}$ is also rational. Thus $V_{L}(\varepsilon)=\left\{u \in V_{L} \mid \tau u=\xi^{\varepsilon} u\right\}, \varepsilon=0,1,2$ can be decomposed into a direct sum of simple modules for $M(0) \otimes M_{t}^{0}$. Any simple module for $M(0) \otimes M_{t}^{0}$ is of the form $A \otimes B$, where $A$ and $B$ are simple modules for $M(0)$ and $M_{t}^{0}$, respectively. By (3-5), it follows that $B \cong M_{t}^{0}$ or $W_{t}^{0}$. Moreover, $V_{L}(\varepsilon)$ contains the simple $M(0)$-modules $M(\varepsilon)$ and $W(\varepsilon)$. The eigenvalues of $\left(\tilde{\omega}^{1}\right)_{1}$ in $M(\varepsilon)$ (resp. $W(\varepsilon)$ ) are integers (resp. of the form $3 / 5+n$, $n \in \mathbb{Z}$ ), while the eigenvalues of $\left(\tilde{\omega}^{2}\right)_{1}$ in $M_{t}^{0}$ (resp. $W_{t}^{0}$ ) are integers (resp. of the form $2 / 5+n, n \in \mathbb{Z})$. Since the eigenvalues of $\omega_{1}=\left(\tilde{\omega}^{1}\right)_{1}+\left(\tilde{\omega}^{2}\right)_{1}$ in $V_{L}$ are integers, we conclude that

$$
V_{L}(\varepsilon) \cong\left(M(\varepsilon) \otimes M_{t}^{0}\right) \oplus\left(W(\varepsilon) \otimes W_{t}^{0}\right)
$$

as $M(0) \otimes M_{t}^{0}$-modules, $\varepsilon=0,1,2$. In particular,

$$
V_{L}^{\tau} \cong\left(M(0) \otimes M_{t}^{0}\right) \oplus\left(W(0) \otimes W_{t}^{0}\right) .
$$

From now on we set $M^{0}=M(0) \otimes M_{t}^{0}$ and $W^{0}=W(0) \otimes W_{t}^{0}$. Thus $V_{L}^{\tau}=$ $V_{L}(0) \cong M^{0} \oplus W^{0}$. Let

$$
P=y\left(\alpha_{1}\right)+y\left(\alpha_{2}\right)+y\left(\alpha_{0}\right) .
$$

Then we can verify that $\left(\tilde{\omega}^{1}\right)_{n} P=\left(\tilde{\omega}^{2}\right)_{n} P=0$ for $n \geq 2$, $\left(\tilde{\omega}^{1}\right)_{1} P=(8 / 5) P$, and $\left(\tilde{\omega}^{2}\right)_{1} P=(2 / 5) P$. Moreover, $J_{n} P=K_{n} P=0$ for $n \geq 2$. Thus $W^{0}$ is a simple $M^{0}$ module with $P$ a highest-weight vector of weight $(8 / 5,2 / 5)$. The vertex operator algebra $V_{L}^{\tau}$ is generated by $\tilde{\omega}^{1}, \tilde{\omega}^{2}, J, K$ and $P$.

Theorem 3.4. $V_{L}^{\tau}$ is a simple $C_{2}$-cofinite vertex operator algebra.

Proof. We know that $M(0)$ and $M_{t}^{0}$ are $C_{2}$-cofinite. Thus $M^{0}$ is also $C_{2}$-cofinite. Since $W^{0}$ is generated by $P$ as an $M^{0}$-module, it follows from [Buhl 2002] that $V_{L}^{\tau}$ is $C_{2}$-cofinite. By [Dong and Mason 1997, Theorem 4.4], $V_{L}^{\tau}$ is simple. 
Following the outline of the argument in [Dong et al. 2004; Kitazume et al. 2003], we discuss the structure of the simple $\tau$-twisted $V_{L}$-modules $V_{L}^{j}(\tau), j=$ $0,1,2$ as $\tau$-twisted $M_{k}^{0} \otimes M_{t}^{0}$-modules. Furthermore, we correct an error in [Dong et al. 2004; Kitazume et al. 2003] concerning a decomposition of $V_{L}^{j}(\tau)$ for $j=$ 1,2 . We first consider $V_{L}^{0}(\tau)$. Let $0 \neq v \in T_{\chi_{0}}$ and 1 be the identity of $S[\tau]$. Then $1 \otimes v \in S[\tau] \otimes T_{\chi_{0}}=V_{L}^{0}(\tau)$. Since $M_{t}^{0} \subset V_{L}^{\tau}$, we can decompose $V_{L}^{0}(\tau)$ into a direct sum of simple $M_{t}^{0}$-modules. By a direct calculation, we can verify that

$$
\left(\tilde{\omega}^{2}\right)_{1}(1 \otimes v)=0, \quad\left(\tilde{\omega}^{2}\right)_{1}\left(h_{2}\left(-\frac{1}{3}\right) \otimes v\right)=\frac{2}{5} h_{2}\left(-\frac{1}{3}\right) \otimes v .
$$

Thus we see that $M_{t}^{0}$ and $W_{t}^{0}$ appear as direct summands. Since $V_{L}^{0}(\tau)$ is simple as a $\tau$-twisted $V_{L}$-module, (3-5) and the fusion rule $W_{t}^{0} \times W_{t}^{0}=M_{t}^{0}+W_{t}^{0}$ (see (3-7)) imply that any simple $M_{t}^{0}$-submodule of $V_{L}^{0}(\tau)$ is isomorphic to $M_{t}^{0}$ or $W_{t}^{0}$. Hence

$$
V_{L}^{0}(\tau) \cong\left(M_{T}^{0}(\tau) \otimes M_{t}^{0}\right) \oplus\left(W_{T}^{0}(\tau) \otimes W_{t}^{0}\right)
$$

as $\tau$-twisted $M_{k}^{0} \otimes M_{t}^{0}$-modules, where

$$
\begin{aligned}
& M_{T}^{0}(\tau)=\left\{u \in V_{L}^{0}(\tau) \mid\left(\tilde{\omega}^{2}\right)_{1} u=0\right\}, \\
& W_{T}^{0}(\tau)=\left\{u \in V_{L}^{0}(\tau) \mid\left(\tilde{\omega}^{2}\right)_{1} u=\frac{2}{5} u\right\} .
\end{aligned}
$$

The $\tau$-twisted $M_{k}^{0}$-modules $M_{T}^{0}(\tau)$ and $W_{T}^{0}(\tau)$ are simple. Indeed, if $N$ is a $\tau$ twisted $M_{k}^{0}$-submodule of $M_{T}^{0}(\tau)$, then $N \otimes M_{t}^{0}$ is a $\tau$-twisted $M_{k}^{0} \otimes M_{t}^{0}$-submodule of $M_{T}^{0}(\tau) \otimes M_{t}^{0}$. By (2-6), $V_{L} \cdot\left(N \otimes M_{t}^{0}\right)=\operatorname{span}\left\{a_{n}\left(N \otimes M_{t}^{0}\right) \mid a \in V_{L}, n \in \mathbb{Q}\right\}$ is a $\tau$-twisted $V_{L}$-submodule of $V_{L}^{0}(\tau)$. The fusion rule $W_{t}^{0} \times M_{t}^{0}=W_{t}^{0}$ and (3-5) imply that $V_{L} \cdot\left(N \otimes M_{t}^{0}\right)$ is contained in $\left(N \otimes M_{t}^{0}\right) \oplus\left(W_{T}^{0}(\tau) \otimes W_{t}^{0}\right)$. Since $V_{L}^{0}(\tau)$ is a simple $\tau$-twisted $V_{L}$-module, we conclude that $M_{T}^{0}(\tau)$ is a simple $\tau$-twisted $M_{k}^{0}$-module.

Because of the fusion rule $W_{t}^{0} \times W_{t}^{0}=M_{t}^{0}+W_{t}^{0}$, we can not apply a similar argument to $W_{T}^{0}(\tau)$. Note that there are at most two inequivalent simple $\tau$-twisted $M_{k}^{0}$-modules by [Dong et al. 2004, Lemma 4.1] and [Dong et al. 2000, Theorem 10.2]. Note also that a weight in $M_{T}^{0}(\tau)$ or in $W_{T}^{0}(\tau)$ means an eigenvalue of $\left(\tilde{\omega}^{1}\right)_{1}$. First several terms of the characters of $M_{T}^{0}(\tau)$ and $W_{T}^{0}(\tau)$ can be calculated easily from (3-18) (see [Dong et al. 2004]).

$$
\begin{aligned}
& \operatorname{ch} M_{T}^{0}(\tau)=q^{1 / 9}+q^{1 / 9+2 / 3}+q^{1 / 9+1}+q^{1 / 9+4 / 3}+\cdots, \\
& \operatorname{ch} W_{T}^{0}(\tau)=q^{2 / 45}+q^{2 / 45+1 / 3}+q^{2 / 45+2 / 3}+q^{2 / 45+1}+\cdots .
\end{aligned}
$$

Suppose $W_{T}^{0}(\tau)$ is not a simple $\tau$-twisted $M_{k}^{0}$-module. Let $N$ be the $\tau$-twisted $M_{k}^{0}$-submodule of $W_{T}^{0}(\tau)$ generated by the top level of $W_{T}^{0}(\tau)$. Then the top level of $N$ is a one dimensional space of weight 2/45. If $N$ is not a simple $\tau$-twisted $M_{k}^{0}$ module, then the sum $U$ of all proper $\tau$-twisted $M_{k}^{0}$-submodules of $N$ is a unique 
maximal $\tau$-twisted $M_{k}^{0}$-submodule of $N$. The quotient $N / U$ is a simple $\tau$-twisted $M_{k}^{0}$-module whose top level is of weight 2/45. Denote the top level of $U$ by $U_{\lambda}$, where the weight $\lambda$ is $2 / 45+n / 3$ for some $1 \leq n \in \mathbb{Z}$. Consider the $\tau$-twisted Zhu algebra $A_{\tau}\left(M_{k}^{0}\right)$ of $M_{k}^{0}$. Since $U_{\lambda}$ is a finite dimensional $A_{\tau}\left(M_{k}^{0}\right)$-module, we can choose a simple $A_{\tau}\left(M_{k}^{0}\right)$-submodule $S$ of $U_{\lambda}$. By [Dong et al. 1998a, Proposition 5.4 and Theorem 7.2], there is a simple $\frac{1}{3} \mathbb{N}$-graded weak $\tau$-twisted $M_{k}^{0}$-module $R$ with top level $R_{\lambda}$ being isomorphic to $S$ as an $A_{\tau}\left(M_{k}^{0}\right)$-module. It follows from [Yamauchi 2004, Corollary 3.8] that $R$ is in fact a simple $\tau$-twisted $M_{k}^{0}$-module. Here we note that $M_{k}^{0}$ is $C_{2}$-cofinite and of CFT type by its structure (3-2). Since the top levels of $M_{T}^{0}(\tau), N / U$, and $R$ have different weight, they are inequivalent simple $\tau$-twisted $M_{k}^{0}$-modules. If $N$ is a simple $\tau$-twisted $M_{k}^{0}$-module, then it is not equal to $W_{T}^{0}(\tau)$ by our assumption. The quotient $W_{T}^{0}(\tau) / N$ is a $\tau$-twisted $M_{k}^{0}$ module and the weight of its top level, say $\mu$ is $2 / 45+m / 3$ for some $1 \leq m \in \mathbb{Z}$. By a similar argument as above, we see that there is a simple $\tau$-twisted $M_{k}^{0}$-module whose top level is of weight $\mu$. Hence we have three inequivalent simple $\tau$-twisted $M_{k}^{0}$-modules in both cases. This contradicts the fact that there are at most two inequivalent simple $\tau$-twisted $M_{k}^{0}$-modules. Thus $W_{T}^{0}(\tau)$ is a simple $\tau$-twisted $M_{k}^{0}$-module.

Next, let $0 \neq v \in T_{\chi_{j}}, j=1$, 2. From the definition of $V_{L}^{j}(\tau)$ in [Dong et al. 2004; Kitazume et al. 2003], we can calculate that

$$
\left(\tilde{\omega}^{2}\right)_{1}(1 \otimes v)=\frac{1}{15}(1 \otimes v), \quad\left(\tilde{\omega}^{2}\right)_{1} u^{j}=\frac{2}{3} u^{j},
$$

where $u^{j}=h_{1}\left(-\frac{2}{3}\right) \otimes v-(-1)^{j} \sqrt{-3} h_{2}\left(-\frac{1}{3}\right)^{2} \otimes v$. Thus $M_{t}^{1}$ or $M_{t}^{2}$ and $W_{t}^{1}$ or $W_{t}^{2}$ appear as $M_{t}^{0}$-submodules of $V_{L}^{j}(\tau)$. In order to distinguish $M_{t}^{1}$ and $M_{t}^{2}$ (resp. $W_{t}^{1}$ and $W_{t}^{2}$ ), we need to know the action of $K_{2}$ on these vectors (see [Kitazume et al. 2000 b]). By a direct calculation, we can verify that

$$
K_{2}(1 \otimes v)=-(-1)^{j} \frac{2}{9}(1 \otimes v), \quad K_{2} u^{j}=(-1)^{j} \frac{52}{9} u^{j} .
$$

Hence $M_{t}^{3-j}$ and $W_{t}^{3-j}$ appear in $V_{L}^{j}(\tau)$ for $j=1,2$. Let

$$
\begin{aligned}
& M_{T}^{j}(\tau)=\left\{u \in V_{L}^{j}(\tau) \mid\left(\tilde{\omega}^{2}\right)_{1} u=\frac{2}{3} u\right\}, \\
& W_{T}^{j}(\tau)=\left\{u \in V_{L}^{j}(\tau) \mid\left(\tilde{\omega}^{2}\right)_{1} u=\frac{1}{15} u\right\}, \quad j=1,2 .
\end{aligned}
$$

Then, $V_{L}^{j}(\tau) \cong\left(M_{T}^{j}(\tau) \otimes M_{t}^{3-j}\right) \oplus\left(W_{T}^{j}(\tau) \otimes W_{t}^{3-j}\right)$ as $\tau$-twisted $M_{k}^{0} \otimes M_{t}^{0}$ modules for $j=1,2$. Moreover, $M_{T}^{j}(\tau)$ and $W_{T}^{j}(\tau), j=1,2$ are simple $\tau$-twisted $M_{k}^{0}$-modules.

Recall that there are at most two inequivalent simple $\tau$-twisted $M_{k}^{0}$-modules. Looking at the smallest weight of $M_{T}^{j}(\tau)$ and $W_{T}^{j}(\tau)$, we see that the $M_{T}^{j}(\tau)$, $j=0,1,2$ are equivalent, and the $W_{T}^{j}(\tau), j=0,1,2$ are equivalent, but $M_{T}^{0}(\tau)$ and 
$W_{T}^{0}(\tau)$ are not equivalent. For simplicity, set $M_{T}(\tau)=M_{T}^{0}(\tau)$ and $W_{T}(\tau)=W_{T}^{0}(\tau)$. Then

$$
\begin{aligned}
& V_{L}^{0}(\tau) \cong\left(M_{T}(\tau) \otimes M_{t}^{0}\right) \oplus\left(W_{T}(\tau) \otimes W_{t}^{0}\right), \\
& V_{L}^{j}(\tau) \cong\left(M_{T}(\tau) \otimes M_{t}^{3-j}\right) \oplus\left(W_{T}(\tau) \otimes W_{t}^{3-j}\right), \quad j=1,2,
\end{aligned}
$$

as $\tau$-twisted $M_{k}^{0} \otimes M_{t}^{0}$-modules.

The structure of the simple $\tau^{2}$-twisted $V_{L}$-module $V_{L}^{j}\left(\tau^{2}\right), j=0,1,2$ as a $\tau^{2}$ twisted $M_{k}^{0} \otimes M_{t}^{0}$-module is similar to that of the case for $V_{L}^{j}(\tau)$. Let $0 \neq v \in T_{\chi_{0}^{\prime}}$ and let 1 be the identity of $S\left[\tau^{2}\right]$. Then

$$
\left(\tilde{\omega}^{2}\right)_{1}(1 \otimes v)=0, \quad\left(\tilde{\omega}^{2}\right)_{1}\left(h_{2}^{\prime}\left(-\frac{1}{3}\right) \otimes v\right)=\frac{2}{5} h_{2}^{\prime}\left(-\frac{1}{3}\right) \otimes v
$$

and so

$$
V_{L}^{0}\left(\tau^{2}\right) \cong\left(M_{T}^{0}\left(\tau^{2}\right) \otimes M_{t}^{0}\right) \oplus\left(W_{T}^{0}\left(\tau^{2}\right) \otimes W_{t}^{0}\right)
$$

as $\tau^{2}$-twisted $M_{k}^{0} \otimes M_{t}^{0}$-modules, where

$$
\begin{aligned}
& M_{T}^{0}\left(\tau^{2}\right)=\left\{u \in V_{L}^{0}\left(\tau^{2}\right) \mid\left(\tilde{\omega}^{2}\right)_{1} u=0\right\}, \\
& W_{T}^{0}\left(\tau^{2}\right)=\left\{u \in V_{L}^{0}\left(\tau^{2}\right) \mid\left(\tilde{\omega}^{2}\right)_{1} u=\frac{2}{5} u\right\} .
\end{aligned}
$$

By a similar argument as in the $\tau$-twisted case, we can show that $M_{T}^{0}\left(\tau^{2}\right)$ and $W_{T}^{0}\left(\tau^{2}\right)$ are inequivalent simple $\tau^{2}$-twisted $M_{k}^{0}$-modules.

Take a nonzero $v$ in $T_{\chi_{j}^{\prime}}, j=1,2$. Then

$$
\left(\tilde{\omega}^{2}\right)_{1}(1 \otimes v)=\frac{1}{15}(1 \otimes v), \quad\left(\tilde{\omega}^{2}\right)_{1} v^{j}=\frac{2}{3} v^{j},
$$

where $v^{j}=h_{1}^{\prime}\left(-\frac{2}{3}\right) \otimes v-(-1)^{j} \sqrt{-3} h_{2}^{\prime}\left(-\frac{1}{3}\right)^{2} \otimes v$. Furthermore,

$$
K_{2}(1 \otimes v)=(-1)^{j} \frac{2}{9}(1 \otimes v), \quad K_{2} v^{j}=-(-1)^{j} \frac{52}{9} v^{j} .
$$

Hence $V_{L}^{j}\left(\tau^{2}\right) \cong\left(M_{T}^{j}\left(\tau^{2}\right) \otimes M_{t}^{j}\right) \oplus\left(W_{T}^{j}\left(\tau^{2}\right) \otimes W_{t}^{j}\right)$ as $\tau^{2}$-twisted $M_{k}^{0} \otimes M_{t}^{0}$-modules for $j=1,2$, where

$$
\begin{aligned}
& M_{T}^{j}\left(\tau^{2}\right)=\left\{u \in V_{L}^{j}\left(\tau^{2}\right) \mid\left(\tilde{\omega}^{2}\right)_{1} u=\frac{2}{3} u\right\}, \\
& W_{T}^{j}\left(\tau^{2}\right)=\left\{u \in V_{L}^{j}\left(\tau^{2}\right) \mid\left(\tilde{\omega}^{2}\right)_{1} u=\frac{1}{15} u\right\}, \quad j=1,2 .
\end{aligned}
$$

As in the $\tau$-twisted case, the $M_{T}^{j}\left(\tau^{2}\right), j=0,1,2$ are equivalent and the $W_{T}^{j}\left(\tau^{2}\right)$, $j=0,1,2$ are equivalent. Set $M_{T}\left(\tau^{2}\right)=M_{T}^{0}\left(\tau^{2}\right)$ and $W_{T}\left(\tau^{2}\right)=W_{T}^{0}\left(\tau^{2}\right)$. Then

$$
V_{L}^{j}\left(\tau^{2}\right) \cong\left(M_{T}\left(\tau^{2}\right) \otimes M_{t}^{j}\right) \oplus\left(W_{T}\left(\tau^{2}\right) \otimes W_{t}^{j}\right), \quad j=0,1,2,
$$

as $\tau^{2}$-twisted $M_{k}^{0} \otimes M_{t}^{0}$-modules. 
Remark 3.5. The weight-three vector $K$ was denoted by different symbols in previous papers, namely, $v_{t}, v^{3}$, and $q$ were used in [Dong et al. 2004], [Kitazume et al. 2003], and [Kitazume et al. 2000b], respectively. They are related as follows: $K=-2 \sqrt{2} v_{t}=-2 \sqrt{2} v^{3}=2 \sqrt{2} q$. Thus, in the proof of [Kitazume et al. 2003, Proposition 6.8] $\left(v^{3}\right)_{2}$ should act on the top level of $V_{L}^{j}(\tau)$ as a scalar multiple of $(-1)^{j} / 9 \sqrt{2}$ for $j=1,2$. Moreover, (6.46) of [Kitazume et al. 2003] and the equation for $V_{L}^{j}(\tau)$ on page 265 of [Dong et al. 2004] should be replaced with Equation (3-19). This correction does not affect the results in the latter paper. However, certain changes are necessary in [Kitazume et al. 2003] along with the correction.

Note that

$$
\begin{aligned}
& M_{T}\left(\tau^{i}\right)(\varepsilon)=\left\{u \in M_{T}\left(\tau^{i}\right) \mid \tau^{i} u=\xi^{\varepsilon} u\right\}, \\
& W_{T}\left(\tau^{i}\right)(\varepsilon)=\left\{u \in W_{T}\left(\tau^{i}\right) \mid \tau^{i} u=\xi^{\varepsilon} u\right\}
\end{aligned}
$$

for $i=1,2, \varepsilon=0,1,2$. Another notation was used in [Kitazume et al. 2003], namely,

$$
M_{T}\left(\tau^{i}\right)^{\varepsilon}=\bigoplus_{n \in 1 / 9+\varepsilon / 3+\mathbb{Z}}\left(M_{T}\left(\tau^{i}\right)\right)_{n}, \quad W_{T}\left(\tau^{i}\right)^{\varepsilon}=\bigoplus_{n \in 2 / 45+\varepsilon / 3+\mathbb{Z}}\left(W_{T}\left(\tau^{i}\right)\right)_{n},
$$

where $U_{n}$ denotes the eigenspace of $U$ with eigenvalue $n$ for $\left(\tilde{\omega}^{1}\right)_{1}$. The two sets of notation are related by

$$
M_{T}\left(\tau^{i}\right)^{\varepsilon}=M_{T}\left(\tau^{i}\right)(2 \varepsilon), \quad W_{T}\left(\tau^{i}\right)^{\varepsilon}=W_{T}\left(\tau^{i}\right)(2 \varepsilon-1) .
$$

Likewise,

$$
\left(V_{L}^{T_{\chi_{j}}}(\tau)\right)^{\varepsilon}=\bigoplus_{n \in 1 / 9+\varepsilon / 3+\mathbb{Z}}\left(V_{L}^{T_{\chi_{j}}}(\tau)\right)_{n}, \quad\left(V_{L}^{T_{\chi_{j}}}\left(\tau^{2}\right)\right)^{\varepsilon}=\bigoplus_{n \in 1 / 9+\varepsilon / 3+\mathbb{Z}}\left(V_{L}^{T_{\chi_{j}}}\left(\tau^{2}\right)\right)_{n}
$$

of [Kitazume et al. 2003, (7.16)] are denoted here by

$$
\left(V_{L}^{j}(\tau)\right)^{\varepsilon}=V_{L}^{j}(\tau)(2 \varepsilon), \quad\left(V_{L}^{j}\left(\tau^{2}\right)\right)^{\varepsilon}=V_{L}^{j}\left(\tau^{2}\right)(2 \varepsilon)
$$

for $j=0,1,2$ and $\varepsilon=0,1,2$, where $U_{n}$ is the eigenspace of $U$ with eigenvalue $n$ for $\omega_{1}$.

By (3-3), the minimal eigenvalues of $\left(\tilde{\omega}^{2}\right)_{1}$ on $M_{t}^{0}$ and $W_{t}^{0}$ are 0 and $2 / 5$, respectively, while those on $M_{t}^{j}$ and $W_{t}^{j}, j=1,2$, are $2 / 3$ and $1 / 15$, respectively. Hence it follows from (3-19) that

$$
\begin{aligned}
& \left(V_{L}^{0}(\tau)\right)^{\varepsilon} \cong\left(M_{T}(\tau)^{\varepsilon} \otimes M_{t}^{0}\right) \oplus\left(W_{T}(\tau)^{\varepsilon-1} \otimes W_{t}^{0}\right), \\
& \left(V_{L}^{j}(\tau)\right)^{\varepsilon} \cong\left(M_{T}(\tau)^{\varepsilon+1} \otimes M_{t}^{3-j}\right) \oplus\left(W_{T}(\tau)^{\varepsilon} \otimes W_{t}^{3-j}\right), \quad j=1,2,
\end{aligned}
$$


as $M^{0}$-modules for $\varepsilon=0,1,2$, where $M^{0}=M(0) \otimes M_{t}^{0}$. Similarly,

$$
\begin{aligned}
& \left(V_{L}^{0}\left(\tau^{2}\right)\right)^{\varepsilon} \cong\left(M_{T}\left(\tau^{2}\right)^{\varepsilon} \otimes M_{t}^{0}\right) \oplus\left(W_{T}\left(\tau^{2}\right)^{\varepsilon-1} \otimes W_{t}^{0}\right), \\
& \left(V_{L}^{j}\left(\tau^{2}\right)\right)^{\varepsilon} \cong\left(M_{T}\left(\tau^{2}\right)^{\varepsilon+1} \otimes M_{t}^{j}\right) \oplus\left(W_{T}\left(\tau^{2}\right)^{\varepsilon} \otimes W_{t}^{j}\right), \quad j=1,2,
\end{aligned}
$$

as $M^{0}$-modules for $\varepsilon=0,1,2$ (see [Kitazume et al. 2003, (7.17)]).

The following fusion rules of simple $M(0)$-modules will be necessary for the study of simple $V_{L}^{\tau}$-modules.

$$
\begin{aligned}
W(0) \times M_{k}^{c} & =W_{k}^{c}, \\
W(0) \times W_{k}^{c} & =M_{k}^{c}+W_{k}^{c}, \\
W(0) \times M(\varepsilon) & =W(\varepsilon), \\
W(0) \times W(\varepsilon) & =M(\varepsilon)+W(\varepsilon), \\
W(0) \times M_{T}\left(\tau^{i}\right)(\varepsilon) & =W_{T}\left(\tau^{i}\right)(\varepsilon), \\
W(0) \times W_{T}\left(\tau^{i}\right)(\varepsilon) & =M_{T}\left(\tau^{i}\right)(\varepsilon)+W_{T}\left(\tau^{i}\right)(\varepsilon)
\end{aligned}
$$

for $i=1,2$ and $\varepsilon=0,1,2$. In fact, the first four fusion rules, that is, the fusion rules among simple $M(0)$-modules appearing in untwisted simple $V_{L}$-modules, can be found in [Tanabe 2005]. The last two fusion rules involve simple $M(0)$-modules that appear in $\tau^{i}$-twisted simple $V_{L}$-modules. Their proofs can be found in the Appendix.

Fusion rules possess certain symmetries. Let $M^{i}, i=1,2,3$ be modules for a vertex operator algebra $V$. Then by [Frenkel et al. 1993, Propositions 5.4.7 and 5.5.2]

$$
\operatorname{dim} I_{V}\left(\begin{array}{c}
M^{3} \\
M^{1} M^{2}
\end{array}\right)=\operatorname{dim} I_{V}\left(\begin{array}{c}
M^{3} \\
M^{2} M^{1}
\end{array}\right)=\operatorname{dim} I_{V}\left(\begin{array}{c}
\left(M^{2}\right)^{\prime} \\
M^{1}\left(M^{3}\right)^{\prime}
\end{array}\right)
$$

where $\left(M^{i}\right)^{\prime}$ is the contragredient module of $M^{i}$. Recall that the contragredient module $\left(U^{\prime}, Y_{U^{\prime}}\right)$ of a $V$-module $\left(U, Y_{U}\right)$ is defined as follows. As a vector space $U^{\prime}=\bigoplus_{n}\left(U_{n}\right)^{*}$ is the restricted dual of $U$ and $Y_{U^{\prime}}(\cdot, z)$ is determined by

$$
\left\langle Y_{U^{\prime}}(a, z) v, u\right\rangle=\left\langle v, Y_{U}\left(e^{z L(1)}\left(-z^{-2}\right)^{L(0)} a, z^{-1}\right) u\right\rangle
$$

for $a \in V, u \in U$, and $v \in U^{\prime}$.

In our case $M(0)$ is generated by the Virasoro element $\tilde{\omega}^{1}$ and the weight-three vector $J$. Moreover, $\left\langle L^{1}(0) v, u\right\rangle=\left\langle v, L^{1}(0) u\right\rangle$ and $\langle J(0) v, u\rangle=-\langle v, J(0) u\rangle$. Since the 20 simple $M(0)$-modules are distinguished by the action of $L^{1}(0)$ and $J(0)$ on their top levels, we know from [Dong et al. 2004, Tables 1, 3, and 4] that 
the contragredient modules of the simple $M(0)$-modules are as follows.

$$
\begin{gathered}
M(\varepsilon)^{\prime} \cong M(2 \varepsilon), \quad W(\varepsilon)^{\prime} \cong W(2 \varepsilon), \quad \varepsilon=0,1,2, \\
\left(M_{k}^{c}\right)^{\prime} \cong M_{k}^{c}, \quad\left(W_{k}^{c}\right)^{\prime} \cong W_{k}^{c}, \\
M_{T}(\tau)(\varepsilon)^{\prime} \cong M_{T}\left(\tau^{2}\right)(\varepsilon), \quad W_{T}(\tau)(\varepsilon)^{\prime} \cong W_{T}\left(\tau^{2}\right)(\varepsilon), \quad \varepsilon=0,1,2
\end{gathered}
$$

(see also [Dong et al. 1998a, Lemma 3.7] and [Tanabe 2005, Section 4.2]).

\section{Structure of simple modules}

Recall that $V_{L}^{\tau}=V_{L}(0)=M^{0} \oplus W^{0}$ with $M^{0}=M(0) \otimes M_{t}^{0}$ and $W^{0}=W(0) \otimes W_{t}^{0}$. In this section we study the structure of the 30 known simple $V_{L}^{\tau}$-modules listed in Lemma 3.2. We discuss decompositions of these simple modules as modules for $M^{0}$. Those decompositions have been obtained in [Kitazume et al. 2003]. We review them briefly. (Some corrections are needed in that paper; see Remark 3.5.)

A vector in a $V_{L}^{\tau}$-module is said to be of weight $h$ if it is an eigenvector for $L(0)=\omega_{1}$ with eigenvalue $h$. We calculate the action of $\left(\tilde{\omega}^{1}\right)_{1},\left(\tilde{\omega}^{2}\right)_{1}, J_{2}, K_{2}, P_{1}$, $\left(J_{1} P\right)_{2}$, and $\left(K_{1} P\right)_{2}$ on the top levels of the 30 known simple $V_{L}^{\tau}$-modules. Recall that the top level of a module means the homogeneous subspace of the module of smallest weight. The calculation is accomplished directly from the definition of untwisted or twisted vertex operators associated with the lattice $L$ and the automorphisms $\tau$ and $\tau^{2}$ (see [Dong and Lepowsky 1996; Frenkel et al. 1988; Lepowsky and Li 2004]). The results in this section will be used to determine the Zhu algebra $A\left(V_{L}^{\tau}\right)$ of $V_{L}^{\tau}$ in Section 5.

The vectors $J_{1} P$ and $K_{1} P$ are of weight 3 . Their precise form in terms of the lattice vertex operator algebra $V_{L}$ is as follows.

$$
\begin{aligned}
J_{1} P= & 2 \beta_{1}(-1)^{3}+3 \beta_{1}(-1)^{2} \beta_{2}(-1)-3 \beta_{1}(-1) \beta_{2}(-1)^{2}-2 \beta_{2}(-1)^{3} \\
& \quad-4\left(\left(\beta_{2}-\beta_{0}\right)(-1) x\left(\alpha_{1}\right)+\left(\beta_{0}-\beta_{1}\right)(-1) x\left(\alpha_{2}\right)+\left(\beta_{1}-\beta_{2}\right)(-1) x\left(\alpha_{0}\right)\right) \\
= & \frac{13}{9}\left(2 \beta_{1}(-1)^{3}+3 \beta_{1}(-1)^{2} \beta_{2}(-1)-3 \beta_{1}(-1) \beta_{2}(-1)^{2}-2 \beta_{2}(-1)^{3}\right)-4 K, \\
K_{1} P= & 3\left(\beta_{1}(-2) \beta_{2}(-1)-\beta_{2}(-2) \beta_{1}(-1)\right) \\
& \quad-\left(\left(\beta_{2}-\beta_{0}\right)(-1) y\left(\alpha_{1}\right)+\left(\beta_{0}-\beta_{1}\right)(-1) y\left(\alpha_{2}\right)+\left(\beta_{1}-\beta_{2}\right)(-1) y\left(\alpha_{0}\right)\right) \\
= & \frac{7}{2}\left(\beta_{1}(-2) \beta_{2}(-1)-\beta_{2}(-2) \beta_{1}(-1)\right)+J .
\end{aligned}
$$

The simple module $V_{L}(0) . \quad V_{L}(0)=M^{0} \oplus W^{0}$ as $M^{0}$-modules. The top level of $V_{L}(0)$ is $\mathbb{C} 1$. By a property of the vacuum vector, all of $\left(\tilde{\omega}^{1}\right)_{1},\left(\tilde{\omega}^{2}\right)_{1}, J_{2}, K_{2}, P_{1}$, $\left(J_{1} P\right)_{2}$, and $\left(K_{1} P\right)_{2}$ act as 0 on $\mathbb{C} \mathbf{1}$.

The simple module $V_{L}(\varepsilon), \varepsilon=1,2$. By (3-16), we have

$$
V_{L}(\varepsilon) \cong\left(M(\varepsilon) \otimes M_{t}^{0}\right) \oplus\left(W(\varepsilon) \otimes W_{t}^{0}\right)
$$


as $M^{0}$-modules for $\varepsilon=1,2$. The top level of $V_{L}(\varepsilon)$ is $\mathbb{C} \mathbf{v}^{2, \varepsilon}$, where $\mathbf{v}^{2, \varepsilon}=\alpha_{1}(-1)-$ $\xi^{\varepsilon} \alpha_{2}(-1) \in W(\varepsilon) \otimes W_{t}^{0}$. We have

$$
\left(\tilde{\omega}^{1}\right)_{1} \mathbf{v}^{2, \varepsilon}=\frac{3}{5} \mathbf{v}^{2, \varepsilon}, \quad\left(\tilde{\omega}^{2}\right)_{1} \mathbf{v}^{2, \varepsilon}=\frac{2}{5} \mathbf{v}^{2, \varepsilon}, \quad J_{2} \mathbf{v}^{2, \varepsilon}=-(-1)^{\varepsilon} 2 \sqrt{-3} \mathbf{v}^{2, \varepsilon},
$$

$K_{2} \mathbf{v}^{2, \varepsilon}=0, \quad P_{1} \mathbf{v}^{2, \varepsilon}=0, \quad\left(J_{1} P\right)_{2} \mathbf{v}^{2, \varepsilon}=0, \quad\left(K_{1} P\right)_{2} \mathbf{v}^{2, \varepsilon}=(-1)^{\varepsilon} 12 \sqrt{-3} \mathbf{v}^{2, \varepsilon}$.

The simple module $\boldsymbol{V}_{\boldsymbol{L}^{(0, j)}}(\mathbf{0}), \boldsymbol{j}=1,2$. For $j=1,2$, (3-4) implies that $V_{L^{(0, j)}}$ is a direct sum of simple $M^{0}$-modules of the form $A \otimes B$, where $A$ is a simple $M(0)$ module and $B$ is a simple $M_{t}^{0}$-module isomorphic to $M_{t}^{j}$ or $W_{t}^{j}$. For convenience, set $U^{j}(\varepsilon)=V_{L^{(0, j)}}(\varepsilon), j=1,2, \varepsilon=0,1,2$. Let

$$
\mathbf{v}^{3, j}=e^{(-1)^{j}\left(\beta_{1}-\beta_{2}\right) / 3}+e^{(-1)^{j}\left(\beta_{2}-\beta_{0}\right) / 3}+e^{(-1)^{j}\left(\beta_{0}-\beta_{1}\right) / 3} .
$$

Then $\mathbf{v}^{3, j} \in U^{j}(0)$. Moreover, $\left(\omega^{1}\right)_{1} \mathbf{v}^{3, j}=\left(\omega^{2}\right)_{1} \mathbf{v}^{3, j}=0$ and $\left(\tilde{\omega}^{2}\right)_{1} \mathbf{v}^{3, j}=(2 / 3) \mathbf{v}^{3, j}$. Hence $\mathbf{v}^{3, j} \in M_{t}^{j}$ and $U^{j}(0)$ contains an $M_{t}^{0}$-submodule isomorphic to $M_{t}^{j}$. By the fusion rule $M_{t}^{j} \times W_{t}^{0}=W_{t}^{j}$ of $M_{t}^{0}$-modules and [Dong and Lepowsky 1996, Proposition 11.9], $U^{j}(0)$ contains an $M_{t}^{0}$-submodule isomorphic to $W_{t}^{j}$ also. Thus $U^{j}(0)$ contains simple $M^{0}$-submodules of the form $A \otimes M_{t}^{j}$ and $A^{\prime} \otimes W_{t}^{j}$ for some simple $M(0)$-modules $A$ and $A^{\prime}$.

The minimal weight of $V_{L^{(0, j)}}$ is $2 / 3$. Its weight subspace is of dimension 3 and spanned by $e^{(-1)^{j}\left(\beta_{1}-\beta_{2}\right) / 3}, e^{(-1)^{j}\left(\beta_{2}-\beta_{0}\right) / 3}$, and $e^{(-1)^{j}\left(\beta_{0}-\beta_{1}\right) / 3}$. Thus the weight- $2 / 3$ subspace of $U^{j}(0)$ is $\mathbb{C} \mathbf{v}^{3, j}$. Since $\left(\tilde{\omega}^{1}\right)_{1} \mathbf{v}^{3, j}=0$ and since only $M(0)$ is the simple $M(0)$-module whose minimal weight (= eigenvalue of $\left.\left(\tilde{\omega}^{1}\right)_{1}\right)$ is 0 by [Dong et al. 2004], we conclude that $U^{j}(0)$ contains a simple $M^{0}$-submodule isomorphic to $M(0) \otimes M_{t}^{j}$.

The minimal eigenvalue of $\left(\tilde{\omega}^{2}\right)_{1}$ in $W_{t}^{j}$ is $1 / 15$. Thus the eigenvalues of $\left(\tilde{\omega}^{1}\right)_{1}$ on $A^{\prime}$ must be of the form $3 / 5+n, n \in \mathbb{Z}$. By [Dong et al. 2004], only $W(0)$, $W(1), W(2)$ are the simple $M(0)$-modules whose weights are of this form. The minimal weight of these simple modules are $8 / 5,3 / 5$ and $3 / 5$, respectively. Since the weight- $2 / 3$ subspace of $U^{j}(0)$ is one dimensional, we see that $U^{j}(0)$ contains a simple $M^{0}$-submodule isomorphic to $W(0) \otimes W_{t}^{j}$.

From the fusion rules for $M_{t}^{0}$-modules, we obtain the fusion rules

$$
\begin{aligned}
& \left(M(\varepsilon) \otimes M_{t}^{0}\right) \times\left(M(0) \otimes M_{t}^{j}\right)=M(\varepsilon) \otimes M_{t}^{j}, \\
& \left(W(\varepsilon) \otimes W_{t}^{0}\right) \times\left(M(0) \otimes M_{t}^{j}\right)=W(\varepsilon) \otimes W_{t}^{j}
\end{aligned}
$$

for $M^{0}$-modules. Hence $U^{j}(\varepsilon) \cong\left(M(\varepsilon) \otimes M_{t}^{j}\right) \oplus\left(W(\varepsilon) \otimes W_{t}^{j}\right)$ for $j=1,2$ and $\varepsilon=$ $0,1,2$ by (3-4) and (3-16). In particular, $V_{L^{(0, j)}}(0) \cong\left(M(0) \otimes M_{t}^{j}\right) \oplus\left(W(0) \otimes W_{t}^{j}\right)$ as $M^{0}$-modules, $j=1,2$. The top level of $V_{L^{(0, j)}}(0)$ is $\mathbb{C} \mathbf{v}^{3, j} \subset M(0) \otimes M_{t}^{j}$. We have 


$$
\begin{gathered}
\left(\tilde{\omega}^{1}\right)_{1} \mathbf{v}^{3, j}=0, \quad\left(\tilde{\omega}^{2}\right)_{1} \mathbf{v}^{3, j}=\frac{2}{3} \mathbf{v}^{3, j}, \quad J_{2} \mathbf{v}^{3, j}=0, \quad K_{2} \mathbf{v}^{3, j}=-(-1)^{j} \frac{52}{9} \mathbf{v}^{3, j}, \\
P_{1} \mathbf{v}^{3, j}=0, \quad\left(J_{1} P\right)_{2} \mathbf{v}^{3, j}=0, \quad\left(K_{1} P\right)_{2} \mathbf{v}^{3, j}=0 .
\end{gathered}
$$

The simple module $V_{L^{(0, j)}}(\varepsilon), j=1,2, \varepsilon=1,2$. We have shown above that $V_{L^{(0, j)}}(\varepsilon) \cong\left(M(\varepsilon) \otimes M_{t}^{j}\right) \oplus\left(W(\varepsilon) \otimes W_{t}^{j}\right)$ as $M^{0}$-modules, $j=1,2, \varepsilon=1,2$. The top level of $V_{L^{(0, j)}}(\varepsilon)$ is $\mathbb{C} \mathbf{v}^{4, j, \varepsilon}$, where

$$
\mathbf{v}^{4, j, \varepsilon}=e^{(-1)^{j}\left(\beta_{1}-\beta_{2}\right) / 3}+\xi^{2 \varepsilon} e^{(-1)^{j}\left(\beta_{2}-\beta_{0}\right) / 3}+\xi^{\varepsilon} e^{(-1)^{j}\left(\beta_{0}-\beta_{1}\right) / 3} \in W(\varepsilon) \otimes W_{t}^{j} .
$$

We have

$$
\begin{gathered}
\left(\tilde{\omega}^{1}\right)_{1} \mathbf{v}^{4, j, \varepsilon}=\frac{3}{5} \mathbf{v}^{4, j, \varepsilon}, \quad\left(\tilde{\omega}^{2}\right)_{1} \mathbf{v}^{4, j, \varepsilon}=\frac{1}{15} \mathbf{v}^{4, j, \varepsilon}, \quad J_{2} \mathbf{v}^{4, j, \varepsilon}=-(-1)^{\varepsilon} 2 \sqrt{-3} \mathbf{v}^{4, j, \varepsilon}, \\
K_{2} \mathbf{v}^{4, j, \varepsilon}=(-1)^{j} \frac{2}{9} \mathbf{v}^{4, j, \varepsilon}, \quad P_{1} \mathbf{v}^{4, j, \varepsilon}=-(-1)^{j+\varepsilon} \sqrt{-3} \mathbf{v}^{4, j, \varepsilon}, \\
\left(J_{1} P\right)_{2} \mathbf{v}^{4, j, \varepsilon}=-(-1)^{j} 24 \mathbf{v}^{4, j, \varepsilon}, \quad\left(K_{1} P\right)_{2} \mathbf{v}^{4, j, \varepsilon}=-(-1)^{\varepsilon} 2 \sqrt{-3} \mathbf{v}^{4, j, \varepsilon} .
\end{gathered}
$$

The simple module $\boldsymbol{V}_{\boldsymbol{L}^{(\boldsymbol{c}, 0)}}$. By (3-4), $V_{L^{(c, 0)}} \cong\left(M_{k}^{c} \otimes M_{t}^{0}\right) \oplus\left(W_{k}^{c} \otimes W_{t}^{0}\right)$ as $M^{0}$ modules. The top level of $V_{L^{(c, 0)}}$ is of dimension 2 with basis $\left\{\mathbf{v}^{5,1}, \mathbf{v}^{5,2}\right\}$, where $\mathbf{v}^{5,1}=e^{\beta_{1} / 2}-e^{-\beta_{1} / 2} \in M_{k}^{c} \otimes M_{t}^{0}, \mathbf{v}^{5,2}=e^{\beta_{1} / 2}+e^{-\beta_{1} / 2} \in W_{k}^{c} \otimes W_{t}^{0}$. We have

$$
\begin{gathered}
\left(\tilde{\omega}^{1}\right)_{1} \mathbf{v}^{5,1}=\frac{1}{2} \mathbf{v}^{5,1}, \quad\left(\tilde{\omega}^{1}\right)_{1} \mathbf{v}^{5,2}=\frac{1}{10} \mathbf{v}^{5,2}, \quad\left(\tilde{\omega}^{2}\right)_{1} \mathbf{v}^{5,1}=0, \quad\left(\tilde{\omega}^{2}\right)_{1} \mathbf{v}^{5,2}=\frac{2}{5} \mathbf{v}^{5,2}, \\
J_{2} \mathbf{v}^{5, j}=0, \quad K_{2} \mathbf{v}^{5, j}=0, j=1,2, \quad P_{1} \mathbf{v}^{5,1}=-\mathbf{v}^{5,2}, \quad P_{1} \mathbf{v}^{5,2}=\mathbf{v}^{5,1} \\
\left(J_{1} P\right)_{2} \mathbf{v}^{5, j}=0, \quad\left(K_{1} P\right)_{2} \mathbf{v}^{5, j}=0, j=1,2 .
\end{gathered}
$$

The simple module $\boldsymbol{V}_{\boldsymbol{L}^{(c, j)}}, \boldsymbol{j}=\mathbf{1}, \mathbf{2}$. By (3-4), we have the isomorphism $V_{L^{(c, j)}} \cong$ $\left(M_{k}^{c} \otimes M_{t}^{j}\right) \oplus\left(W_{k}^{c} \otimes W_{t}^{j}\right)$ as $M^{0}$-modules, $j=1$, 2. The top level of $V_{L^{(c, j)}}$ is $\mathbb{C} \mathbf{v}^{6, j}$, where $\mathbf{v}^{6, j}=e^{-(-1)^{j}\left(\beta_{2}-\beta_{0}\right) / 6} \in W_{k}^{c} \otimes W_{t}^{j}$. We have

$$
\begin{gathered}
\left(\tilde{\omega}^{1}\right)_{1} \mathbf{v}^{6, j}=\frac{1}{10} \mathbf{v}^{6, j}, \quad\left(\tilde{\omega}^{2}\right)_{1} \mathbf{v}^{6, j}=\frac{1}{15} \mathbf{v}^{6, j}, \quad J_{2} \mathbf{v}^{6, j}=0, \quad K_{2} \mathbf{v}^{6, j}=(-1)^{j} \frac{2}{9} \mathbf{v}^{6, j}, \\
P_{1} \mathbf{v}^{6, j}=0, \quad\left(J_{1} P\right)_{2} \mathbf{v}^{6, j}=(-1)^{j} 2 \mathbf{v}^{6, j}, \quad\left(K_{1} P\right)_{2} \mathbf{v}^{6, j}=0 .
\end{gathered}
$$

The simple module $\boldsymbol{V}_{\boldsymbol{L}}^{\mathbf{0}}(\boldsymbol{\tau})(\mathbf{0})$. By (3-23), we have the isomorphism $V_{L}^{0}(\tau)(0) \cong$ $\left(M_{T}(\tau)(0) \otimes M_{t}^{0}\right) \oplus\left(W_{T}(\tau)(0) \otimes W_{t}^{0}\right)$ as $M^{0}$-modules. The top level of $V_{L}^{0}(\tau)(0)$ is $\mathbb{C} \mathbf{v}^{7}$, where $\mathbf{v}^{7}=1 \otimes v \in M_{T}(\tau)(0) \otimes M_{t}^{0}$ and $0 \neq v \in T_{\chi_{0}}$. We have

$$
\begin{gathered}
\left(\tilde{\omega}^{1}\right)_{1} \mathbf{v}^{7}=\frac{1}{9} \mathbf{v}^{7}, \quad\left(\tilde{\omega}^{2}\right)_{1} \mathbf{v}^{7}=0, \quad J_{2} \mathbf{v}^{7}=\frac{14}{81} \sqrt{-3} \mathbf{v}^{7}, \quad K_{2} \mathbf{v}^{7}=0, \\
P_{1} \mathbf{v}^{7}=0, \quad\left(J_{1} P\right)_{2} \mathbf{v}^{7}=0, \quad\left(K_{1} P\right)_{2} \mathbf{v}^{7}=0 .
\end{gathered}
$$

The simple module $\boldsymbol{V}_{\boldsymbol{L}}^{\mathbf{0}}(\boldsymbol{\tau})(1)$. By (3-23), we have the isomorphism $V_{L}^{0}(\tau)(1) \cong$ $\left(M_{T}(\tau)(1) \otimes M_{t}^{0}\right) \oplus\left(W_{T}(\tau)(1) \otimes W_{t}^{0}\right)$ as $M^{0}$-modules. The top level of $V_{L}^{0}(\tau)(1)$ is of dimension 2 with basis $\left\{\mathbf{v}^{8,1}, \mathbf{v}^{8,2}\right\}$, where

$$
\begin{aligned}
& \mathbf{v}^{8,1}=h_{2}(-1 / 3)^{2} \otimes v \in M_{T}(\tau)(1) \otimes M_{t}^{0}, \\
& \mathbf{v}^{8,2}=h_{1}(-2 / 3) \otimes v \in W_{T}(\tau)(1) \otimes W_{t}^{0}
\end{aligned}
$$


and $0 \neq v \in T_{\chi_{0}}$. We have

$$
\begin{gathered}
\left(\tilde{\omega}^{1}\right)_{1} \mathbf{v}^{8,1}=\left(\frac{1}{9}+\frac{2}{3}\right) \mathbf{v}^{8,1}, \quad\left(\tilde{\omega}^{2}\right)_{1} \mathbf{v}^{8,1}=0, \quad J_{2} \mathbf{v}^{8,1}=-\frac{238}{81} \sqrt{-3} \mathbf{v}^{8,1}, \quad K_{2} \mathbf{v}^{8,1}=0 \\
P_{1} \mathbf{v}^{8,1}=-\frac{4}{3} \mathbf{v}^{8,2}, \quad\left(J_{1} P\right)_{2} \mathbf{v}^{8,1}=\frac{104}{9} \sqrt{-3} \mathbf{v}^{8,2}, \quad\left(K_{1} P\right)_{2} \mathbf{v}^{8,1}=0 \\
\left(\tilde{\omega}^{1}\right)_{1} \mathbf{v}^{8,2}=\left(\frac{2}{45}+\frac{1}{3}\right) \mathbf{v}^{8,2}, \quad\left(\tilde{\omega}^{2}\right)_{1} \mathbf{v}^{8,2}=\frac{2}{5} \mathbf{v}^{8,2}, \quad J_{2} \mathbf{v}^{8,2}=-\frac{22}{81} \sqrt{-3} \mathbf{v}^{8,2}, \quad K_{2} \mathbf{v}^{8,2}=0 \\
P_{1} \mathbf{v}^{8,2}=2 \mathbf{v}^{8,1},\left(J_{1} P\right)_{2} \mathbf{v}^{8,2}=-\frac{52}{3} \sqrt{-3} \mathbf{v}^{8,1},\left(K_{1} P\right)_{2} \mathbf{v}^{8,2}=-\frac{20}{3} \sqrt{-3} \mathbf{v}^{8,2} .
\end{gathered}
$$

The simple module $\boldsymbol{V}_{\boldsymbol{L}}^{\mathbf{0}}(\boldsymbol{\tau})(2)$. By (3-23), we have the isomorphism $V_{L}^{0}(\tau)(2) \cong$ $\left(M_{T}(\tau)(2) \otimes M_{t}^{0}\right) \oplus\left(W_{T}(\tau)(2) \otimes W_{t}^{0}\right)$ as $M^{0}$-modules. The top level of $V_{L}^{0}(\tau)(2)$ is $\mathbb{C} \mathbf{v}^{9}$, where $\mathbf{v}^{9}=h_{2}(-1 / 3) \otimes v \in W_{T}(\tau)(2) \otimes W_{t}^{0}$ and $0 \neq v \in T_{\chi_{0}}$. We have

$$
\begin{gathered}
\left(\tilde{\omega}^{1}\right)_{1} \mathbf{v}^{9}=\frac{2}{45} \mathbf{v}^{9}, \quad\left(\tilde{\omega}^{2}\right)_{1} \mathbf{v}^{9}=\frac{2}{5} \mathbf{v}^{9}, \quad J_{2} \mathbf{v}^{9}=-\frac{4}{81} \sqrt{-3} \mathbf{v}^{9}, \quad K_{2} \mathbf{v}^{9}=0, \\
P_{1} \mathbf{v}^{9}=0, \quad\left(J_{1} P\right)_{2} \mathbf{v}^{9}=0, \quad\left(K_{1} P\right)_{2} \mathbf{v}^{9}=\frac{4}{3} \sqrt{-3} \mathbf{v}^{9} .
\end{gathered}
$$

The simple module $V_{L}^{j}(\tau)(0), j=1,2$. By (3-23), we have the isomorphism $V_{L}^{j}(\tau)(0) \cong\left(M_{T}(\tau)(2) \otimes M_{t}^{3-j}\right) \oplus\left(W_{T}(\tau)(2) \otimes W_{t}^{3-j}\right)$ as $M^{0}$-modules for $j=1,2$. The top level of $V_{L}^{j}(\tau)(0)$ is $\mathbb{C} \mathbf{v}^{10, j}$, where $\mathbf{v}^{10, j}=1 \otimes v \in W_{T}(\tau)(2) \otimes W_{t}^{3-j}$ and $0 \neq v \in T_{\chi_{j}}$. We have

$$
\begin{gathered}
\left(\tilde{\omega}^{1}\right)_{1} \mathbf{v}^{10, j}=\frac{2}{45} \mathbf{v}^{10, j}, \quad\left(\tilde{\omega}^{2}\right)_{1} \mathbf{v}^{10, j}=\frac{1}{15} \mathbf{v}^{10, j}, \quad J_{2} \mathbf{v}^{10, j}=-\frac{4}{81} \sqrt{-3} \mathbf{v}^{10, j}, \\
K_{2} \mathbf{v}^{10, j}=-(-1)^{j} \frac{2}{9} \mathbf{v}^{10, j}, \quad P_{1} \mathbf{v}^{10, j}=(-1)^{j} \frac{1}{9} \sqrt{-3} \mathbf{v}^{10, j}, \\
\left(J_{1} P\right)_{2} \mathbf{v}^{10, j}=(-1)^{j} \frac{8}{9} \mathbf{v}^{10, j}, \quad\left(K_{1} P\right)_{2} \mathbf{v}^{10, j}=-\frac{2}{9} \sqrt{-3} \mathbf{v}^{10, j} .
\end{gathered}
$$

The simple module $V_{L}^{j}(\tau)(1), j=1,2$. By (3-23), we have the isomorphism $V_{L}^{j}(\tau)(1) \cong\left(M_{T}(\tau)(0) \otimes M_{t}^{3-j}\right) \oplus\left(W_{T}(\tau)(0) \otimes W_{t}^{3-j}\right)$ as $M^{0}$-modules for $j=1,2$. The top level of $V_{L}^{j}(\tau)(1)$ is of dimension 2 with basis $\left\{\mathbf{v}^{11, j, 1}, \mathbf{v}^{11, j, 2}\right\}$, where

$$
\begin{aligned}
& \mathbf{v}^{11, j, 1}=h_{1}(-2 / 3) \otimes v-(-1)^{j} \sqrt{-3} h_{2}(-1 / 3)^{2} \otimes v \in M_{T}(\tau)(0) \otimes M_{t}^{3-j}, \\
& \mathbf{v}^{11, j, 2}=2 h_{1}(-2 / 3) \otimes v+(-1)^{j} \sqrt{-3} h_{2}(-1 / 3)^{2} \otimes v \in W_{T}(\tau)(0) \otimes W_{t}^{3-j}
\end{aligned}
$$

and $0 \neq v \in T_{\chi_{j}}$. We have

$$
\begin{gathered}
\left(\tilde{\omega}^{1}\right)_{1} \mathbf{v}^{11, j, 1}=\frac{1}{9} \mathbf{v}^{11, j, 1}, \quad\left(\tilde{\omega}^{2}\right)_{1} \mathbf{v}^{11, j, 1}=\frac{2}{3} \mathbf{v}^{11, j, 1}, \quad J_{2} \mathbf{v}^{11, j, 1}=\frac{14}{81} \sqrt{-3} \mathbf{v}^{11, j, 1}, \\
K_{2} \mathbf{v}^{11, j, 1}=(-1)^{j} \frac{52}{9} \mathbf{v}^{11, j, 1}, \quad P_{1} \mathbf{v}^{11, j, 1}=-(-1)^{j} \frac{4}{9} \sqrt{-3} \mathbf{v}^{11, j, 2} \\
\left(J_{1} P\right)_{2} \mathbf{v}^{11, j, 1}=(-1)^{j} \frac{52}{9} \mathbf{v}^{11, j, 2}, \quad\left(K_{1} P\right)_{2} \mathbf{v}^{11, j, 1}=-\frac{28}{9} \sqrt{-3} \mathbf{v}^{11, j, 2} \\
\left(\tilde{\omega}^{1}\right)_{1} \mathbf{v}^{11, j, 2}=\left(\frac{2}{45}+\frac{2}{3}\right) \mathbf{v}^{11, j, 2}, \quad\left(\tilde{\omega}^{2}\right)_{1} \mathbf{v}^{11, j, 2}=\frac{1}{15} \mathbf{v}^{11, j, 2}
\end{gathered}
$$




$$
\begin{gathered}
J_{2} \mathbf{v}^{11, j, 2}=\frac{176}{81} \sqrt{-3} \mathbf{v}^{11, j, 2}, \quad K_{2} \mathbf{v}^{11, j, 2}=-(-1)^{j} \frac{2}{9} \mathbf{v}^{11, j, 2} \\
P_{1} \mathbf{v}^{11, j, 2}=-(-1)^{j} \frac{8}{9} \sqrt{-3} \mathbf{v}^{11, j, 1}+(-1)^{j} \frac{5}{9} \sqrt{-3} \mathbf{v}^{11, j, 2} \\
\left(J_{1} P\right)_{2} \mathbf{v}^{11, j, 2}=(-1)^{j} \frac{104}{9} \mathbf{v}^{11, j, 1}-(-1)^{j} \frac{200}{9} \mathbf{v}^{11, j, 2} \\
\left(K_{1} P\right)_{2} \mathbf{v}^{11, j, 2}=-\frac{56}{9} \sqrt{-3} \mathbf{v}^{11, j, 1}-\frac{10}{9} \sqrt{-3} \mathbf{v}^{11, j, 2}
\end{gathered}
$$

The simple module $V_{L}^{j}(\tau)(2), j=1,2$. By (3-23), we have the isomorphism $V_{L}^{j}(\tau)(2) \cong\left(M_{T}(\tau)(1) \otimes M_{t}^{3-j}\right) \oplus\left(W_{T}(\tau)(1) \otimes W_{t}^{3-j}\right)$ as $M^{0}$-modules for $j=1,2$. The top level of $V_{L}^{j}(\tau)(2)$ is $\mathbb{C} \mathbf{v}^{12, j}$, where $\mathbf{v}^{12, j}=h_{2}(-1 / 3) \otimes v \in W_{T}(\tau)(1) \otimes$ $W_{t}^{3-j}$ and $0 \neq v \in T_{\chi_{j}}$. We have

$$
\begin{gathered}
\left(\tilde{\omega}^{1}\right)_{1} \mathbf{v}^{12, j}=\left(\frac{2}{45}+\frac{1}{3}\right) \mathbf{v}^{12, j}, \quad\left(\tilde{\omega}^{2}\right)_{1} \mathbf{v}^{12, j}=\frac{1}{15} \mathbf{v}^{12, j}, \quad J_{2} \mathbf{v}^{12, j}=-\frac{22}{81} \sqrt{-3} \mathbf{v}^{12, j}, \\
K_{2} \mathbf{v}^{12, j}=-(-1)^{j} \frac{2}{9} \mathbf{v}^{12, j}, \quad P_{1} \mathbf{v}^{12, j}=-(-1)^{j} \frac{5}{9} \sqrt{-3} \mathbf{v}^{12, j} \\
\left(J_{1} P\right)_{2} \mathbf{v}^{12, j}=(-1)^{j} \frac{8}{9} \mathbf{v}^{12, j}, \quad\left(K_{1} P\right)_{2} \mathbf{v}^{12, j}=\frac{10}{9} \sqrt{-3} \mathbf{v}^{12, j}
\end{gathered}
$$

The simple module $\boldsymbol{V}_{\boldsymbol{L}}^{\mathbf{0}}\left(\boldsymbol{\tau}^{\mathbf{2}}\right)(\mathbf{0})$. By (3-24), we have the isomorphism $V_{L}^{0}\left(\tau^{2}\right)(0) \cong$ $\left(M_{T}\left(\tau^{2}\right)(0) \otimes M_{t}^{0}\right) \oplus\left(W_{T}\left(\tau^{2}\right)(0) \otimes W_{t}^{0}\right)$ as $M^{0}$-modules. The top level of $V_{L}^{0}\left(\tau^{2}\right)(0)$ is $\mathbb{C} \mathbf{v}^{13}$, where $\mathbf{v}^{13}=1 \otimes v \in M_{T}\left(\tau^{2}\right)(0) \otimes M_{t}^{0}$ and $0 \neq v \in T_{\chi_{0}^{\prime}}$. We have

$$
\begin{gathered}
\left(\tilde{\omega}^{1}\right)_{1} \mathbf{v}^{13}=\frac{1}{9} \mathbf{v}^{13}, \quad\left(\tilde{\omega}^{2}\right)_{1} \mathbf{v}^{13}=0, \quad J_{2} \mathbf{v}^{13}=-\frac{14}{81} \sqrt{-3} \mathbf{v}^{13}, \quad K_{2} \mathbf{v}^{13}=0, \\
P_{1} \mathbf{v}^{13}=0, \quad\left(J_{1} P\right)_{2} \mathbf{v}^{13}=0, \quad\left(K_{1} P\right)_{2} \mathbf{v}^{13}=0 .
\end{gathered}
$$

The simple module $\boldsymbol{V}_{\boldsymbol{L}}^{\mathbf{0}}\left(\boldsymbol{\tau}^{\mathbf{2}}\right)(\mathbf{1})$. By (3-24), we have the isomorphism $V_{L}^{0}\left(\tau^{2}\right)(1) \cong$ $\left(M_{T}\left(\tau^{2}\right)(1) \otimes M_{t}^{0}\right) \oplus\left(W_{T}\left(\tau^{2}\right)(1) \otimes W_{t}^{0}\right)$ as $M^{0}$-modules. The top level of $V_{L}^{0}\left(\tau^{2}\right)(1)$ is of dimension 2 with basis $\left\{\mathbf{v}^{14,1}, \mathbf{v}^{14,2}\right\}$, where

$$
\begin{aligned}
& \mathbf{v}^{14,1}=h_{2}^{\prime}(-1 / 3)^{2} \otimes v \in M_{T}\left(\tau^{2}\right)(1) \otimes M_{t}^{0}, \\
& \mathbf{v}^{14,2}=h_{1}^{\prime}(-2 / 3) \otimes v \in W_{T}\left(\tau^{2}\right)(1) \otimes W_{t}^{0},
\end{aligned}
$$

and $0 \neq v \in T_{\chi_{0}^{\prime}}$. We have

$$
\begin{gathered}
\left(\tilde{\omega}^{1}\right)_{1} \mathbf{v}^{14,1}=\left(\frac{1}{9}+\frac{2}{3}\right) \mathbf{v}^{14,1}, \quad\left(\tilde{\omega}^{2}\right)_{1} \mathbf{v}^{14,1}=0, \\
J_{2} \mathbf{v}^{14,1}=\frac{238}{81} \sqrt{-3} \mathbf{v}^{14,1}, \quad K_{2} \mathbf{v}^{14,1}=0, \\
P_{1} \mathbf{v}^{14,1}=-\frac{4}{3} \mathbf{v}^{14,2}, \quad\left(J_{1} P\right)_{2} \mathbf{v}^{14,1}=-\frac{104}{9} \sqrt{-3} \mathbf{v}^{14,2}, \quad\left(K_{1} P\right)_{2} \mathbf{v}^{14,1}=0, \\
\left(\tilde{\omega}^{1}\right)_{1} \mathbf{v}^{14,2}=\left(\frac{2}{45}+\frac{1}{3}\right) \mathbf{v}^{14,2}, \quad\left(\tilde{\omega}^{2}\right)_{1} \mathbf{v}^{14,2}=\frac{2}{5} \mathbf{v}^{14,2}, \\
J_{2} \mathbf{v}^{14,2}=\frac{22}{81} \sqrt{-3} \mathbf{v}^{14,2}, \quad K_{2} \mathbf{v}^{14,2}=0, \\
P_{1} \mathbf{v}^{14,2}=2 \mathbf{v}^{14,1}, \quad\left(J_{1} P\right)_{2} \mathbf{v}^{14,2}=\frac{52}{3} \sqrt{-3} \mathbf{v}^{14,1}, \quad\left(K_{1} P\right)_{2} \mathbf{v}^{14,2}=\frac{20}{3} \sqrt{-3} \mathbf{v}^{14,2} .
\end{gathered}
$$


The simple module $V_{L}^{\mathbf{0}}\left(\tau^{2}\right)(2)$. By Equation (3-24), we have the isomorphism $V_{L}^{0}\left(\tau^{2}\right)(2) \cong\left(M_{T}\left(\tau^{2}\right)(2) \otimes M_{t}^{0}\right) \oplus\left(W_{T}\left(\tau^{2}\right)(2) \otimes W_{t}^{0}\right)$ as $M^{0}$-modules. The top level of $V_{L}^{0}\left(\tau^{2}\right)(2)$ is $\mathbb{C} \mathbf{v}^{15}$, where $\mathbf{v}^{15}=h_{2}^{\prime}(-1 / 3) \otimes v \in W_{T}\left(\tau^{2}\right)(2) \otimes W_{t}^{0}$ and $0 \neq v \in T_{\chi_{0}^{\prime}}$. We have

$$
\begin{gathered}
\left(\tilde{\omega}^{1}\right)_{1} \mathbf{v}^{15}=\frac{2}{45} \mathbf{v}^{15}, \quad\left(\tilde{\omega}^{2}\right)_{1} \mathbf{v}^{15}=\frac{2}{5} \mathbf{v}^{15}, \quad J_{2} \mathbf{v}^{15}=\frac{4}{81} \sqrt{-3} \mathbf{v}^{15}, \quad K_{2} \mathbf{v}^{15}=0, \\
P_{1} \mathbf{v}^{15}=0, \quad\left(J_{1} P\right)_{2} \mathbf{v}^{15}=0, \quad\left(K_{1} P\right)_{2} \mathbf{v}^{15}=-\frac{4}{3} \sqrt{-3} \mathbf{v}^{15} .
\end{gathered}
$$

The simple module $V_{L}^{j}\left(\tau^{2}\right)(0), j=1,2$. By (3-24), we have the isomorphism $V_{L}^{j}\left(\tau^{2}\right)(0) \cong\left(M_{T}\left(\tau^{2}\right)(2) \otimes M_{t}^{j}\right) \oplus\left(W_{T}\left(\tau^{2}\right)(2) \otimes W_{t}^{j}\right)$ as $M^{0}$-modules for $j=1,2$. The top level of $V_{L}^{j}\left(\tau^{2}\right)(0)$ is $\mathbb{C} \mathbf{v}^{16, j}$, where $\mathbf{v}^{16, j}=1 \otimes v \in W_{T}\left(\tau^{2}\right)(2) \otimes W_{t}^{j}$ and $0 \neq v \in T_{\chi_{j}^{\prime}}$. We have

$$
\begin{gathered}
\left(\tilde{\omega}^{1}\right)_{1} \mathbf{v}^{16, j}=\frac{2}{45} \mathbf{v}^{16, j}, \quad\left(\tilde{\omega}^{2}\right)_{1} \mathbf{v}^{16, j}=\frac{1}{15} \mathbf{v}^{16, j}, \quad J_{2} \mathbf{v}^{16, j}=\frac{4}{81} \sqrt{-3} \mathbf{v}^{16, j}, \\
K_{2} \mathbf{v}^{16, j}=(-1)^{j} \frac{2}{9} \mathbf{v}^{16, j}, \quad P_{1} \mathbf{v}^{16, j}=(-1)^{j} \frac{1}{9} \sqrt{-3} \mathbf{v}^{16, j}, \\
\left(J_{1} P\right)_{2} \mathbf{v}^{16, j}=-(-1)^{j} \frac{8}{9} \mathbf{v}^{16, j}, \quad\left(K_{1} P\right)_{2} \mathbf{v}^{16, j}=\frac{2}{9} \sqrt{-3} \mathbf{v}^{16, j} .
\end{gathered}
$$

The simple module $V_{L}^{j}\left(\tau^{2}\right)(1), j=1,2$. By (3-24), we have the isomorphism $V_{L}^{j}\left(\tau^{2}\right)(1) \cong\left(M_{T}\left(\tau^{2}\right)(0) \otimes M_{t}^{j}\right) \oplus\left(W_{T}\left(\tau^{2}\right)(0) \otimes W_{t}^{j}\right)$ as $M^{0}$-modules for $j=1,2$. The top level of $V_{L}^{j}\left(\tau^{2}\right)(1)$ is of dimension 2 with basis $\left\{\mathbf{v}^{17, j, 1}, \mathbf{v}^{17, j, 2}\right\}$, where

$$
\begin{aligned}
& \mathbf{v}^{17, j, 1}=h_{1}^{\prime}(-2 / 3) \otimes v-(-1)^{j} \sqrt{-3} h_{2}^{\prime}(-1 / 3)^{2} \otimes v \in M_{T}\left(\tau^{2}\right)(0) \otimes M_{t}^{j}, \\
& \mathbf{v}^{17, j, 2}=2 h_{1}^{\prime}(-2 / 3) \otimes v+(-1)^{j} \sqrt{-3} h_{2}^{\prime}(-1 / 3)^{2} \otimes v \in W_{T}\left(\tau^{2}\right)(0) \otimes W_{t}^{j}
\end{aligned}
$$

and $0 \neq v \in T_{\chi_{j}^{\prime}}$. We have

$$
\begin{gathered}
\left(\tilde{\omega}^{1}\right)_{1} \mathbf{v}^{17, j, 1}=\frac{1}{9} \mathbf{v}^{17, j, 1}, \quad\left(\tilde{\omega}^{2}\right)_{1} \mathbf{v}^{17, j, 1}=\frac{2}{3} \mathbf{v}^{17, j, 1}, \quad J_{2} \mathbf{v}^{17, j, 1}=-\frac{14}{81} \sqrt{-3} \mathbf{v}^{17, j, 1}, \\
K_{2} \mathbf{v}^{17, j, 1}=-(-1)^{j} \frac{52}{9} \mathbf{v}^{17, j, 1}, \quad P_{1} \mathbf{v}^{17, j, 1}=-(-1)^{j} \frac{4}{9} \sqrt{-3} \mathbf{v}^{17, j, 2} \\
\left(J_{1} P\right)_{2} \mathbf{v}^{17, j, 1}=-(-1)^{j} \frac{52}{9} \mathbf{v}^{17, j, 2}, \quad\left(K_{1} P\right)_{2} \mathbf{v}^{17, j, 1}=\frac{28}{9} \sqrt{-3} \mathbf{v}^{17, j, 2} \\
\left(\tilde{\omega}^{1}\right)_{1} \mathbf{v}^{17, j, 2}=\left(\frac{2}{45}+\frac{2}{3}\right) \mathbf{v}^{17, j, 2}, \quad\left(\tilde{\omega}^{2}\right)_{1} \mathbf{v}^{17, j, 2}=\frac{1}{15} \mathbf{v}^{17, j, 2} \\
J_{2} \mathbf{v}^{17, j, 2}=-\frac{176}{81} \sqrt{-3} \mathbf{v}^{17, j, 2}, \quad K_{2} \mathbf{v}^{17, j, 2}=(-1)^{j} \frac{2}{9} \mathbf{v}^{17, j, 2} \\
P_{1} \mathbf{v}^{17, j, 2}=-(-1)^{j} \frac{8}{9} \sqrt{-3} \mathbf{v}^{17, j, 1}+(-1)^{j} \frac{5}{9} \sqrt{-3} \mathbf{v}^{17, j, 2} \\
\left(J_{1} P\right)_{2} \mathbf{v}^{17, j, 2}=-(-1)^{j} \frac{104}{9} \mathbf{v}^{17, j, 1}+(-1)^{j} \frac{200}{9} \mathbf{v}^{17, j, 2} \\
\left(K_{1} P\right)_{2} \mathbf{v}^{17, j, 2}=\frac{56}{9} \sqrt{-3} \mathbf{v}^{17, j, 1}+\frac{10}{9} \sqrt{-3} \mathbf{v}^{17, j, 2}
\end{gathered}
$$

The simple module $V_{L}^{j}\left(\tau^{2}\right)(2), j=1,2$. By (3-24), we have the isomorphism $V_{L}^{j}\left(\tau^{2}\right)(2) \cong\left(M_{T}\left(\tau^{2}\right)(1) \otimes M_{t}^{j}\right) \oplus\left(W_{T}\left(\tau^{2}\right)(1) \otimes W_{t}^{j}\right)$ as $M^{0}$-modules for $j=1,2$. 
The top level of $V_{L}^{j}\left(\tau^{2}\right)(2)$ is $\mathbb{C} \mathbf{v}^{18, j}$, where $\mathbf{v}^{18, j}=h_{2}^{\prime}(-1 / 3) \otimes v \in W_{T}\left(\tau^{2}\right)(1) \otimes W_{t}^{j}$ and $0 \neq v \in T_{\chi_{j}^{\prime}}$. We have

$$
\begin{gathered}
\left(\tilde{\omega}^{1}\right)_{1} \mathbf{v}^{18, j}=\left(\frac{2}{45}+\frac{1}{3}\right) \mathbf{v}^{18, j}, \quad\left(\tilde{\omega}^{2}\right)_{1} \mathbf{v}^{18, j}=\frac{1}{15} \mathbf{v}^{18, j}, \quad J_{2} \mathbf{v}^{18, j}=\frac{22}{81} \sqrt{-3} \mathbf{v}^{18, j}, \\
K_{2} \mathbf{v}^{18, j}=(-1)^{j} \frac{2}{9} \mathbf{v}^{18, j}, \quad P_{1} \mathbf{v}^{18, j}=-(-1)^{j} \frac{5}{9} \sqrt{-3} \mathbf{v}^{18, j} \\
\left(J_{1} P\right)_{2} \mathbf{v}^{18, j}=-(-1)^{j} \frac{8}{9} \mathbf{v}^{18, j}, \quad\left(K_{1} P\right)_{2} \mathbf{v}^{18, j}=-\frac{10}{9} \sqrt{-3} \mathbf{v}^{18, j} .
\end{gathered}
$$

Symmetries by $\sigma$. We now consider the automorphisms $\sigma$ and $\theta$ of $V_{L}$ that are lifts of the isometries $\sigma$ and $\theta$ of the lattice $L$ defined by (3-1). Clearly, $\sigma \tau \sigma=\tau^{2}$, $\sigma \theta=\theta \sigma$, and $\tau \theta=\theta \tau$. Thus $\sigma$ and $\theta$ induce automorphisms of $V_{L}^{\tau}$ of order 2. We have $\sigma J=-J, \sigma K=-K, \sigma P=P, \theta J=J, \theta K=-K$, and $\theta P=-P$. Hence $\sigma$ and $\theta$ induce the same automorphism of $M_{t}^{0}$ and $\theta$ is the identity on $M(0)$. Note also that $\sigma\left(J_{1} P\right)=-J_{1} P$ and $\sigma\left(K_{1} P\right)=-K_{1} P$.

From the action of $\sigma$ on the top level of the 30 known simple $V_{L}^{\tau}$-modules or the action of $J_{2}, K_{2},\left(J_{1} P\right)_{2}$, and $\left(K_{1} P\right)_{2}$, we know how $\sigma$ permutes those simple $V_{L}^{\tau}$-modules. In fact, $\sigma$ transforms $V_{L^{(c, 0)}}$ into an equivalent simple $V_{L}^{\tau}$-module and interchanges the remaining simple $V_{L}^{\tau}$-modules as follows.

$$
\begin{aligned}
& V_{L}(1) \leftrightarrow V_{L}(2), \\
& V_{L^{(0,1)}}(\varepsilon) \leftrightarrow V_{L^{(0,2)}}(2 \varepsilon), \\
& \varepsilon=0,1,2 \text {, } \\
& V_{L^{(c, 1)}} \leftrightarrow V_{L^{(c, 2)}}, \\
& V_{L}^{j}(\tau)(\varepsilon) \leftrightarrow V_{L}^{j}\left(\tau^{2}\right)(\varepsilon), \\
& j, \varepsilon=0,1,2 \text {. }
\end{aligned}
$$

Note that $\sigma h_{i}=\xi^{3-i} h_{i}^{\prime}, i=1,2$. The top level of $V_{L}^{j}\left(\tau^{2}\right)(\varepsilon)$ can be obtained by replacing $h_{i}(i / 3+n)$ with $h_{i}^{\prime}(i / 3+n)$ in the top level of $V_{L}^{j}(\tau)(\varepsilon)$ for $j, \varepsilon=$ $0,1,2$. The corresponding action of $\sigma$ on the simple $M(0)$-modules was discussed in [Dong et al. 2004, Section 4.4].

\section{Classification of simple modules}

We keep the notation in the preceding section. Thus $V_{L}^{\tau}=M^{0} \oplus W^{0}$ with $M^{0}=$ $M(0) \otimes M_{t}^{0}$ and $W^{0}=W(0) \otimes W_{t}^{0}$. In this section we show that any simple $V_{L^{\tau}}^{\tau}$ module is equivalent to one of the 30 simple $V_{L}^{\tau}$-modules listed in Lemma 3.2. The result will be established by considering the Zhu algebra $A\left(V_{L}^{\tau}\right)$ of $V_{L}^{\tau}$.

First, we review some notation and basic formulas for the Zhu algebra $A(V)$ of a vertex operator algebra $(V, Y, \mathbf{1}, \omega)$. Define two binary operations

$$
u * v=\sum_{i=0}^{\infty}\left(\begin{array}{c}
\mathrm{wt} u \\
i
\end{array}\right) u_{i-1} v, \quad u \circ v=\sum_{i=0}^{\infty}\left(\begin{array}{c}
\mathrm{wt} u \\
i
\end{array}\right) u_{i-2} v
$$

for $u, v \in V$ with $u$ being homogeneous and extend $*$ and $\circ$ for arbitrary $u \in V$ by linearity. Let $O(V)$ be the subspace of $V$ spanned by all $u \circ v$ for $u, v \in V$. Set $A(V)=V / O(V)$. By [Zhu 1996, Theorem 2.1.1], $O(V)$ is a two-sided ideal with 
respect to the operation $*$. Thus $*$ induces an operation in $A(V)$. Denote by [ $v]$ the image of $v \in V$ in $A(V)$. Then $[u] *[v]=[u * v]$ and $A(V)$ is an associative algebra by this operation. Moreover, [1] is the identity and $[\omega]$ is in the center of $A(V)$. For $u, v \in V$, we write $u \sim v$ if $[u]=[v]$. For $\varphi, \psi \in$ End $V$, we write $\varphi \sim \psi$ if $\varphi v \sim \psi v$ for all $v \in V$. We need some basic formulas from [Zhu 1996].

$$
\begin{gathered}
v * u \sim \sum_{i=0}^{\infty}\left(\begin{array}{c}
\mathrm{wt}(u)-1 \\
i
\end{array}\right) u_{i-1} v, \\
\sum_{i=0}^{\infty}\left(\begin{array}{c}
\mathrm{wt}(u)+m \\
i
\end{array}\right) u_{i-n-2} v \in O(V), \quad n \geq m \geq 0 .
\end{gathered}
$$

Moreover (see [Wang 1993]),

$$
\begin{gathered}
L(-n) \sim(-1)^{n}\{(n-1)(L(-2)+L(-1))+L(0)\}, \quad n \geq 1, \\
{[\omega] *[u]=[(L(-2)+L(-1)) u],}
\end{gathered}
$$

where $L(n)=\omega_{n+1}$. From (5-4) and (5-5) we have

$$
[L(-n) u]=(-1)^{n}(n-1)[\omega] *[u]+(-1)^{n}[L(0) u], \quad n \geq 1 .
$$

If $u \in V$ is of weight 2 , then $u(-n-3)+2 u(-n-2)+u(-n-1) \sim 0$ by (5-3), where $u(n)=u_{n+1}$. Hence

$$
u(-n) \sim(-1)^{n}((n-1) u(-2)+(n-2) u(-1))
$$

for $n \geq 1$. Then it follows from (5-1) and (5-2) that

$$
u(-n) w \sim(-1)^{n}(-u * w+n w * u+u(0) w)
$$

for $n \geq 1, w \in V$. Likewise, if $u$ is of weight 3 and $u(n)=u_{n+2}$, then

$$
\begin{aligned}
& u(-n) \sim(-1)^{n+1} \\
& \quad \cdot\left(\frac{1}{2}(n-1)(n-2) u(-3)+(n-1)(n-3) u(-2)+\frac{1}{2}(n-2)(n-3) u(-1)\right),
\end{aligned}
$$

(5-10) $u(-n) w \sim(-1)^{n+1}\left(n u(-1) w+(n-1) u(0) w-(n-1) u * w+\frac{1}{2} n(n-1) w * u\right)$, for $n \geq 1, w \in V$.

For a homogeneous vector $u \in V, o(u)=u_{\mathrm{wt}(u)-1}$ is the weight zero component operator of $Y(u, z)$. Extend $o(u)$ for arbitrary $u \in V$ by linearity. Note that we call a module in the sense of [Zhu 1996] an $\mathbb{N}$-graded weak module here. If $M=$ $\oplus_{n=0}^{\infty} M_{(n)}$ is an $\mathbb{N}$-graded weak $V$-module with $M_{(0)} \neq 0$, then $o(u)$ acts on its top level $M_{(0)}$. Zhu's theory [1996] says: (1) $o(u) o(v)=o(u * v)$ as operators on the top level $M_{(0)}$ and $o(u)$ acts as 0 on $M_{(0)}$ if $u \in O(V)$. Thus $M_{(0)}$ is an $A(V)$ module, where $[u]$ acts on $M_{(0)}$ as $o(u)$. (2) The map $M \mapsto M_{(0)}$ is a bijection 
between the set of isomorphism classes of simple $\mathbb{N}$-graded weak $V$-modules and the set of isomorphism classes of simple $A(V)$-modules.

We return to $V_{L}^{\tau}$. As in Section 3, we write $L^{i}(n)=\left(\tilde{\omega}^{i}\right)_{n+1}, i=1,2, J(n)=$ $J_{n+2}$, and $K(n)=K_{n+2}$. The Zhu algebras $A(M(0))$ and $A\left(M_{t}^{0}\right)$ were determined in [Dong et al. 2004] and [Kitazume et al. 2000b], respectively. Since $O\left(M^{0}\right) \subset O\left(V_{L}^{\tau}\right)$, the image of $M(0)$ (resp. $\left.M_{t}^{0}\right)$ in $A\left(V_{L}^{\tau}\right)$ is a homomorphic image of $A(M(0))$ (resp. $A\left(M_{t}^{0}\right)$ ). It is generated by $\left[\tilde{\omega}^{1}\right],[J]\left(\operatorname{resp} .\left[\tilde{\omega}^{2}\right],[K]\right)$.

By a direct calculation, we have

$$
\begin{aligned}
P_{1} P & =-16 \tilde{\omega}^{1}-6 \tilde{\omega}^{2} \\
P_{0} P & =-8\left(\tilde{\omega}^{1}\right)_{-2} \mathbf{1}-3\left(\tilde{\omega}^{2}\right)_{-2} \mathbf{1}, \\
P_{-1} P= & \frac{5}{273} J_{1} K_{1} P-\frac{12}{7}\left(\tilde{\omega}^{1}\right)_{-3} \mathbf{1}-\frac{18}{13}\left(\tilde{\omega}^{2}\right)_{-3} \mathbf{1} \\
& \quad-\frac{36}{7}\left(\tilde{\omega}^{1}\right)_{-1}\left(\tilde{\omega}^{1}\right)_{-1} \mathbf{1}-\frac{9}{13}\left(\tilde{\omega}^{2}\right)_{-1}\left(\tilde{\omega}^{2}\right)_{-1} \mathbf{1}-16\left(\tilde{\omega}^{1}\right)_{-1}\left(\tilde{\omega}^{2}\right)_{-1} \mathbf{1} \\
11) \quad & \quad-\frac{9}{13}\left(\tilde{\omega}^{2}\right)_{-2}\left(\tilde{\omega}^{2}\right)_{-1} \mathbf{1}-8\left(\tilde{\omega}^{1}\right)_{-2}\left(\tilde{\omega}^{2}\right)_{-1} \mathbf{1}-8\left(\tilde{\omega}^{1}\right)_{-1}\left(\tilde{\omega}^{2}\right)_{-2} \mathbf{1} .
\end{aligned}
$$

Moreover, $J_{2} P=K_{2} P=0$. Then, using formulas (5-4)-(5-10), we obtain

$$
\begin{aligned}
{[P] *[P]=\frac{5}{273}\left[J_{1} K_{1} P\right]-\frac{36}{7}\left[\tilde{\omega}^{1}\right] *\left[\tilde{\omega}^{1}\right]-\frac{9}{13}\left[\tilde{\omega}^{2}\right] *\left[\tilde{\omega}^{2}\right] } \\
\\
-16\left[\tilde{\omega}^{1}\right] *\left[\tilde{\omega}^{2}\right]+\frac{4}{7}\left[\tilde{\omega}^{1}\right]+\frac{6}{13}\left[\tilde{\omega}^{2}\right],
\end{aligned}
$$

$$
\begin{aligned}
{[P \circ P] } & =\frac{1}{84}[J] *\left[K_{1} P\right]-\frac{1}{84}\left[K_{1} P\right] *[J]+\frac{1}{156}[K] *\left[J_{1} P\right]-\frac{1}{156}\left[J_{1} P\right] *[K] \\
& =0 .
\end{aligned}
$$

It turns out that $A\left(V_{L}^{\tau}\right)$ is generated by $\left[\tilde{\omega}^{1}\right],\left[\tilde{\omega}^{2}\right],[J],[K]$, and $[P]$ (Corollary 5.11). However, we first prove the following intermediate assertion.

Proposition 5.1. The Zhu algebra $A\left(V_{L}^{\tau}\right)$ is generated by $\left[\tilde{\omega}^{1}\right],\left[\tilde{\omega}^{2}\right],[J],[K],[P]$, $\left[J_{1} P\right]$, and $\left[K_{1} P\right]$.

Proof. Recall that $L^{i}(n) P=0$ for $i=1,2, n \geq 1, L^{1}(0) P=\frac{8}{5} P, L^{2}(0) P=\frac{2}{5} P$, and $J(n) P=K(n) P=0$ for $n \geq 0$. Thus from the commutation relations (3-9)-(3-11) and (3-13)-(3-15) we see that $W^{0}$ is spanned by the vectors of the form

$$
\begin{aligned}
& L^{1}\left(-j_{1}\right) \cdots L^{1}\left(-j_{r}\right) L^{2}\left(-k_{1}\right) \cdots L^{2}\left(-k_{s}\right) \\
& \cdot J\left(-m_{1}\right) \cdots J\left(-m_{p}\right) K\left(-n_{1}\right) \cdots K\left(-n_{q}\right) P
\end{aligned}
$$

with $j_{1} \geq \cdots \geq j_{r} \geq 1, k_{1} \geq \cdots \geq k_{s} \geq 1, m_{1} \geq \cdots \geq m_{p} \geq 1, n_{1} \geq \cdots \geq n_{q} \geq 1$. Let $v$ be a vector of this form. Its weight is

$$
j_{1}+\cdots+j_{r}+k_{1}+\cdots+k_{s}+m_{1}+\cdots+m_{p}+n_{1}+\cdots+n_{q}+2 .
$$


Since $V_{L}^{\tau}=M^{0} \oplus W^{0}$ and since the image of $M^{0}$ in $A\left(V_{L}^{\tau}\right)$ is generated by $\left[\tilde{\omega}^{1}\right]$, $\left[\tilde{\omega}^{2}\right],[J]$, and $[K]$, it suffices to show that the image $[v]$ of $v$ in $A\left(V_{L}^{\tau}\right)$ is contained in the subalgebra generated by $\left[\tilde{\omega}^{1}\right],\left[\tilde{\omega}^{2}\right],[J],[K],[P],\left[J_{1} P\right]$, and $\left[K_{1} P\right]$. We proceed by induction on the weight of $v$. By formula (5-8) with $u=\tilde{\omega}^{i}, i=1,2$ and the induction on the weight, we may assume that $r=s=0$, that is,

$$
v=J\left(-m_{1}\right) \cdots J\left(-m_{p}\right) K\left(-n_{1}\right) \cdots K\left(-n_{q}\right) P .
$$

Moreover, by formula (5-10) with $u=J$, we may assume that $m_{1}=\cdots=m_{p}=1$. Since $J(m)$ and $K(n)$ commute, we may also assume that $n_{1}=\cdots=n_{q}=1$ by a similar argument. Then $v=J(-1)^{p} K(-1)^{q} P$.

Next, we reduce $v$ to the case $p \leq 1$. For this purpose, we use a singular vector

$$
5 J(-1)^{2} P+2496 L^{1}(-2) P-195 L^{1}(-1)^{2} P=0 .
$$

in $W(0)$. Suppose $p \geq 2$. Then, since $K(-1)$ commutes with $J(m)$ and $L^{1}(n)$, (5-15) implies that $v=J(-1)^{p} K(-1)^{q} P$ is a linear combination of

$$
J(-1)^{p-2} L^{1}(-2) K(-1)^{q} P \quad \text { and } \quad J(-1)^{p-2} L^{1}(-1)^{2} K(-1)^{q} P .
$$

By (3-10), these two vectors can be written in the form $L^{1}(-2) H K(-1)^{q} P$ and $L^{1}(-1)^{2} H^{\prime} K(-1)^{q} P$, where $H$ (resp. $\left.H^{\prime}\right)$ is a polynomial in $J(-1)$ and $J(-3)$ (resp. $J(-1), J(-2)$, and $J(-3)$ ). Then by (5-8) with $u=\tilde{\omega}^{1}$ and the induction on the weight, the assertion holds for $v$. Hence we may assume that $p \leq 1$.

There is a singular vector

$$
K(-1)^{2} P-210 L^{2}(-2) P=0
$$

in $W_{t}^{0}$. Thus, by a similar argument as above, we may assume that $q \leq 1$. Finally, it follows from $(5-12)$ that $[J(-1) K(-1) P]$ can be written by $\left[\tilde{\omega}^{1}\right],\left[\tilde{\omega}^{2}\right]$, and $[P]$ in $A\left(V_{L}^{\tau}\right)$. The proof is complete.

We will classify the simple $V_{L}^{\tau}$-modules using our knowledge of simple modules for $M(0)$ and $M_{t}^{0}$ together with fusion rules (3-25) and (3-7). Set

$$
\begin{aligned}
& M_{1}=\left\{M(\varepsilon), M_{k}^{c}, M_{T}\left(\tau^{i}\right)(\varepsilon) \mid i=1,2, \varepsilon=0,1,2\right\}, \\
& \mathcal{W}_{1}=\left\{W(\varepsilon), W_{k}^{c}, W_{T}\left(\tau^{i}\right)(\varepsilon) \mid i=1,2, \varepsilon=0,1,2\right\}, \\
& \mathcal{M}_{2}=\left\{M_{t}^{j} \mid j=0,1,2\right\}, \quad W_{2}=\left\{W_{t}^{j} \mid j=0,1,2\right\} .
\end{aligned}
$$

Then $M_{1} \cup W_{1}$ (resp. $M_{2} \cup W_{2}$ ) is a complete set of representatives of isomorphism classes of simple $M(0)$-modules (resp. simple $M_{t}^{0}$-modules). A main point is that the fusion rules of the following form hold.

$$
\begin{aligned}
W(0) \times M^{1} & =W^{1}, & & W(0) \times W^{1}=M^{1}+W^{1}, \\
W_{t}^{0} \times M^{2} & =W^{2}, & & W_{t}^{0} \times W^{2}=M^{2}+W^{2},
\end{aligned}
$$


where $M^{i} \in \mathcal{M}_{i}, i=1,2$, and $W^{i} \in \mathcal{W}_{i}$ is determined by $M^{i}$ through the fusion rule $W(0) \times M^{1}=W^{1}$ or $W_{t}^{0} \times M^{2}=W^{2}$.

Recall that $M^{0}$ is rational, $C_{2}$-cofinite, and of CFT type. Thus every $\mathbb{N}$-graded weak $M^{0}$-module is a direct sum of simple $M^{0}$-modules. As a result, every $\mathbb{N}$ graded weak $V_{L}^{\tau}$-module is decomposed into a direct sum of simple $M^{0}$-modules, and in particular $L(0)=\omega_{1}$ acts semisimply on it. Each weight subspace, that is, each eigenspace for $L(0)$ is not necessarily a finite dimensional space. However, any simple weak $V_{L}^{\tau}$-module is a simple ordinary $V_{L}^{\tau}$-module by [Abe et al. 2004, Corollary 5.8], since $V_{L}^{\tau}$ is $C_{2}$-cofinite and of CFT type.

We note that

$$
W^{0} \cdot W^{0}=V_{L}^{\tau} .
$$

Indeed, $W^{0} \cdot W^{0}=\operatorname{span}\left\{a_{n} b \mid a, b \in W^{0}, n \in \mathbb{Z}\right\}$ is an $M^{0}$-submodule of $V_{L}^{\tau}$ by (2-6). Since $P, J_{1} K_{1} P \in W^{0}$ and $\tilde{\omega}^{1}, \tilde{\omega}^{2} \in M^{0},(5-11)$ implies that $W^{0} \cdot W^{0}=M^{0} \oplus W^{0}$.

Each simple $M^{0}$-module is isomorphic to a tensor product $A \otimes B$ of a simple $M(0)$-module $A$ and a simple $M_{t}^{0}$-module $B$. We show that only restricted simple $M^{0}$-modules can appear in $\mathbb{N}$-graded weak $V_{L}^{\tau}$-modules.

Lemma 5.2. Let $U$ be an $\mathbb{N}$-graded weak $V_{L}^{\tau}$-module. Then any simple $M^{0}$ submodule of $U$ is isomorphic to $M^{1} \otimes M^{2}$ or $W^{1} \otimes W^{2}$ for some $M^{i} \in \mathcal{M}_{i}$ and $W^{i} \in W_{i}, i=1,2$.

Proof. Suppose $U$ contains a simple $M^{0}$-submodule $S^{0} \cong M^{1} \otimes W^{2}$ with $M^{1} \in M_{1}$ and $W^{2} \in W_{2}$. Let $S=V_{L}^{\tau} \cdot S^{0}=\operatorname{span}\left\{a_{n} w \mid a \in V_{L}^{\tau}, w \in S^{0}, n \in \mathbb{Z}\right\}$. Then (2-6) implies that $S$ is the $\mathbb{N}$-graded weak $V_{L}^{\tau}$-submodule of $U$ generated by $S^{0}$. By the construction of $S$, the difference of any two eigenvalues of $L(0)$ in $S$ is an integer. In fact, $S$ is an ordinary $V_{L}^{\tau}$-module by Remark 2.16 .

If $v$ is a nonzero vector in $V_{L}^{\tau}$, then $v_{n} S^{0} \neq 0$ for some $n \in \mathbb{Z}$. Indeed, Lemma 2.6 implies that the set $\left\{v \in V_{L}^{\tau} \mid v_{n} S^{0}=0\right.$ for all $\left.n \in \mathbb{Z}\right\}$ is an ideal of $V_{L}^{\tau}$. It is in fact 0 , since $V_{L}^{\tau}$ is a simple vertex operator algebra and $S^{0}$ is a simple $M^{0}$-module. Then by the fusion rules (5-17), a simple $M^{0}$-module isomorphic to $W^{1} \otimes M^{2}$ or $W^{1} \otimes W^{2}$ must appear in $S$. However, the difference of the minimal eigenvalues of $L(0)$ in $M^{1} \otimes W^{2}$ and $W^{1} \otimes M^{2}$, or in $M^{1} \otimes W^{2}$ and $W^{1} \otimes W^{2}$ is not an integer. This is a contradiction. Thus $U$ does not contain a simple $M^{0}$-submodule isomorphic to $M^{1} \otimes W^{2}$. By a similar argument, we can also show that there is no simple $M^{0}$-submodule isomorphic to $W^{1} \otimes M^{2}$ in $U$. Hence the assertion holds.

Set $\mathcal{M}=\left\{M^{1} \otimes M^{2} \mid M^{i} \in \mathcal{M}_{i}, i=1,2\right\}$ and $\mathscr{W}=\left\{W^{1} \otimes W^{2} \mid W^{i} \in W_{i}, i=1,2\right\}$. Then each of $\mathcal{M}$ and $W$ consists of 30 inequivalent simple $M^{0}$-modules. The top level of every simple $M^{0}$-module is of dimension one.

Lemma 5.3. If $U$ is a simple $\mathbb{N}$-graded weak $V_{L}^{\tau}$-module whose top level is of dimension one, then $U$ is isomorphic to one of the 23 known simple $V_{L}^{\tau}$-modules 
with one dimensional top level, namely, $V_{L^{(0, j)}}(\varepsilon), j=0,1,2, \varepsilon=0,1,2, V_{L^{(c, j)}}$, $j=1,2, V_{L}^{j}(\tau)(\varepsilon), j=0,1,2, \varepsilon=0,2$, and $V_{L}^{j}\left(\tau^{2}\right)(\varepsilon), j=0,1,2, \varepsilon=0,2$.

Proof. Since $U$ is a direct sum of simple $M^{0}$-modules and since the top level, say $U_{\lambda}$ of $U$ is assumed to be of dimension one, it follows from Lemma 5.2 that $U_{\lambda}$ is isomorphic to the top level of $M^{1} \otimes M^{2}$ or the top level of $W^{1} \otimes W^{2}$ as an $A\left(M^{0}\right)$-module for some $M^{i} \in \mathcal{M}_{i}, W^{i} \in \mathcal{W}_{i}, i=1,2$. The Zhu algebra

$$
A\left(M^{0}\right) \cong A(M(0)) \times A\left(M_{t}^{0}\right)
$$

is commutative and the action of $A\left(M^{0}\right)$ on the top level of $M^{1} \otimes M^{2}$ and the top level of $W^{1} \otimes W^{2}$ are known. Indeed, we know all possible action of the elements $\left[\tilde{\omega}^{1}\right],\left[\tilde{\omega}^{2}\right],[J]$, and $[K]$ of $A\left(V_{L}^{\tau}\right)$ on $U_{\lambda}$. Let $\left[\tilde{\omega}^{1}\right],\left[\tilde{\omega}^{2}\right],[J]$, and $[K]$ act on $U_{\lambda}$ as scalars $a_{1}, a_{2}, b_{1}$, and $b_{2}$, respectively. There are 60 possible such quadruplets $\left(a_{1}, a_{2}, b_{1}, b_{2}\right)$.

Let $[P],\left[J_{1} P\right]$, and $\left[K_{1} P\right]$ act on $U_{\lambda}$ as scalars $x_{1}, x_{2}$, and $x_{3}$, respectively. Then it follows from (5-12) that $\left[J_{1} K_{1} P\right]$ acts on $U_{\lambda}$ as a scalar

$$
\frac{273}{5} x_{1}^{2}+\frac{1404}{5} a_{1}^{2}+\frac{189}{5} a_{2}^{2}+\frac{4368}{5} a_{1} a_{2}-\frac{156}{5} a_{1}-\frac{126}{5} a_{2} .
$$

From computer calculations, whose results are presented in an online supplement to this paper, ${ }^{1}$ and from formulas (5-4)-(5-10), we conclude that the vanishing of $\left[P \circ\left(J_{1} P\right)\right]$ and $\left[P \circ\left(K_{1} P\right)\right]$ imply, respectively,

$$
15 b_{2} x_{1}+5 a_{2} x_{3}-2 x_{3}=0, \quad\left(15 a_{2}-1\right) x_{2}=0 .
$$

Using (5-19), we can calculate

$$
\left[\left(J_{1} P\right) *\left(J_{1} P\right)\right], \quad\left[\left(K_{1} P\right) *\left(K_{1} P\right)\right], \quad\left[\left(J_{1} P\right) *\left(K_{1} P\right)\right]
$$

in a similar way and verify that the following equations hold.

$$
\begin{aligned}
x_{2}^{2}=\left(\frac{229164}{575} a_{1}\right. & \left.-\frac{37856}{425} a_{2}+\frac{1669382}{48875}\right) x_{1}^{2}-\frac{56}{85} b_{2} x_{2}-\frac{4056}{115} b_{1} x_{3}+\frac{348994464}{107525} a_{1}^{3} \\
& +\frac{137149584}{9775} a_{1}^{2} a_{2}-\frac{1030224}{1375} a_{1}^{2}+\frac{7064876}{9775} a_{1} a_{2}^{2}-\frac{40788488}{48875} a_{1} a_{2} \\
& +\frac{16160456}{537625} a_{1}-\frac{419184}{9775} a_{2}^{3}-\frac{200994}{48875} a_{2}^{2}+\frac{1065516}{48875} a_{2}-\frac{3042}{187} b_{1}^{2},
\end{aligned}
$$

$$
\begin{aligned}
& x_{3}^{2}=\left(-\frac{37044}{575} a_{1}-\frac{5684}{85} a_{2}+\frac{741713}{97750}\right) x_{1}^{2} \\
& +\frac{28}{221} b_{2} x_{2}+\frac{216}{115} b_{1} x_{3}-\frac{54559344}{107525} a_{1}^{3}-\frac{28217448}{9775} a_{1}^{2} a_{2}+\frac{254982}{1375} a_{1}^{2}-\frac{25042724}{9775} a_{1} a_{2}^{2} \\
& +\frac{26308184}{48875} a_{1} a_{2}-\frac{8127098}{537625} a_{1}-\frac{4775148}{25415} a_{2}^{3}+\frac{188338017}{1270750} a_{2}^{2}-\frac{9722139}{635375} a_{2}-\frac{180}{187} b_{1}^{2},
\end{aligned}
$$

$$
x_{2} x_{3}=\left(-\frac{864}{5} a_{1}^{2}+\frac{1248}{25} a_{1} a_{2}+\frac{1152}{5} a_{2}^{2}+\frac{5904}{125} a_{1}+\frac{184176}{125} a_{2}-\frac{62112}{625}\right) x_{1}-36 b_{1} b_{2} .
$$

\footnotetext{
${ }^{1}$ The authors can supply these expressions in machine readable form upon request.
} 
We have obtained a system of equations (5-20)-(5-23) for $x_{1}, x_{2}, x_{3}$. We can solve this system of equations with respect to the 60 possible quadruplets $\left(a_{1}, a_{2}, b_{1}, b_{2}\right)$. Actually, there is no solution for 37 quadruplets of $\left(a_{1}, a_{2}, b_{1}, b_{2}\right)$. For each of the remaining 23 quadruplets $\left(a_{1}, a_{2}, b_{1}, b_{2}\right)$, the system of equations possesses a unique solution $\left(x_{1}, x_{2}, x_{3}\right)$. Furthermore, the 23 sets $\left(a_{1}, a_{2}, b_{1}, b_{2}\right.$, $\left.x_{1}, x_{2}, x_{3}\right)$ of values determined in this way coincide with the action of $\left[\tilde{\omega}^{1}\right],\left[\tilde{\omega}^{2}\right]$, $[J],[K],[P],\left[J_{1} P\right]$, and $\left[K_{1} P\right]$ on the top level of the 23 known simple $V_{L^{-}}$ modules with one dimensional top level described in Section 4. Since $A\left(V_{L}^{\tau}\right)$ is generated by these seven elements, this implies that $U_{\lambda}$ is isomorphic to the top level of one of the 23 simple $V_{L}^{\tau}$-modules listed in the assertion as an $A\left(V_{L}^{\tau}\right)$ module. Thus the lemma holds by Zhu's theorem.

Remark 5.4. We also obtain some equations for $x_{1} x_{2}$ and $x_{1} x_{3}$ from $\left[P *\left(J_{1} P\right)\right]$ and $\left[P *\left(K_{1} P\right)\right]$. However, they are not sufficient to determine $x_{1}, x_{2}$, and $x_{3}$.

Lemma 5.5. Every $\mathbb{N}$-graded weak $V_{L}^{\tau}$-module contains a simple $M^{0}$-submodule isomorphic to a member of $M$.

Proof. Suppose false and let $U$ be an $\mathbb{N}$-graded weak $V_{L}^{\tau}$-module which contains no simple $M^{0}$-submodule isomorphic to a member of $M$. Then by Lemma 5.2, there is a simple $M^{0}$-submodule $W$ in $U$ such that $W \cong W^{1} \otimes W^{2}$ for some $W^{i} \in W_{i}$, $i=1,2$. The top level of $W$, say $W_{\lambda}$ for some $\lambda \in \mathbb{Q}$, is a one dimensional space. Take $0 \neq w \in W_{\lambda}$ and let $S=V_{L}^{\tau} \cdot w=\operatorname{span}\left\{a_{n} w \mid a \in V_{L}^{\tau}, n \in \mathbb{Z}\right\}$, which is an ordinary $V_{L}^{\tau}$-module by (2-6) and Remark 2.16. Since $V_{L}^{\tau}=M^{0} \oplus W^{0}$, it follows from our assumption and the fusion rules (5-17) that $S$ is isomorphic to a direct sum of finite number of copies of $W$ as an $M^{0}$-module. Thus $\left[\tilde{\omega}^{1}\right],\left[\tilde{\omega}^{2}\right],[J]$, and $[K]$ act on the top level $S_{\lambda}$ of $S$ as scalars, say $a_{1}, a_{2}, b_{1}$, and $b_{2}$, respectively. Then by a similar calculation as in the proof of Lemma 5.3, we see that $\left[P \circ\left(K_{1} P\right)\right]=0$ implies

$$
\left(15 a_{2}-1\right) o\left(J_{1} P\right)=0
$$

as an operator on the top level $S_{\lambda}$. Recall that $[u] \in A\left(V_{L}^{\tau}\right)$ acts on $S_{\lambda}$ as $o(u)=$ $u_{\mathrm{wt}(u)-1}$ for a homogeneous vector $u$ of $V_{L}^{\tau}$. Furthermore, we can calculate that

$$
\begin{aligned}
o\left(J_{1} P\right) o(P)-o(P) o\left(J_{1} P\right) & =0 \\
o\left(K_{1} P\right) o(P)-o(P) o\left(K_{1} P\right) & =\frac{2}{13}\left(15 a_{2}-1\right) o\left(J_{1} P\right) \\
o\left(J_{1} P\right) o\left(K_{1} P\right)-o\left(K_{1} P\right) o\left(J_{1} P\right) & =\frac{96}{125}\left(15 a_{2}-1\right)\left(65 a_{1}+100 a_{2}+441\right) o(P)
\end{aligned}
$$

as operators on $S_{\lambda}$.

By (5-24), $15 a_{2}-1=0$ or $o\left(J_{1} P\right)=0$ and so $o(P), o\left(J_{1} P\right)$, and $o\left(K_{1} P\right)$ commute each other. Thus the action of $A\left(V_{L}^{\tau}\right)$ on $S_{\lambda}$ is commutative. Hence we can choose a one dimensional $A\left(V_{L}^{\tau}\right)$-submodule $T$ of $S_{\lambda}$. Zhu's theory tells us that 
there is a simple $\mathbb{N}$-graded weak $V_{L}^{\tau}$-module $R$ whose top level $R_{\lambda}$ is isomorphic to $T$ as an $A\left(V_{L}^{\tau}\right)$-module. Since $\operatorname{dim} R_{\lambda}=1, R$ is isomorphic to one of the 23 simple $V_{L}^{\tau}$-modules listed in Lemma 5.3. In particular, $R$ contains a simple $M^{0}$ submodule $M$ isomorphic to a member of $M$. Now, consider the $V_{L}^{\tau}$-submodule $V_{L}^{\tau} \cdot T$ of $S$ generated by $T$. By Lemma 2.10, there is a surjective homomorphism of $V_{L}^{\tau}$-modules from $V_{L}^{\tau} \cdot T$ onto $R$. Then $V_{L}^{\tau} \cdot T$ must contain a simple $M^{0}$-submodule isomorphic to $M$. This contradicts our assumption. The proof is complete.

Lemma 5.6. Let $U$ be an $\mathbb{N}$-graded weak $V_{L}^{\tau}$-module and $M$ be a simple $M^{0}$ submodule of $U$ such that $M \cong M^{1} \otimes M^{2}$ as $M^{0}$-modules for some $M^{i} \in M_{i}, i=1,2$. Then $V_{L}^{\tau} \cdot M=\operatorname{span}\left\{a_{n} u \mid a \in V_{L}^{\tau}, u \in M, n \in \mathbb{Z}\right\}$ is a simple $V_{L}^{\tau}$-module. Moreover, $V_{L}^{\tau} \cdot M=M \oplus W$, where $W$ is a simple $M^{0}$-module isomorphic to $W^{1} \otimes W^{2}$ and $W^{i}, i=1,2$ are determined from $M^{i}$ by the fusion rules $W(0) \times M^{1}=W^{1}$ and $W_{t}^{0} \times M^{2}=W^{2}$ of (5-17).

Proof. By Remark 2.16, $V_{L}^{\tau} \cdot M$ is an ordinary $V_{L}^{\tau}$-module. Note that $V_{L}^{\tau} \cdot M=$ $\left(M^{0}+W^{0}\right) \cdot M=M+W^{0} \cdot M$. We see that $W^{0} \cdot M \neq 0$ by a similar argument as in the proof of Lemma 5.2. Actually, $W^{0} \cdot\left(W^{0} \cdot M\right) \supset\left(W^{0} \cdot W^{0}\right) \cdot M=V_{L}^{\tau} \cdot M$ (see Lemma 2.6 and (5-18)) implies $W^{0} \cdot M \neq 0$ also. Moreover, $W^{0} \cdot M$ is an $M^{0}$ module by (2-6). Since $M^{0}$ is rational, $W^{0} \cdot M$ is decomposed into a direct sum of simple $M^{0}$-modules, say $W^{0} \cdot M=\bigoplus_{\gamma \in \Gamma} S^{\gamma}$. Let $W=W^{1} \otimes W^{2}$, where $W^{i} \in W_{i}$, $i=1,2$ are determined by the fusion rules $W(0) \times M^{1}=W^{1}$ and $W_{t}^{0} \times M^{2}=W^{2}$. The space $I_{M^{0}}\left(\begin{array}{c}W \\ W^{0} M\end{array}\right)$ of intertwining operators of type $\left(\begin{array}{c}W \\ W^{0} M\end{array}\right)$ is of dimension one and each $S^{\gamma}$ is isomorphic to $W$.

We want to show that $|\Gamma|=1$. Suppose $\Gamma$ contains at least two elements and take $\gamma_{1}, \gamma_{2} \in \Gamma, \gamma_{1} \neq \gamma_{2}$. Let $\psi: S^{\gamma_{2}} \rightarrow S^{\gamma_{1}}$ be an isomorphism of $M^{0}$-modules and $p_{\gamma}: W^{0} \cdot M \rightarrow S^{\gamma}$ be a projection. For $a \in W^{0}$ and $u \in M$, set

$$
\mathscr{G}_{\gamma_{1}}(a, z) u=p_{\gamma_{1}} Y_{U}(a, z) u, \quad \mathscr{G}_{\gamma_{2}}(a, z) u=\psi p_{\gamma_{2}} Y_{U}(a, z) u,
$$

where $Y_{U}(a, z)$ is the vertex operator of the $\mathbb{N}$-graded weak $V_{L}^{\tau}$-module $U$. Then $\mathscr{G}_{\gamma_{i}}(\cdot, z), i=1,2$ are nonzero members in the one dimensional space $I_{M^{0}}\left(\begin{array}{c}W \\ W^{0}{ }_{M}\end{array}\right)$, so that $\mu \mathscr{G}_{\gamma_{1}}(\cdot, z)=\mathscr{G}_{\gamma_{2}}(\cdot, z)$ for some $0 \neq \mu \in \mathbb{C}$. Let $0 \neq v \in S^{\gamma_{1}}$. Then $v \in W^{0} \cdot M$ and so $v=\sum_{j}\left(a^{j}\right)_{n_{j}} u^{j}$ for some $a^{j} \in W^{0}, u^{j} \in M, n_{j} \in \mathbb{Z}$. Take the coefficients of $z^{-n_{j}-1}$ in both sides of $\mu \mathscr{G}_{\gamma_{1}}\left(a^{j}, z\right) u^{j}=\mathscr{G}_{\gamma_{2}}\left(a^{j}, z\right) u^{j}$. Then $\mu p_{\gamma_{1}}\left(\left(a^{j}\right)_{n_{j}} u^{j}\right)=\psi p_{\gamma_{2}}\left(\left(a^{j}\right)_{n_{j}} u^{j}\right)$. Summing up both sides of the equation with respect to $j$, we have $\mu p_{\gamma_{1}} v=\psi p_{\gamma_{2}} v$. However, $v \in S^{\gamma_{1}}$ implies that $p_{\gamma_{1}} v=v$ and $p_{\gamma_{2}} v=0$. This is a contradiction since $\mu \neq 0$ and $v \neq 0$. Thus $|\Gamma|=1$ and $W^{0} \cdot M \cong W$ as required.

If $V_{L}^{\tau} \cdot M$ is not a simple $V_{L}^{\tau}$-module, then there is a proper $V_{L}^{\tau}$-submodule $N$ of $V_{L}^{\tau} \cdot M$. Since $M$ and $W$ are simple $M^{0}$-modules, $N$ must be isomorphic to $M$ or $W$ as an $M^{0}$-module. Then the top level of $N$ is of dimension one. The simple 
$V_{L}^{\tau}$-modules with one dimensional top level are classified in Lemma 5.3. Each of them is a direct sum of two simple $M^{0}$-modules. However, $N$ is not of such a form. Thus $V_{L}^{\tau} \cdot M$ is a simple $V_{L}^{\tau}$-module.

Lemma 5.7. Let $U=M \oplus W$ be an $M^{0}$-module such that $M \cong M^{1} \otimes M^{2}$ and $W \cong W^{1} \otimes W^{2}$ for some $M^{i} \in \mathcal{M}_{i}$ and $W^{i} \in W_{i}, i=1,2$. Then $U$ admits at most one simple $V_{L}^{\tau}$-module structure.

Proof. Assume that $\left(U, Y_{1}\right)$ and $\left(U, Y_{2}\right)$ are simple $V_{L}^{\tau}$-modules such that $Y_{i}(a, z)=$ $Y(a, z)$ for all $a \in M^{0}, i=1,2$, where $(U, Y)$ is the given $M^{0}$-module structure. We denote the vertex operator of $V_{L}^{\tau}$ by $\tilde{Y}(v, z)$ for $v \in V_{L}^{\tau}$. Let $p_{M^{0}}: V_{L}^{\tau} \rightarrow M^{0}$ and $p_{W^{0}}: V_{L}^{\tau} \rightarrow W^{0}$ be projections and define $\mathscr{I}(\cdot, z)$ and $\mathscr{\mathscr { S }}(\cdot, z)$ by

$$
\mathscr{I}(a, z) b=p_{M^{0}} \tilde{Y}(a, z) b, \quad \mathscr{g}(a, z) b=p_{W^{0}} \tilde{Y}(a, z) b
$$

for $a, b \in W^{0}$. Then by $(5-18), \mathscr{I}(\cdot, z)$ and $\mathscr{L}(\cdot, z)$ are nonzero intertwining operators of respective types $\left(\begin{array}{c}M^{0} \\ W^{0} W^{0}\end{array}\right)$ and $\left(\begin{array}{c}W^{0} \\ W^{0} W^{0}\end{array}\right)$. By the fusion rules (5-17), the space $I_{M^{0}}\left(\begin{array}{c}M^{0} \\ W^{0} W^{0}\end{array}\right)$ of $M^{0}$-intertwining operators of type $\left(\begin{array}{c}M^{0} \\ W^{0} W^{0}\end{array}\right)$ is of dimension one. Likewise, $\operatorname{dim} I_{M^{0}}\left(\begin{array}{c}W^{0} \\ W^{0} W^{0}\end{array}\right)=1$. Note that $W^{0} \cdot M^{0} \subset W^{0}$ and that $\mathscr{I}(a, z) b+\mathscr{g}(a, z) b=\tilde{Y}(a, z) b$.

Let $p_{M}: U \rightarrow M$ and $p_{W}: U \rightarrow W$ be projections. Define $\mathscr{F}_{i}^{M}(\cdot, z)$ and $\mathscr{F}_{i}^{W}(\cdot, z), i=1,2$ by

$$
\mathscr{F}_{i}^{M}(a, z) w=p_{M} Y_{i}(a, z) w, \quad \mathscr{F}_{i}^{W}(a, z) w=p_{W} Y_{i}(a, z) w
$$

for $a \in W^{0}$ and $w \in W$. Then $\mathscr{F}_{i}^{M}(\cdot, z)$ and $\mathscr{F}_{i}^{W}(\cdot, z)$ are intertwining operators of type $\left(\begin{array}{c}M \\ W^{0} W\end{array}\right)$ and $\left(\begin{array}{c}W \\ W^{0} W\end{array}\right)$, respectively. Clearly, $\mathscr{F}_{i}^{M}(a, z) w+\mathscr{F}_{i}^{W}(a, z) w=$ $Y_{i}(a, z) w$. If $\mathscr{F}_{i}^{M}(\cdot, z)=0$, then $W^{0} \cdot W \subset W$ and so $V_{L}^{\tau} \cdot W=M^{0} \cdot W+W^{0} \cdot W \subset W$. This is a contradiction, since $U$ is a simple $V_{L}^{\tau}$-module. Hence $\mathscr{F}_{i}^{M}(\cdot, z) \neq 0$. Let

$$
\varphi_{i}^{W}(a, z) v=Y_{i}(a, z) v
$$

for $a \in W^{0}, v \in M$. Then $\varphi_{i}^{W}(\cdot, z)$ is a nonzero intertwining operator of type $\left(\begin{array}{c}W \\ W^{0}\end{array}\right)$ by (5-17). The space of $M^{0}$-intertwining operators $I_{M^{0}}\left(\begin{array}{c}W \\ W^{0}\end{array}\right)$ of type $\left(\begin{array}{c}W \\ W^{0}\end{array}\right)$ is of dimension one by (5-17). Similarly, $\operatorname{dim} I_{M^{0}}\left(\begin{array}{c}M \\ W^{0}{ }_{W}\end{array}\right)=\operatorname{dim} I_{M^{0}}\left(\begin{array}{c}W \\ W^{0} W\end{array}\right)=1$. Therefore, $\mathscr{F}_{2}^{M}(\cdot, z)=\lambda \mathscr{F}_{1}^{M}(\cdot, z), \mathscr{F}_{2}^{W}(\cdot, z)=\mu \mathscr{F}_{1}^{W}(\cdot, z)$, and $\mathscr{G}_{2}^{W}(\cdot, z)=\gamma \mathscr{G}_{1}^{W}(\cdot, z)$ for some $\lambda, \mu, \gamma \in \mathbb{C}$ with $\lambda \neq 0$ and $\gamma \neq 0$.

Now,

$$
\begin{aligned}
Y_{i}\left(a, z_{1}\right) Y_{i}\left(b, z_{2}\right) v & =\left(\mathscr{F}_{i}^{M}\left(a, z_{1}\right)+\mathscr{F}_{i}^{W}\left(a, z_{1}\right)\right) \mathscr{G}_{i}^{W}\left(b, z_{2}\right) v, \\
Y_{i}\left(b, z_{2}\right) Y_{i}\left(a, z_{1}\right) v & =\left(\mathscr{F}_{i}^{M}\left(b, z_{2}\right)+\mathscr{F}_{i}^{W}\left(b, z_{2}\right)\right) \mathscr{G}_{i}^{W}\left(a, z_{1}\right) v, \\
Y_{i}\left(\tilde{Y}\left(a, z_{0}\right) b, z_{2}\right) v & =Y_{i}\left(\mathscr{F}\left(a, z_{0}\right) b, z_{2}\right) v+\mathscr{G}_{i}^{W}\left(\mathscr{F}\left(a, z_{0}\right) b, z_{2}\right) v
\end{aligned}
$$


for $a, b \in W^{0}$ and $v \in M$. Taking the image of both sides of the Jacobi identity

$$
\begin{aligned}
z_{0}^{-1} \delta\left(\frac{z_{1}-z_{2}}{z_{0}}\right) Y_{i}\left(a, z_{1}\right) Y_{i}\left(b, z_{2}\right) v-z_{0}^{-1} \delta & \left(\frac{z_{2}-z_{1}}{-z_{0}}\right) Y_{i}\left(b, z_{2}\right) Y_{i}\left(a, z_{1}\right) v \\
& =z_{2}^{-1} \delta\left(\frac{z_{1}-z_{0}}{z_{2}}\right) Y_{i}\left(\tilde{Y}\left(a, z_{0}\right) b, z_{2}\right) v
\end{aligned}
$$

under the projection $p_{M}$, we obtain

$$
\begin{aligned}
z_{0}^{-1} \delta\left(\frac{z_{1}-z_{2}}{z_{0}}\right) \mathscr{F}_{i}^{M}\left(a, z_{1}\right) \varphi_{i}^{W}\left(b, z_{2}\right) v & -z_{0}^{-1} \delta\left(\frac{z_{2}-z_{1}}{-z_{0}}\right) \mathscr{F}_{i}^{M}\left(b, z_{2}\right) \varphi_{i}^{W}\left(a, z_{1}\right) v \\
& =z_{2}^{-1} \delta\left(\frac{z_{1}-z_{0}}{z_{2}}\right) Y_{i}\left(\mathscr{I}\left(a, z_{0}\right) b, z_{2}\right) v
\end{aligned}
$$

Likewise, if we take the image of both sides of (5-26) under the projection $p_{W}$, then

$$
\begin{aligned}
z_{0}^{-1} \delta\left(\frac{z_{1}-z_{2}}{z_{0}}\right) \mathscr{F}_{i}^{W}\left(a, z_{1}\right) \mathscr{G}_{i}^{W}\left(b, z_{2}\right) v & -z_{0}^{-1} \delta\left(\frac{z_{2}-z_{1}}{-z_{0}}\right) \mathscr{F}_{i}^{W}\left(b, z_{2}\right) \mathscr{G}_{i}^{W}\left(a, z_{1}\right) v \\
& =z_{2}^{-1} \delta\left(\frac{z_{1}-z_{0}}{z_{2}}\right) \mathscr{G}_{i}^{W}\left(\mathscr{F}\left(a, z_{0}\right) b, z_{2}\right) v
\end{aligned}
$$

Comparing Equation (5-28) for $i=1$ and $i=2$, we have

$$
\gamma(\mu-1) z_{2}^{-1} \delta\left(\frac{z_{1}-z_{0}}{z_{2}}\right) \varphi_{1}^{W}\left(\mathscr{g}\left(a, z_{0}\right) b, z_{2}\right) v=0,
$$

since $\mathscr{F}_{2}^{M}(\cdot, z)=\lambda \mathscr{F}_{1}^{M}(\cdot, z), \mathscr{F}_{2}^{W}(\cdot, z)=\mu \mathscr{F}_{1}^{W}(\cdot, z)$, and $\mathscr{G}_{2}^{W}(\cdot, z)=\gamma \mathscr{G}_{1}^{W}(\cdot, z)$. Now, $z_{2}^{-1} \delta\left(\frac{z_{1}-z_{0}}{z_{2}}\right)=z_{1}^{-1} \delta\left(\frac{z_{2}+z_{0}}{z_{1}}\right)$ by [Frenkel et al. 1988, Proposition 8.8.5] and so the above equation is equivalent to the following assertion.

$$
\gamma(\mu-1)\left(z_{2}+z_{0}\right)^{k} \varphi_{1}^{W}\left(\mathscr{g}\left(a, z_{0}\right) b, z_{2}\right) v=0 \text { for all } k \in \mathbb{Z} .
$$

This implies that

$$
\gamma(\mu-1) \mathscr{G}_{1}^{W}\left(\mathscr{g}\left(a, z_{0}\right) b, z_{2}\right) v=0,
$$

since $\mathscr{G}_{1}^{W}\left(\mathscr{g}\left(a, z_{0}\right) b, z_{2}\right) v \in W\left(\left(z_{0}\right)\right) \llbracket z_{2}, z_{2}^{-1} \rrbracket$. Then since $\mathscr{g}(\cdot, z)$ and $\mathscr{G}_{1}^{W}(\cdot, z)$ are nonzero, we conclude that $\mu=1$.

Next, we use Equation (5-27). Since $\Phi\left(a, z_{0}\right) b \in M^{0}\left(\left(z_{0}\right)\right)$, we have

$$
Y_{1}\left(\mathscr{T}\left(a, z_{0}\right) b, z_{2}\right) v=Y_{2}\left(\mathscr{F}\left(a, z_{0}\right) b, z_{2}\right) v
$$

by our assumption. Then it follows from (5-27) for $i=1,2$ that

$$
(\lambda \gamma-1) z_{2}^{-1} \delta\left(\frac{z_{1}-z_{0}}{z_{2}}\right) Y_{1}\left(\Phi\left(a, z_{0}\right) b, z_{2}\right) v=0 .
$$

Since $\Phi(\cdot, z) \neq 0$ and $M$ is a simple $\left(M^{0}, Y_{1}\right)$-module, a similar argument as above gives that $\lambda \gamma=1$. 
For $a \in M^{0}, b \in W^{0}, v \in M$, and $w \in W$, $Y_{i}(a+b, z)(v+w)=Y_{i}(a, z) v+Y_{i}(a, z) w+\mathscr{G}_{i}^{W}(b, z) v+\left(\mathscr{F}_{i}^{M}(b, z)+\mathscr{F}_{i}^{W}(b, z)\right) w$.

Note that $Y_{i}(a, z) v, \mathscr{F}_{i}^{M}(b, z) w \in M((z))$ and $Y_{i}(a, z) w, \mathscr{G}_{i}^{W}(b, z) v, \mathscr{F}_{i}^{W}(b, z) w \in$ $W((z))$. Define $\varphi: U \rightarrow U$ by $\varphi(u)=\lambda u$ if $u \in M$ and $\varphi(u)=u$ if $u \in W$. Since $\mu=1$ and $\lambda \gamma=1$, we can verify that

$$
Y_{2}(a+b, z) \varphi(v+w)=\varphi\left(Y_{1}(a+b, z)(v+w)\right) .
$$

Thus $\varphi$ is an isomorphism of $V_{L}^{\tau}$-modules from $\left(U, Y_{1}\right)$ onto $\left(U, Y_{2}\right)$. This completes the proof.

Remark 5.8. The proof of the above lemma is essentially the same as that of [Lam et al. 2005, Lemma C.3]. Consider the Jacobi identity for $a, b \in W^{0}$ and $w \in W$ and take the images of both sides of the identity under the projections $p_{M}$ and $p_{W}$, respectively. Then

$$
\begin{gathered}
z_{0}^{-1} \delta\left(\frac{z_{1}-z_{2}}{z_{0}}\right) \mathscr{F}_{i}^{M}\left(a, z_{1}\right) \mathscr{F}_{i}^{W}\left(b, z_{2}\right) w-z_{0}^{-1} \delta\left(\frac{z_{2}-z_{1}}{-z_{0}}\right) \mathscr{F}_{i}^{M}\left(b, z_{2}\right) \mathscr{F}_{i}^{W}\left(a, z_{1}\right) w \\
=z_{2}^{-1} \delta\left(\frac{z_{1}-z_{0}}{z_{2}}\right) \mathscr{F}_{i}^{M}\left(\mathscr{F}\left(a, z_{0}\right) b, z_{2}\right) w \\
z_{0}^{-1} \delta\left(\frac{z_{1}-z_{2}}{z_{0}}\right)\left(\mathscr{G}_{i}^{W}\left(a, z_{1}\right) \mathscr{F}_{i}^{M}\left(b, z_{2}\right)+\mathscr{F}_{i}^{W}\left(a, z_{1}\right) \mathscr{F}_{i}^{W}\left(b, z_{2}\right)\right) w \\
-z_{0}^{-1} \delta\left(\frac{z_{2}-z_{1}}{-z_{0}}\right)\left(\mathscr{G}_{i}^{W}\left(b, z_{2}\right) \mathscr{F}_{i}^{M}\left(a, z_{1}\right)+\mathscr{F}_{i}^{W}\left(b, z_{2}\right) \mathscr{F}_{i}^{W}\left(a, z_{1}\right)\right) w \\
=z_{2}^{-1} \delta\left(\frac{z_{1}-z_{0}}{z_{2}}\right)\left(Y_{i}\left(\mathscr{F}\left(a, z_{0}\right) b, z_{2}\right)+\mathscr{F}_{i}^{W}\left(\mathscr{F}\left(a, z_{0}\right) b, z_{2}\right)\right) w .
\end{gathered}
$$

Each of these two equations gives the identical equations in case of $i=1$ and $i=2$ provided that $\mu=1$ and $\lambda \gamma=1$.

Theorem 5.9. There are exactly 30 inequivalent simple $V_{L}^{\tau}$-modules. They are represented by the 30 simple $V_{L}^{\tau}$-modules listed in Lemma 3.2.

Proof. Let $U$ be a simple $V_{L}^{\tau}$-module. Then by Lemma 5.5, $U$ contains a simple $M^{0}$-submodule $M$ isomorphic to a member of $M$. Since $U$ is a simple $V_{L}^{\tau}$-module, Lemma 5.6 implies that $U=M \oplus W$ for some simple $M^{0}$-submodule $W$ isomorphic to a member of $\mathcal{W}$. In fact, the isomorphism class of $W$ is uniquely determined by $M$. By Lemma 5.7, $U$ admits a unique $V_{L}^{\tau}$-module structure. Since $M$ consists of 30 members, it follows that there are at most 30 inequivalent simple $V_{L}^{\tau}$-modules. Hence the assertion holds.

Theorem 5.10. $V_{L}^{\tau}$ is a rational vertex operator algebra. 
Proof. It is sufficient to show that every $\mathbb{N}$-graded weak $V_{L}^{\tau}$-module $U$ is a sum of simple $V_{L}^{\tau}$-modules. Since $M^{0}$ is rational, $U$ is a direct sum of simple $M^{0}$-modules. Thus by Lemma 5.2, we may assume that $U=\left(\bigoplus_{\gamma \in \Gamma} S^{\gamma}\right) \oplus\left(\bigoplus_{\lambda \in \Lambda} S^{\lambda}\right)$, where $S^{\gamma}$ is isomorphic to a member of $\mathcal{M}$ and $S^{\lambda}$ is isomorphic to a member of $\mathcal{W}$. We know that $V_{L}^{\tau} \cdot S^{\gamma}$ is a simple $V_{L}^{\tau}$-module by Lemma 5.6. Set $N=\sum_{\gamma \in \Gamma} V_{L}^{\tau} \cdot S^{\gamma}$. Since $U / N$ has no simple $M^{0}$-submodule isomorphic to a member of $\mathcal{M}$, it follows from Lemma 5.5 that $U=N$ and the proof is complete.

Corollary 5.11. The Zhu algebra $A\left(V_{L}^{\tau}\right)$ of $V_{L}^{\tau}$ is a 51 dimensional semisimple associative algebra isomorphic to a direct sum of 23 copies of the one dimensional algebra $\mathbb{C}$ and 7 copies of the algebra $\mathrm{Mat}_{2}(\mathbb{C})$ of $2 \times 2$ matrices. Moreover, $A\left(V_{L}^{\tau}\right)$ is generated by $\left[\tilde{\omega}^{1}\right],\left[\tilde{\omega}^{2}\right],[J],[K]$, and $[P]$.

Proof. Since $V_{L}^{\tau}$ is rational, $A\left(V_{L}^{\tau}\right)$ is a finite dimensional semisimple associative algebra [Dong et al. 1998a, Theorem 8.1; Zhu 1996, Theorem 2.2.3]. We know all the simple $V_{L}^{\tau}$-modules and the action of $\left[\tilde{\omega}^{1}\right],\left[\tilde{\omega}^{2}\right],[J],[K]$, and $[P]$ on their top levels in Section 4. Hence we can determine the structure of $A\left(V_{L}^{\tau}\right)$ as in the assertion.

\section{Appendix: Some fusion rules for $M(0)$}

We give a proof of the fusion rules

$$
\begin{aligned}
& W(0) \times M_{T}\left(\tau^{i}\right)(\varepsilon)=W_{T}\left(\tau^{i}\right)(\varepsilon), \\
& W(0) \times W_{T}\left(\tau^{i}\right)(\varepsilon)=M_{T}\left(\tau^{i}\right)(\varepsilon)+W_{T}\left(\tau^{i}\right)(\varepsilon),
\end{aligned}
$$

$i=1,2, \varepsilon=0,1,2$ of simple $M(0)$-modules in (3-25).

Recall that $V_{L}^{\tau} \cong M^{0} \oplus W^{0}$, where $M^{0}=M(0) \otimes M_{t}^{0}$ and $W^{0}=W(0) \otimes W_{t}^{0}$. Set $\hat{M}_{T}\left(\tau^{i}\right)(\varepsilon)=M_{T}\left(\tau^{i}\right)(\varepsilon) \otimes M_{t}^{0}$ and $\hat{W}_{T}\left(\tau^{i}\right)(\varepsilon)=W_{T}\left(\tau^{i}\right)(\varepsilon) \otimes W_{t}^{0}$, which are simple $M^{0}$-modules. Then

$$
\begin{aligned}
V_{L}^{0}(\tau)(\varepsilon) & \cong \hat{M}_{T}(\tau)(\varepsilon) \oplus \hat{W}_{T}(\tau)(\varepsilon), \\
V_{L}^{0}\left(\tau^{2}\right)(\varepsilon) & \cong \hat{M}_{T}\left(\tau^{2}\right)(\varepsilon) \oplus \hat{W}_{T}\left(\tau^{2}\right)(\varepsilon)
\end{aligned}
$$

as $M^{0}$-modules by (3-23) and (3-24). Denote by $Y_{1}(\cdot, z)\left(\right.$ resp. $\left.Y_{2}(\cdot, z)\right)$ the vertex operator of the simple $V_{L}^{\tau}$-module $V_{L}^{0}(\tau)(\varepsilon)$ (resp. $V_{L}^{0}\left(\tau^{2}\right)(\varepsilon)$ ). Let $p_{M}$ : $V_{L}^{0}(\tau)(\varepsilon) \rightarrow \hat{M}_{T}(\tau)(\varepsilon)$ and $p_{W}: V_{L}^{0}(\tau)(\varepsilon) \rightarrow \hat{W}_{T}(\tau)(\varepsilon)$ be projections. We also use the same symbol $p_{M}$ or $p_{W}$ to denote a projection from $V_{L}^{0}\left(\tau^{2}\right)(\varepsilon)$ onto $\hat{M}_{T}\left(\tau^{2}\right)(\varepsilon)$ or onto $\hat{W}_{T}\left(\tau^{2}\right)(\varepsilon)$. We fix $i=1,2$ and $\varepsilon=0,1,2$. For simplicity of notation, set $\hat{M}=\hat{M}_{T}\left(\tau^{i}\right)(\varepsilon)$ and $\hat{W}=\hat{W}_{T}\left(\tau^{i}\right)(\varepsilon)$.

Let $\mathscr{F}_{i}^{M}(a, z) w=p_{M} Y_{i}(a, z) w$ and $\mathscr{F}_{i}^{W}(a, z) w=p_{W} Y_{i}(a, z) w$ for $a \in W^{0}$ and $w \in \hat{W}$. Then $\mathscr{F}_{i}^{M}(\cdot, z)$ and $\mathscr{F}_{i}^{W}(\cdot, z)$ are intertwining operators of type $\left(\begin{array}{c}\hat{M} \\ W^{0} \hat{W}\end{array}\right)$ and 
$\left(\begin{array}{c}\hat{W} \\ W^{0} \hat{W}\end{array}\right)$, respectively. Likewise, let $\mathscr{G}_{i}^{W}(a, z) v=Y_{i}(a, z) v$ for $a \in W^{0}$ and $v \in \hat{M}$. Then $\mathscr{G}_{i}^{W}(\cdot, z)$ is an intertwining operator of type $\left(\begin{array}{c}\hat{W} \\ W^{0}\end{array} \hat{M}\right)$, since the fusion rule $W_{t}^{0} \times M_{t}^{0}=W_{t}^{0}$ of $M_{t}^{0}$-modules implies that $W^{0} \cdot \hat{M}=\operatorname{span}\left\{a_{n} \hat{M} \mid a \in W^{0}, n \in \mathbb{Z}\right\}$ is contained in $\hat{W}$. If $\varphi_{i}^{W}(\cdot, z)=0$, then $V_{L}^{\tau} \cdot \hat{M}=\left(M^{0}+W^{0}\right) \cdot \hat{M} \subset \hat{M}$. This is a contradiction, since $V_{L}^{0}(\tau)(\varepsilon)$ and $V_{L}^{0}\left(\tau^{2}\right)(\varepsilon)$ are simple $V_{L}^{\tau}$-modules. Thus $\varphi_{i}^{W}(\cdot, z) \neq 0$. Similarly, $\mathscr{F}_{i}^{M}(\cdot, z) \neq 0$. Indeed, if $\mathscr{F}_{i}^{M}(\cdot, z)=0$, then $V_{L}^{\tau} \cdot \hat{W} \subset \hat{W}$, which is a contradiction. Assume that $\mathscr{F}_{i}^{W}(\cdot, z)=0$. Then $W^{0} \cdot \hat{W} \subset \hat{M}$ and so $W^{0} \cdot\left(W^{0} \cdot \hat{W}\right) \subset \hat{W}$. However, $W^{0} \cdot\left(W^{0} \cdot \hat{W}\right) \supset\left(W^{0} \cdot W^{0}\right) \cdot \hat{W}=V_{L}^{\tau} \cdot \hat{W}$ by Lemma 2.6 and (5-18). This contradiction implies that $\mathscr{F}_{i}^{W}(\cdot, z) \neq 0$.

Restricting the three nonzero intertwining operators $\mathscr{F}_{i}^{M}(\cdot, z), \mathscr{F}_{i}^{W}(\cdot, z)$, and $\varphi_{i}^{W}(\cdot, z)$ to the first component of each of the tensor products $W^{0}=W(0) \otimes W_{t}^{0}$, $\hat{M}=M_{T}\left(\tau^{i}\right)(\varepsilon) \otimes M_{t}^{0}$, and $\hat{W}=W_{T}\left(\tau^{i}\right)(\varepsilon) \otimes W_{t}^{0}$, we obtain nonzero intertwining operators of type

$$
\left(\begin{array}{c}
M_{T}\left(\tau^{i}\right)(\varepsilon) \\
W(0) W_{T}\left(\tau^{i}\right)(\varepsilon)
\end{array}\right), \quad\left(\begin{array}{c}
W_{T}\left(\tau^{i}\right)(\varepsilon) \\
W(0) W_{T}\left(\tau^{i}\right)(\varepsilon)
\end{array}\right) \quad\left(\begin{array}{c}
W_{T}\left(\tau^{i}\right)(\varepsilon) \\
W(0) M_{T}\left(\tau^{i}\right)(\varepsilon)
\end{array}\right)
$$

for $M(0)$-modules, respectively.

Let $N^{2}$ be one of $M_{T}\left(\tau^{i}\right)(\varepsilon), W_{T}\left(\tau^{i}\right)(\varepsilon), i=1,2, \varepsilon=0,1,2$ and let $N^{3}$ be any of the 20 simple $M(0)$-modules. Then the top level $N_{(0)}^{j}$ of $N^{j}$ is of dimension one. By [Dong et al. 2004], the Zhu algebra $A(M(0))$ of $M(0)$ is generated by [ $\left.\tilde{\omega}^{1}\right]$ and $[J]$. Moreover, we know the action of $o\left(\tilde{\omega}^{1}\right)$ and $o(J)$ on $N_{(0)}^{j}$. Thus, by an argument as in [Tanabe 2005, pp. 192-193], we can calculate that the dimension of

$$
\operatorname{Hom}_{A(M(0))}\left(A(W(0)) \otimes_{A(M(0))} N_{(0)}^{2}, N_{(0)}^{3}\right)
$$

is at most one and it is equal to one if and only if the pair $\left(N^{2}, N^{3}\right)$ is one of

$$
\left(M_{T}\left(\tau^{i}\right)(\varepsilon), W_{T}\left(\tau^{i}\right)(\varepsilon)\right), \quad\left(W_{T}\left(\tau^{i}\right)(\varepsilon), M_{T}\left(\tau^{i}\right)(\varepsilon)\right), \quad\left(W_{T}\left(\tau^{i}\right)(\varepsilon), W_{T}\left(\tau^{i}\right)(\varepsilon)\right)
$$

for $i=1,2, \varepsilon=0,1,2$. Note that $W(0)$ was denoted by $W_{k}^{0(0)}$ in [Tanabe 2005]. Now, the desired fusion rules are obtained by [Li 1999a, Proposition 2.10 and Corollary 2.13].

\section{References}

[Abe and Dong 2004] T. Abe and C. Dong, "Classification of irreducible modules for the vertex operator algebra $V_{L}^{+}$: general case”, J. Algebra 273:2 (2004), 657-685. MR 2005c:17040 Zbl 1051.17015

[Abe et al. 2004] T. Abe, G. Buhl, and C. Dong, "Rationality, regularity, and $C_{2}$-cofiniteness", Trans. Amer. Math. Soc. 356:8 (2004), 3391-3402. MR 2005c:17041 Zbl 1070.17011

[Abe et al. 2005] T. Abe, C. Dong, and H. Li, "Fusion rules for the vertex operator algebra M(1) and $V_{L}^{+}$, Comm. Math. Phys. 253:1 (2005), 171-219. MR 2005i:17033 Zbl pre05076366 
[Bouwknegt et al. 1996] P. Bouwknegt, J. McCarthy, and K. Pilch, The $W_{3}$ algebra: Modules, semi-infinite cohomology and BV algebras, Lecture Notes in Physics M42, Springer, Berlin, 1996. MR 97m:17029

[Buhl 2002] G. Buhl, “A spanning set for VOA modules”, J. Algebra 254:1 (2002), 125-151. MR 2003m:17022 Zbl 1037.17032

[Dong 1993] C. Dong, "Vertex algebras associated with even lattices", J. Algebra 161:1 (1993), 245-265. MR 94j:17023 Zbl 0807.17022

[Dong and Lepowsky 1996] C. Dong and J. Lepowsky, "The algebraic structure of relative twisted vertex operators", J. Pure Appl. Algebra 110:3 (1996), 259-295. MR 98e:17036 Zbl 0862.17021

[Dong and Mason 1997] C. Dong and G. Mason, "On quantum Galois theory", Duke Math. J. 86:2 (1997), 305-321. MR 97k:17042 Zbl 0890.17031

[Dong and Yamskulna 2002] C. Dong and G. Yamskulna, "Vertex operator algebras, generalized doubles and dual pairs", Math. Z. 241:2 (2002), 397-423. MR 2003j:17038 Zbl 1073.17009

[Dong et al. 1997] C. Dong, H. Li, and G. Mason, "Regularity of rational vertex operator algebras", Adv. Math. 132:1 (1997), 148-166. MR 98m:17037 Zbl 0902.17014

[Dong et al. 1998a] C. Dong, H. Li, and G. Mason, "Twisted representations of vertex operator algebras”, Math. Ann. 310:3 (1998), 571-600. MR 99d:17030 Zbl 0890.17029

[Dong et al. 1998b] C. Dong, H. Li, G. Mason, and S. P. Norton, "Associative subalgebras of the Griess algebra and related topics", pp. 27-42 in The Monster and Lie algebras (Columbus, OH, 1996), edited by J. Ferrar and K. Harada, Ohio State Univ. Math. Res. Inst. Publ. 7, de Gruyter, Berlin, 1998. MR 99k:17048 Zbl 0946.17011

[Dong et al. 2000] C. Dong, H. Li, and G. Mason, "Modular-invariance of trace functions in orbifold theory and generalized Moonshine", Comm. Math. Phys. 214:1 (2000), 1-56. MR 2001k:17043 Zbl 1061.17025

[Dong et al. 2004] C. Dong, C. H. Lam, K. Tanabe, H. Yamada, and K. Yokoyama, " $\mathbb{Z}_{3}$ symmetry and $W_{3}$ algebra in lattice vertex operator algebras", Pacific J. Math. 215:2 (2004), 245-296. MR 2005c:17045 Zbl 1055.17013

[Frenkel et al. 1988] I. Frenkel, J. Lepowsky, and A. Meurman, Vertex operator algebras and the Monster, Pure and Applied Mathematics 134, Academic Press, Boston, 1988. MR 90h:17026 Zbl 0674.17001

[Frenkel et al. 1993] I. B. Frenkel, Y.-Z. Huang, and J. Lepowsky, On axiomatic approaches to vertex operator algebras and modules, Mem. Amer. Math. Soc. 494, Amer. Math. Soc., Providence, RI, 1993. MR 94a:17007 Zbl 0789.17022

[Gaberdiel and Neitzke 2003] M. R. Gaberdiel and A. Neitzke, "Rationality, quasirationality and finite W-algebras”, Comm. Math. Phys. 238:1-2 (2003), 305-331. MR 2004g:81084 Zbl 1042.17025 [Kitazume et al. 2000a] M. Kitazume, C. H. Lam, and H. Yamada, "Decomposition of the Moonshine vertex operator algebra as Virasoro modules”, J. Algebra 226:2 (2000), 893-919. MR 2001f: 17055 Zbl 0986.17010

[Kitazume et al. 2000b] M. Kitazume, M. Miyamoto, and H. Yamada, "Ternary codes and vertex operator algebras”, J. Algebra 223:2 (2000), 379-395. MR 2000m:17032 Zbl 0977.17026

[Kitazume et al. 2003] M. Kitazume, C. H. Lam, and H. Yamada, "3-state Potts model, Moonshine vertex operator algebra, and 3A-elements of the Monster group", Int. Math. Res. Not. 2003:23 (2003), 1269-1303. MR 2004b:17051 Zbl 1037.17034

[Lam and Yamada 2000] C. H. Lam and H. Yamada, " $Z_{2} \times Z_{2}$ codes and vertex operator algebras", J. Algebra 224:2 (2000), 268-291. MR 2001d:17029 Zbl 1013.17025

[Lam et al. 2005] C. H. Lam, H. Yamada, and H. Yamauchi, "McKay's observation and vertex operator algebras generated by two conformal vectors of central charge 1/2", IMRP Int. Math. Res. Pap. 2005:3 (2005), 117-181. MR 2006h:17034 Zbl 1082.17015 
[Lepowsky 1985] J. Lepowsky, "Calculus of twisted vertex operators", Proc. Nat. Acad. Sci. U.S.A. 82:24 (1985), 8295-8299. MR 88f:17030 Zbl 0579.17010

[Lepowsky and Li 2004] J. Lepowsky and $\mathrm{H}$. Li, Introduction to vertex operator algebras and their representations, Progress in Mathematics 227, Birkhäuser, Boston, 2004. MR 2004k:17050 Zbl 1055.17001

[Li 1999a] H. Li, "Determining fusion rules by $A(V)$-modules and bimodules", J. Algebra 212:2 (1999), 515-556. MR 2000d:17032 Zbl 0977.17027

[Li 1999b] H. Li, "Some finiteness properties of regular vertex operator algebras", J. Algebra 212:2 (1999), 495-514. MR 2000c:17044 Zbl 0953.17017

[Li 2001] H. Li, “The regular representation, Zhu's A(V)-theory, and induced modules”, J. Algebra 238:1 (2001), 159-193. MR 2003f:17039 Zbl 1060.17015

[Miyamoto 2001] M. Miyamoto, "3-state Potts model and automorphisms of vertex operator algebras of order 3”, J. Algebra 239:1 (2001), 56-76. MR 2002c:17042 Zbl 1022.17020

[Miyamoto and Tanabe 2004] M. Miyamoto and K. Tanabe, "Uniform product of $A_{g, n}(V)$ for an orbifold model $V$ and $G$-twisted Zhu algebra", J. Algebra 274:1 (2004), 80-96. MR 2005d:17037 Zbl 1046.17009

[Nagatomo and Tsuchiya 2005] K. Nagatomo and A. Tsuchiya, "Conformal field theories associated to regular chiral vertex operator algebras. I. Theories over the projective line", Duke Math. J. 128:3 (2005), 393-471. MR 2006e:81258 Zbl 1074.81065

[Tanabe 2005] K. Tanabe, "On intertwining operators and finite automorphism groups of vertex operator algebras”, J. Algebra 287:1 (2005), 174-198. MR 2006e:17041 Zbl 02173135

[Wang 1993] W. Wang, "Rationality of Virasoro vertex operator algebras", Internat. Math. Res. Notices 1993:7 (1993), 197-211. MR 94i:17034 Zbl 0791.17029

[Yamauchi 2004] H. Yamauchi, "Modularity on vertex operator algebras arising from semisimple primary vectors", Internat. J. Math. 15:1 (2004), 87-109. MR 2005i:17036 Zbl 1052.17018

[Yamskulna 2004] G. Yamskulna, " $C_{2}$-cofiniteness of the vertex operator algebra $V_{L}^{+}$when $L$ is a rank one lattice", Comm. Algebra 32:3 (2004), 927-954. MR 2005h:17053 Zbl 1093.17008

[Zhu 1996] Y. Zhu, "Modular invariance of characters of vertex operator algebras", J. Amer. Math. Soc. 9:1 (1996), 237-302. MR 96c:17042 Zbl 0854.17034

Received August 11, 2005. Revised January 11, 2006.

\author{
Kenichiro TANABE \\ DEPARTMENT OF MATHEMATICS \\ HOKKAIDO UNIVERSITY \\ KITA 10, NiSHI 8, KITA-KU \\ SAPPORO, HOKKAIDO 060-0810 \\ JAPAN \\ ktanabe@math.sci.hokudai.ac.jp \\ HIROMICHI YAMADA \\ DEPARTMENT OF MATHEMATICS \\ HiTOTSUBASHI UNIVERSITY \\ NAKA 2-1, KUNITACHI \\ TOKYO 186-8601 \\ JAPAN \\ yamada@math.hit-u.ac.jp
}

